Journal of Global Economic Analysis, Volume 2 (2017), No. 1, pp. 215-324.

\title{
Current Account Balances, Exchange Rates, and Fundamental Properties of Walrasian CGE World Models: A Pedagogical Exposition
}

\author{
BY ANDRÉ LEMELINa
}

This paper addresses theoretical aspects of global multinational trade models of the computable general equilibrium (CGE) type. We define and discuss the concepts of model homogeneity, model closure rules, and consistency in calibration. We examine and illustrate these issues using a highly simplified skeleton model derived from the PEP-w-1 CGE world model, to represent the essential structure of world trade models. Model closure issues, including how to correctly fix current account balances, are scrutinized. We also consider the role of nominal exchange rates in Walrasian "real" CGE models (without money), which can be, and often are written without exchange rates. But when exchange rates are present, we show that a model can be solved equivalently by exogenously fixing either exchange rates (FE) or regional price indexes (FP), and we weigh the advantages of either closure for economic interpretation of simulation results. The model is implemented in GAMS and is made available to readers as a supplementary download.

JEL codes: C68, D58, F47

Keywords: Computable general equilibrium models (CGE); Global trade models; CGE model closures; Current account balance; Exchange rate.

\section{Introduction}

There is an abundant literature on macro-economic closures for single country, open-economy models (Decaluwé et al., 1988; Delpiazzo, 2010 contains a recent comprehensive review; Dewatripont and Michel, 1987; Rattsø, 1982; Sen, 1963). In the present paper, however, we are not concerned with closures in single-economy models, but rather with issues that arise in the context of global multinational models, typically, trade models. In addition to model closures, we explore a few

\footnotetext{
a Urbanization and Culture Society, Institut National de la Recherche Scientifique (INRSUCS), 385 rue Sherbrooke est, Montréal, QC, Canada H2X 1E3 (andre_lemelin@ucs.inrs.ca).
} 
related issues that are rarely dealt with explicitly in CGE multinational modeling: model homogeneity, the role of exchange rates, calibration neutrality and consistency, and testing for calibration consistency and model homogeneity.

Indeed, there seems to be a void in the literature regarding these issues, each of which will be addressed in turn.

Closures: Global models, as opposed to single-country, open-economy models or multiregional non-global models, are "closed" systems, in that there is no "Rest of the world". It follows that the worldwide sum of current account balances, when expressed in a common currency, must be zero. This rules out the unconstrained flexible current account closure sometimes used in non-global models. As a matter of fact, exogenous trade balances (which ensure the zero-sum constraint) are a common form of closure in global models, but the constraint is seldom explicitly stated or its form motivated. ${ }^{1}$ We also extend the formal discussion of closures to systematically examine other possibilities, only to conclude that they are not feasible. This underlines the fact that selecting closure rules cannot be reduced to the simple counting of equations and variables.

Exchange rates: Another feature of global models is that the economic reality they represent involves multiple currencies. Data sources such as the GTAP database convert all monetary values to a common currency, and many global models are implemented without exchange rates, for example GTAP (Hertel 1997), or MIRAGE (Bchir et al., 2002a, 2002b; Decreux and Valin, 2007). In most Walrasian CGE world models that do have exchange rates, their nominal values are fixed (one exception is the Globe model: McDonald and Thierfelder, 2016). Here we propose endogenous nominal exchange rates, with fixed regional numéraires, as a model design which facilitates interpretation of results.

Calibration: Calibration procedures, including the implications of price $\times$ volume factoring, are generally not discussed. ${ }^{2}$ Here, the concepts of homogeneity, closure and calibration neutrality and consistency are defined, and then related to practical issues of model specification, in a single integrated exposition. This third set of issues relates to any CGE model, not only global ones.

To examine and illustrate the issues we tackle, we develop a highly simplified skeleton model derived from the PEP-w-1 worldwide CGE model (Lemelin,

\footnotetext{
${ }^{1}$ The topic of endogenous current account balances, often discussed under the heading of "international capital mobility", is not addressed in this paper. Indeed, capital is internationally mobile and current account balances are endogenous in several global CGE dynamic models: the current version of GTAP and GTAP-Dyn (Ianchovichina and McDougall, 2001; Ianchovichina et al., 2000); the G-Cubed model (McKibbin and Stoeckel, 2009; McKibbin and Wilcoxen, 1999); the DART model (Hübler, 2011; Springer, 2003); the PEP-w-t-fin model (Lemelin, Robichaud, and Decaluwé, 2013, 2014).

2 McDonald and Thierfelder (2016, p.19) state their normalization rule explicitly, but do not discuss it.
} 
Robichaud, Decaluwé \& Maisonnave, 2013), but which represents the essential structure of several world trade models. It should be mentioned that our skeleton model has been elaborated using a SAM (Social Accounting Matrx)-based approach and has been implemented in GAMS. It follows that some technical points may not be relevant to other approaches (such as models implemented in GEMPACK), but the principles discussed are generally applicable to CGE modeling.

In Section 2, we present the basic concepts: model homogeneity, model closure, and calibration consistency. The theoretical model is presented in Section 3 (Model 1). In Section 4, the model is simplified, and redundant equations are identified and deleted (Model 2). Closure rules are first discussed in reference to Model 2 (Section 5). Next (Section 6), the role of exchange rates is clarified; the model is rewritten in terms regional currencies, and nominal exchange rates are introduced (Model 3). Closure rules are revisited, detailing the choice between fixed exchange rates (FE) or fixed regional price (FP) closures; and calibration consistency is discussed. A brief conclusion wraps up the paper.

The implementation of Model 3 in GAMS is presented in Appendix K, and all of the GAMS programs and files are provided with this paper as supplementary online material. Readers are strongly encouraged to use the GAMS programs to test some of the ideas raised in the paper and thus gain a more hands-on understanding.

\section{Concepts}

\subsection{Model homogeneity and Walras' Law}

Model homogeneity derives from microeconomic theory. The theory states that supply and demand functions derived from optimizing are homogenous of degree zero in prices and income (or more generally prices and nominal values). Formally, a CGE model is a set of simultaneous equations relating variables, some of which are endogenous (determined within the model), the rest being exogenous. The core of a CGE model consists of equations representing consumer and producer optimizing behavior, and market equilibrium. A CGE model solution is a Walrasian competitive general equilibrium: all optimizing economic agents meet their (first-order) optimality conditions, subject to their budget constraints, and all markets are in equilibrium. Without money, the set of equations which constitute the model is homogenous of degree zero in prices.

To formalize the definition of homogeneity and generalize it a little, let us distinguish the three types of variables a model may contain: volume variables, price variables, and nominal variables. Some nominal variables are the product of a volume and a price, but others cannot be factored into volume and price. They just represent payments made from one agent to another, such as transfers. With that distinction in mind, a model solution may be characterized as a triplet of 
vectors $\{\mathbf{q}, \mathbf{p}, \mathbf{n}\}$, containing volume $(\mathbf{q})$, price $(\mathbf{p})$, and nominal (n) variables. In a homogenous model, if $\{\mathbf{q}, \mathbf{p}, \mathbf{n}\}$ is a solution to the model, then $\{\mathbf{q}, \lambda \mathbf{p}, \lambda \mathbf{n}\}$ is also a solution, for any $\lambda>0$. In other words, multiplying all prices and nominal values by a constant doesn't disturb the equilibrium, because it leaves relative prices unchanged. This is the principal implication of homogeneity: only relative prices matter. Prices are determined only up to a factor of proportionality and their absolute level is indeterminate.

The absolute level of prices is indeterminate because model homogeneity implies that one equation is redundant: there is one more price variable than the number of independent market equilibrium equations. This is generally referred to as Walras' Law (Léon Walras, 1834-1910). Consider an economy where producers maximize their profits subject to their production function, and consumers own all factors of production, receive all factor income, and maximize their utility subject to their budget constraint. ${ }^{3}$ For every good (factor or commodity), excess demand is defined as the sum of demands by all agents, minus the sum of supplies by all agents, for some price vector.Denote the vector of excess demands as $\xi(\mathbf{p})$. In equilibrium, demand equals supply, and $\xi\left(\mathbf{p}^{*}\right)=0$, where $\mathbf{p}^{*}$ is the equilibrium price vector. Walras' Law states that for any $\mathbf{p}$ (equilibrium or not), the total value of excess demands is zero: $\mathbf{p}^{\prime} \xi(\mathbf{p})=0$. This is a straightforward consequence of respecting budget constraints: since $\mathbf{p}^{\prime} \xi(\mathbf{p})$ is the sum of all expenditures and incomes of all agents, if all budget constraints are satisfied, then that sum must be zero. A corollary of Walras' Law is that, if all markets but one are in equilibrium for some price vector, then the remaining market must also be in equilibrium, because the value of excess demand on that market cannot be different from zero. It follows that in a model with $N$ markets, there are only $N-1$ independent market equilibrium equations, and the $N^{\text {th }}$ is redundant.

That leaves one degree of freedom, and the model is completed by exogenously fixing the price of one good, which plays the role of numéraire. The value assigned to the price of the numéraire determines the level of prices, and all other prices and nominal values in the model are expressed in terms of the price of the numéraire. ${ }^{4}$

3 This implies that all profits are distributed to consumers. Walras' Law can be demonstrated in a less restrictive setting, but our objective here is to put forth the principle behind Walras' Law, and so we keep its exposition as simple as possible.

${ }^{4}$ Strictly speaking, the word numéraire designates the commodity relative to the price of which the prices of all other commodities are expressed. But for convenience, we take the shortcut of using the word "numéraire" to designate the price of the numéraire commodity. This allows us to say, for example, that all prices are expressed in terms of the numéraire 
Homogeneity has two implications regarding the choice of a numéraire and of its value. First, although it may be convenient to set the numéraire at 1 , the value assigned to the numéraire is arbitrary. That is quite obvious from the definition of homogeneity: if $\{\mathbf{q}, \mathbf{p}, \mathbf{n}\}$ is a solution to the model when the numéraire is set at 1 , then $\{\mathbf{q}, \lambda \mathbf{p}, \lambda \mathbf{n}\}$ is also a solution, when the numéraire is set at any $\lambda>0$. A second implication is that the choice of numéraire is arbitrary. Suppose that commodity $i$ is chosen as the numéraire, with its price $p_{i}$ set at $p_{i}^{0}$; then changing the numéraire for commodity $j$ and setting its price at $p_{j}^{1}$ is equivalent to changing from solution $\{\mathbf{q}, \mathbf{p}, \mathbf{n}\}$ to $\{\mathbf{q}, \lambda \mathbf{p}, \lambda \mathbf{n}\}$ with $\lambda=p_{j}^{1} / p_{j}^{0}$, where $p_{j}^{0}$ is the value of price $j$ when the numéraire is commodity $i$.

In other words, if a model is truly homogenous, the solution values of real (volume) variables and all price and nominal value ratios are supposed to be: independent of which commodity is taken as the numéraire; independent of which region is taken as the reference region when the numéraire is a regional commodity (a particular case of the preceding); and independent of the particular value given the price of the numéraire, whatever commodity plays that role.

Of course, not all CGE models are "purely" Walrasian. But most non-monetary CGE models nevertheless retain the property of model homogeneity. In any case, if a model is not homogeneous, it should be by purpose, not by accident or by mistake. So it is useful to check for model homogeneity.

It is easy enough to check simple models for homogeneity, but it may be tricky with complex models. With simple models, one can check for homogeneity analytically, by examining the equations. Homogeneity can also be verified numerically, by transforming a $\{\mathbf{q}, \mathbf{p}, \mathbf{n}\}$ simulation solution into a $\{\mathbf{q}, \lambda \mathbf{p}, \lambda \mathbf{n}\}$ solution, and then verifying whether the model equations are satisfied. Another approach, somewhat more demanding, is to compare simulation solutions obtained with different values of the numéraire or with different numéraires and verify that the alternate solutions $\{\mathbf{q}, \mathbf{p}, \mathbf{n}\}$ and $\left\{\mathbf{q}^{\prime}, \mathbf{p}^{\prime}, \mathbf{n}^{\prime}\right\}$ satisfy the relationship $\left\{\mathbf{q}^{\prime}, \mathbf{p}^{\prime}, \mathbf{n}^{\prime}\right\}=\{\mathbf{q}, \lambda \mathbf{p}, \lambda \mathbf{n}\}$ for some $\lambda>0$.

The latter approach is more demanding, because it requires that the model equations be written in such a way as to adjust to an arbitrary value of an arbitrary choice of numéraire (this is illustrated in the homogeneity tests outlined in Section 6.3). ${ }^{5}$ Moreover, the model closure must be formulated with care to avoid the many

- much more concise and comprehensible than the first sentence of this footnote! In addition, it should be mentioned that in some models, prices are expressed relative to some global price index, in which case the numeraire good is an aggregate.

${ }^{5}$ I understand that in GEMPACK, model homogeneity is automatically checked by shocking the numéraire. 
pitfalls of inadvertantly de-homogenizing the model. Regarding our skeleton model, we shall see, in particular, that, under fixed exogenous current account balance closures, the way current account balances are fixed is critical (Section 4.2).

\subsection{Model closure}

With a CGE model, as with any system of simultaneous equations, the number of independent equations must be equal to the number of endogenous variables for the model to have a solution (the model must be square). ${ }^{6}$ If there are more equations than variables, the model is overdetermined; if there are fewer equations than variables, the model is underdetermined. The issue of model closure concerns the theoretical foundations and meaning of the choices made by the modeler to make the number of equations and the number of variables equal.

In a way, taking into account Walras' Law to discard a redundant equation, and defining a numéraire to make the model square, can be viewed as one aspect of model closure. But the issue of model closure is broader and more substantive.

The discussion of macroclosures in single-economy models was initiated by Sen (1963) in relation to the debate on income distribution. Essentially, Sen showed that competing views of the economy could be characterized by the choice of which equation to eliminate in an overdetermined model (this is nicely summarized in Rattsø, 1983). In contrast, contemporary CGE models are usually underdetermined, left open to different views of the economy. It is up to the model user to choose which equation or constraint to add to the model in order to "close" it and to make it "square", with as many equations as there are variables.7

Here we are concerned with multinational (or multiregional) world models, and we will examine model closures that take the simple form of fixing one or more variables exogenously. Specifically, we consider:

1) How many variables must be exogenously fixed?

2) Which variables can be sensibly designated as exogenous to close the model?

3) Does the choice of exogenous variables always matter? Can the same model closure be implemented by fixing alternative sets of variables? Put otherwise, are there model closures which are different in their implementation, but are mathematically and economically equivalent?

Underlying question 1 is the matter of redundant equations. The number of variables to fix exogenously is equal to the number of degrees of freedom in the model, given by the difference between the number of endogenous variables and the number of independent equations. To accurately count the number of

\footnotetext{
${ }^{6}$ It would be tempting to add "unique". But the possibility of multiple solutions cannot always be ruled out in models that are constrained nonlinear systems (CNS).

${ }^{7}$ For example, see "Macroeconomic balances", pp. 14-17 in Lofgren et al. (2002).
} 
independent equations, one must be able to identify redundant equations in the model. This is of practical importance when the model is submitted to a GAMS solver as a CNS (constrained nonlinear system) class model, because GAMS will reject the model as not square if it contains redundant equations, even if, mathematically, redundant equations do no harm.

\subsection{Calibration neutrality and consistency}

To implement a CGE model, values must be assigned to its parameters and benchmark variables. This process is called model parametrization and the usual practice in CGE modeling is to proceed in two steps: (1) assign values to a set of so-called "free" parameters, and (2) calibrate the model. The distinction between free and calibrated parameters is somewhat arbitrary, but it is customary to treat elasticities in behavioral functions as free parameters, for which modelers use econometric estimates, or else borrow estimates from the literature. Model calibration on the other hand is the process of assigning values to parameters and benchmark variables, given the values of free parameters, from the information contained in the underlying data base (often organized in the form of a social accounting matrix - SAM), combined with the restrictions imposed by the theoretical specification of the model (model equations).

Price $\times$ volume factoring: Variable benchmark values in particular are routinely calibrated from SAMs. SAM entries are transaction flows. Part of the calibration procedure consists in a factoring of SAM transaction flows into price $\times$ volume products. But SAM transaction flows generally represent composite aggregates for which there is no clear physical unit of measurement. Even when the volume can be measured unambiguously, factoring depends on the choice of unit of measurement, which is arbitrary: 454 grams of something at 14 a gram is the same as one pound at $\$ 4.54$ per pound. It follows that the price $\times$ volume factoring is arbitrary, constrained only by the value of their product. However, although the factoring of SAM transaction flows is arbitrary, there are consistency requirements to satisfy: we return to these shortly.

But first, let us briefly recall an implication of the arbitrariness of price $\times$ volume factoring that is occasionally neglected in the interpretation of CGE simulation results, namely that prices and volumes must be viewed as indices: price or volume levels are meaningless; only proportional changes are meaningful. ${ }^{8}$ When, in a simulation solution, a transaction flow increases or decreases relative to its benchmark value, the CGE model provides a decomposition of the change into price

8 Of course, if complementary information makes it possible to convert SAM flows into proper quantities, then the volume indices can be translated to quantities. For example, the volume of full-time equivalent employment, when it is known, provides a metric to convert the SAM flow of labor income into the corresponding number of jobs. 
variation and volume variation. This is exactly the kind of decomposition that is needed to correctly interpret, say, an increase in consumption expenditures when the consumer price index changes.

Neutrality of the identification constraints: Once the so-called "free" parameters (elasticities and the like) have been specified, the number of unknowns (parameters and benchmark variables) usually remains greater than the number of constraints (SAM values and model equations). Therefore, to solve the calibration problem, it is necessary to impose additional constraints, which we shall call "identification constraints". Identification constraints often consist in fixing the level of a set of variables which are defined only up to a factor of proportionality (like in price $\times$ volume factoring). There is in general no unique set of identification constraints that will complete the calibration procedure, but such constraints, given that they are based neither on observation (SAM flows), nor on theory (model equations), should be non-restrictive, or "neutral". By that, we mean that they should not affect model results. Formally, we define neutrality as follows. Given a consistent calibration procedure, a set of identification constraints is said to be neutral if it results in a model for which the relative variation of a variable between any simulation solution and its benchmark is the same as with any other set of neutral identification constraints. ${ }^{9}$

A concrete example of neutrality is given in subsection 6.2 with respect to the arbitrariness of price $\times$ volume factoring. We show that the volume of a constant elasticity of substitution (CES) or constant elasticity of transformation (CET) aggregate may be benchmarked as the sum of its components without implicitly imposing perfect substitutability, or alternatively, that the price of the aggregate may be arbitrarily set at 1 without imposing price equality.

Consistency of the calibration procedure: The calibration procedure solves what has in effect become a simultaneous equations problem, as defined by the values of free parameters, the model data base (SAM), the model specification (equations), and the identification constraints. But rather than being shifted to a solver program, the calibration problem is generally solved through a sequence of statements assigning values to the unknown parameters and benchmark variables. This is equivalent to solving the system of equations after having triangularized

\footnotetext{
${ }^{9}$ There is the appearance of a circular problem here. The model cannot be solved if it is not calibrated, and it cannot be calibrated without some set of identification constraints. So checking numerically whether simulation results are the same with or without a given set of identification constraints actually means comparing simulation results obtained using two different sets of identification constraints. Unless one of the two has been demonstrated to be neutral, the comparison is not logically conclusive. However, in this modeler's experience, there usually exists a set of identification constraints that can be shown mathematically to be neutral.
} 
the problem. So the art of calibration is very much the art of correctly sequencing the assignments.

What then is "calibration consistency"? Formally, calibration consistency could be defined as the requirement that the calibration procedure be truly equivalent to solving the (correctly) triangularized calibration problem. Practically speaking, calibration inconsistency may result from errors in the sequencing of assignments or in formulations that fail to preserve the neutrality of some identification constraint.

Test of neutrality and consistency: Two models are equivalent if the relative variation of all variables between any simulation solution and the benchmark is the same in both. To test a calibration procedure against another is to determine whether the models that result from the two procedures are equivalent or not. Mathematically, let $P_{i, k}^{C, S}$ be the value of price variable $i$, in solution $k$ of the model calibrated using a calibration procedure $C$ and a set $S$ of identification constraints; subscript $k$ is equal to 0 for the benchmark, and to 1 for the simulation. Also let $Q_{j, k}^{C, S}$ be the value of volume variable $j$ in solution $k$ of the model calibrated using $C$ and $S$. Sets $S$ may differ in the choice of variables or in the values assigned or both. Then the resulting models are equivalent if

$$
\begin{gathered}
\frac{P_{i, 1}^{C 1, S 1} / P_{i, 0}^{C 1, S 1}}{P_{h, 1}^{C 1, S 1} / P_{h, 0}^{C 1, S 1}}=\frac{P_{i, 1}^{C 2, S 2} / P_{i, 0}^{C 2, S 2}}{P_{h, 1}^{C 2, S 2} / P_{h, 0}^{C 2, S 2}} \\
Q_{j, 1}^{C 1, S 1} / Q_{j, 0}^{C 1, S 1}=Q_{j, 1}^{C 2, S 2} / Q_{j, 0}^{C 2, S 2}
\end{gathered}
$$

for any pair $C 1, C 2$ of calibration procedures, and pair $S 1, S 2$ of sets of identification calibration constraints. The first condition states that variations in the price of any good $i$ relative to any other $h$ must be the same in both cases; the second condition says that proportional volume variations must be equal.

If these two conditions are not realized, it may be for one of two reasons. First, one of the pair of sets of constraints $S 1$ and $S 2$, or both, may be restrictive (nonneutral). In that case, the problem is with the identification constraints, not with the calibration procedure. And either the identification constraints must be replaced with a set of neutral constraints, or the model specification should be extended to include one or more of these non-neutral constraints. As an example of the latter situation, the assignment of values to elasticity parameters is not neutral; for that reason, elasticities are treated as "free" parameters, not as identification constraints, and they are part of the model specification.

The second reason for which conditions (1) and (2) may be violated is that the calibration procedure is not consistent. In particular, the way in which the 
constraints are implemented may be inconsistent in the sense that the calibration procedure is compatible only with one particular set of identification constraints. An example is given in subsection 6.3.3, where a calibration shortcut which sets a given price at 1 is valid only if other previously determined prices are also set at 1 , while their value should be arbitrary.

To summarize, model parameters and benchmark values cannot be calibrated from SAM values and model equations alone, even after assigning values to the free parameters: the solution of the calibration problem remains undetermined without additional "identifying" constraints. However, these identifying constraints are not totally arbitrary: they must be neutral and applied consistently. Moreover, the calibration procedure must be a correct implementation of the solution to the triangularized calibration problem.

\section{Skeleton model: Model 1}

We now define the theoretical model which will serve as our departure point to explore the issues raised in the introduction.

\subsection{Model description}

There are $N$ regions. There is a single regional agent in each region, and there are no taxes. All prices and nominal values are expressed in terms of the international currency.

There is a single good, and production factors are fixed. With full employment, this implies that output is fixed in each region $z$, and that the regional agent's income is equal to the value of production.

The model is static, so we can define "consumption" to include both current consumption and investment. It follows that domestic savings, the difference between income and "consumption" (which includes savings), are equal to the current account balance (CAB). ${ }^{10}$

The model retains the Armington hypothesis regarding the distribution of demand between imports and the domestically produced good, and for imports, between source regions, using CES aggregator functions. Similarly, production is allocated between domestic sales and exports, and for exports, between regions of destination, according to CET functions.

This model is close to a Walrasian pure exchange economy. It is illustrated in Figure 1 for the case of two regions. But in what follows, we deal with an $N$-region model.

\footnotetext{
${ }^{10}$ In other words, in this skeleton model, the definition of domestic savings is much narrower than normally: here, it is the surplus of income over domestic absorption, whereas it is normally the surplus of income over current private and public consumption.
} 


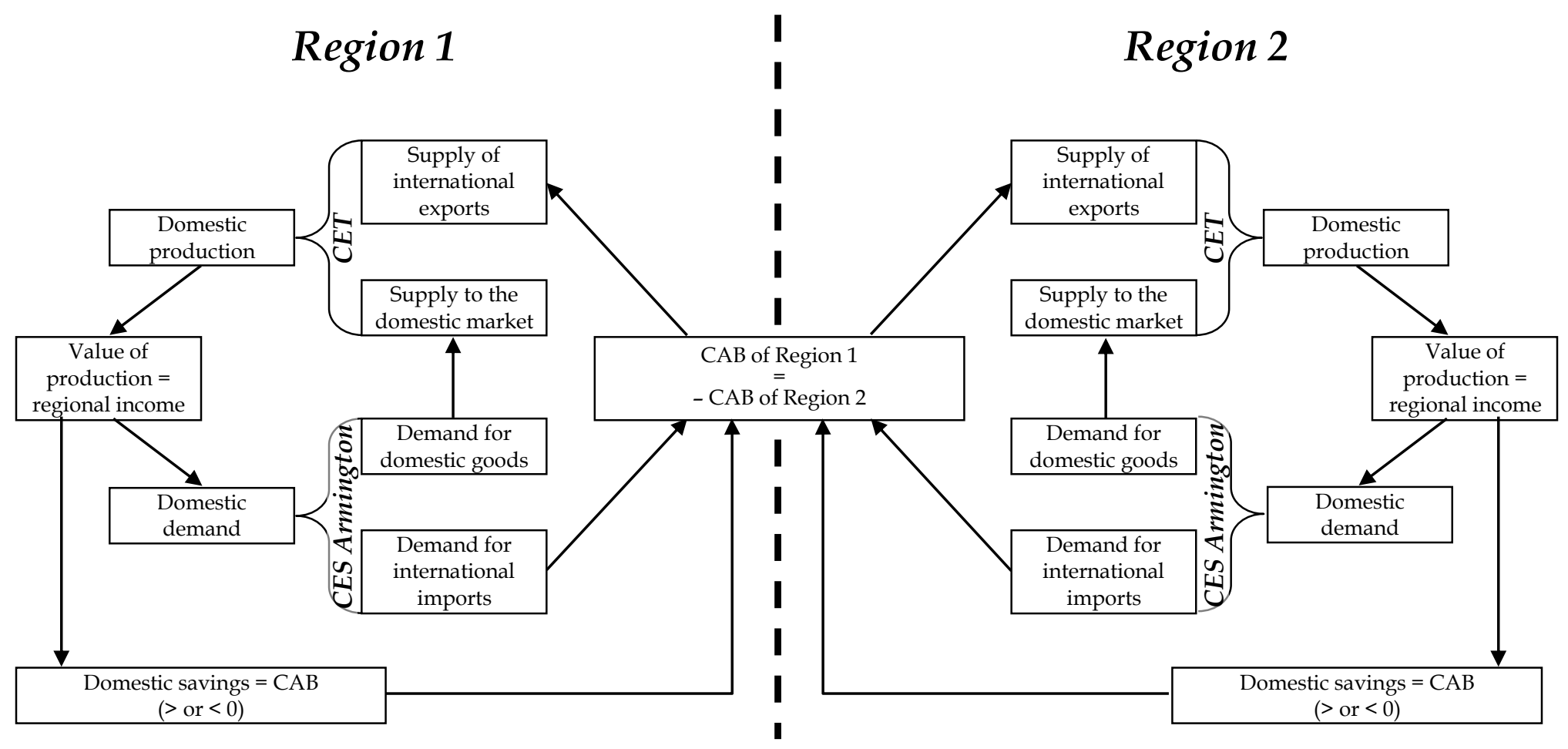

Figure 1. Skeleton model of international trade 
At the top of Figure 1, domestic production is sold on the domestic market or exported, following a CET function. The value of domestic production constitutes the regional agent's income, which is divided between domestic demand, and domestic savings (which may be negative). Domestic demand is distributed following a CES function between domestic production and imports. The imperfect-substitutability Armington hypothesis is what makes it possible to have both imports and exports even if there is a single good. Each region's current account balance (CAB) is given by the value of exports, minus the value of imports. Given that investment is subsumed in consumption, the CAB is equal to domestic savings. Also, in the particular case of two regions, one region's CAB is equal to minus the other's.

Table 1 lists the Model 1 variables, and Table 2 lists the equations (for a more detailed presentation, see Appendix A, supplemental online material). The regions are designated by subscripts $z, z j, z j j \in\{1, \cdots, N\}$ (a different style of subscripting might use $i, j$, and $k$, rather than $z, z j$, and $z j j)$. For instance, in variables $I M_{z j, z}$, $E X_{z, z j}$, and $P W_{z, z j}$, the first subscript designates the region of origin, and the second the region of destination of goods in trade flows. This physical flow fromto convention for subscript order is applied throughout the model. Also note that $I M_{z, Z}=0, E X_{Z, Z}=0$, and $P W_{z, Z}$ is undefined, which reflects the fact that there is no international trade between a country and itself. ${ }^{12}$

Model 1 being a very simple model, it is easy to verify its homogeneity analytically. Indeed, for any set of variable values that solves equations (3)-(18), a new set of variable values obtained by multiplying all prices and nominal values by some positive constant $\lambda$ is also a solution. Homogeneity implies that in the model, prices (and nominal values) are defined only up to a factor of proportionality, so that the modeler must choose a numéraire and set its level.

The number of variables in the model is $11 N+3 N(N-1)$, where $N$ is the number of regions. The number of equations in Table 2 is $12 N+1+3 N(N-1)$ equations. The model appears to be overdetermined. But, as we shall see, it is not.

\footnotetext{
12 In a real global model, the world is divided into regions, some of which include more
} than one country: then, international trade within a multi-country region is possible. 
Table 1. Model 1 variables

\begin{tabular}{|c|c|c|c|}
\hline & & & Number \\
\hline \multicolumn{4}{|c|}{ Volumes } \\
\hline$X S_{Z}$ & Domestic production in region $z$ & \multicolumn{2}{|c|}{$N$} \\
\hline$Q_{Z}$ & Domestic demand for the composite good in region $z$ & \multicolumn{2}{|c|}{$N$} \\
\hline$D_{z}$ & Domestic demand for the locally produced good in region $z$ & \multicolumn{2}{|c|}{$N$} \\
\hline$I M_{z j, Z}$ & Imports from region $z j$ by region $z$ & \multicolumn{2}{|c|}{$N(N-1)$} \\
\hline$I M T_{Z}$ & Total imports of region $z$ & \multicolumn{2}{|c|}{$N$} \\
\hline$E X_{Z, Z j}$ & Exports by region $z$ to region $z j$ & \multicolumn{2}{|c|}{$N(N-1)$} \\
\hline$E X T_{Z}$ & Total exports of region $z$ & \multicolumn{2}{|c|}{$N$} \\
\hline \multicolumn{4}{|l|}{ Prices } \\
\hline$P_{Z}$ & Producer price in region $z$ & \multicolumn{2}{|c|}{$N$} \\
\hline$P L_{Z}$ & Market price of local product in region $z$ & \multicolumn{2}{|c|}{$N$} \\
\hline$P C_{z}$ & Price of the composite good in region $z$ & \multicolumn{2}{|c|}{$N$} \\
\hline$P M T_{Z}$ & Price of composite imports in region $z$ & \multicolumn{2}{|c|}{$N$} \\
\hline$P X T_{Z}$ & Price of composite exports of region $z$ & \multicolumn{2}{|c|}{$N$} \\
\hline$P W_{z, z j}$ & $\begin{array}{l}\text { World price of exports from region } z \text { to } z j \text {, in terms of the } \\
\text { international currency }\end{array}$ & \multicolumn{2}{|c|}{$N(N-1)$} \\
\hline \multicolumn{4}{|c|}{ Nominal value variables } \\
\hline$C A B_{Z}$ & Current account balance of region $z$ & \multicolumn{2}{|c|}{$N$} \\
\hline \multirow{3}{*}{\multicolumn{2}{|c|}{ Total number of variables }} & \multirow{2}{*}{\multicolumn{2}{|c|}{$11 N+3 N(N-1)$}} \\
\hline & & & \\
\hline & & \multicolumn{2}{|r|}{ Number } \\
\hline & $C A B_{Z}=P_{Z} X S_{Z}-P C_{Z} Q_{Z}$ & (3) & $N$ \\
\hline & $C A B_{Z}=P X T_{Z} E X T_{Z}-P M T_{Z} I M T_{Z}$ & $(4)$ & $N$ \\
\hline$X S_{Z}=$ & $\left.\beta_{Z} D_{z}^{\kappa_{z}}+\left(1-\beta_{z}\right) E X T_{z}{ }^{\kappa_{z}}\right]^{\frac{1}{\kappa_{z}}}$ where $\kappa_{z}=\frac{\tau_{z}+1}{\tau_{z}}, 0<\tau_{z}<\infty$ & (5) & $N$ \\
\hline
\end{tabular}


Journal of Global Economic Analysis, Volume 2 (2017), No. 1, pp. 215-324.

Table 2. Model 1 equations (continued)

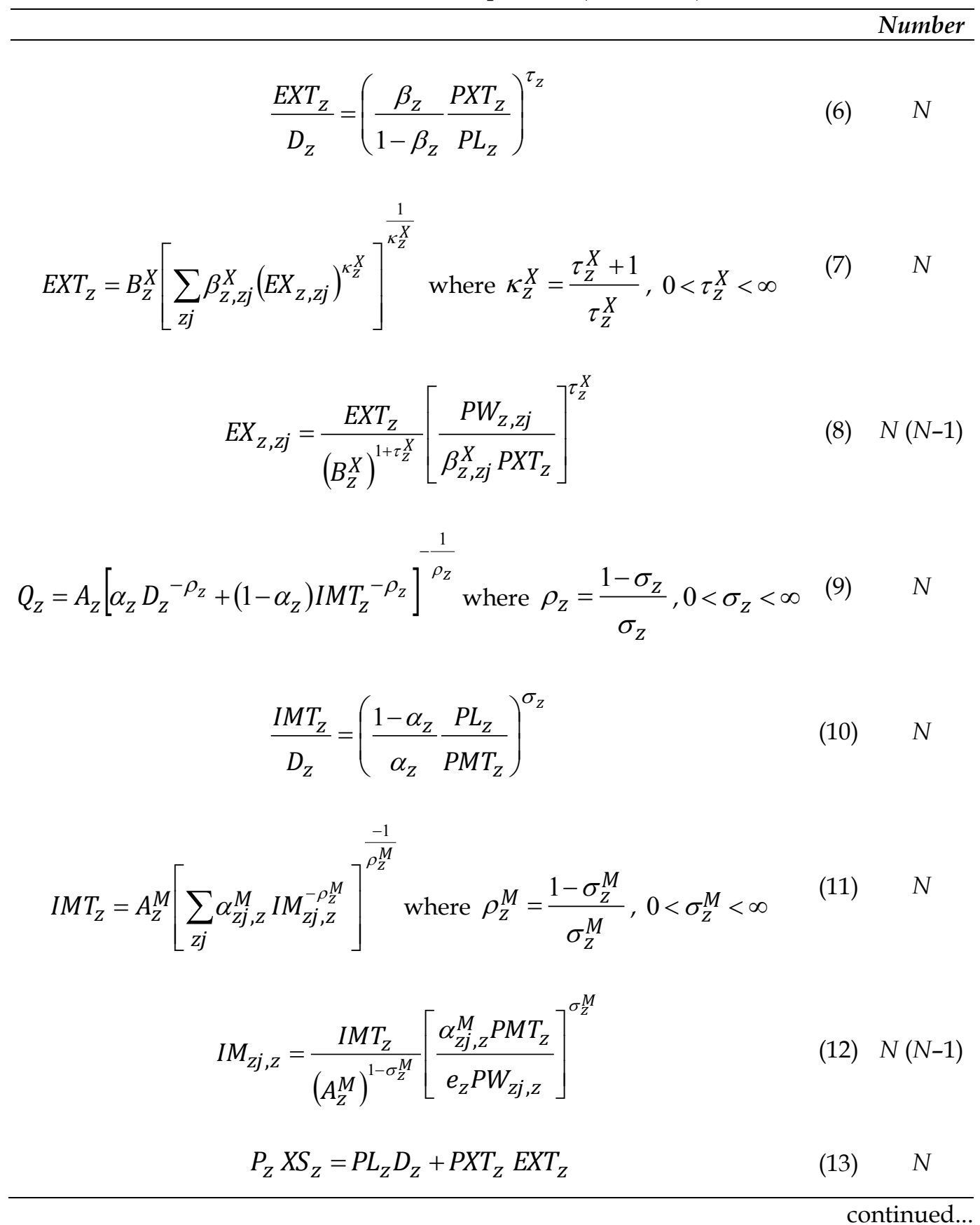

continued... 
Table 2. Model 1 equations (continued)

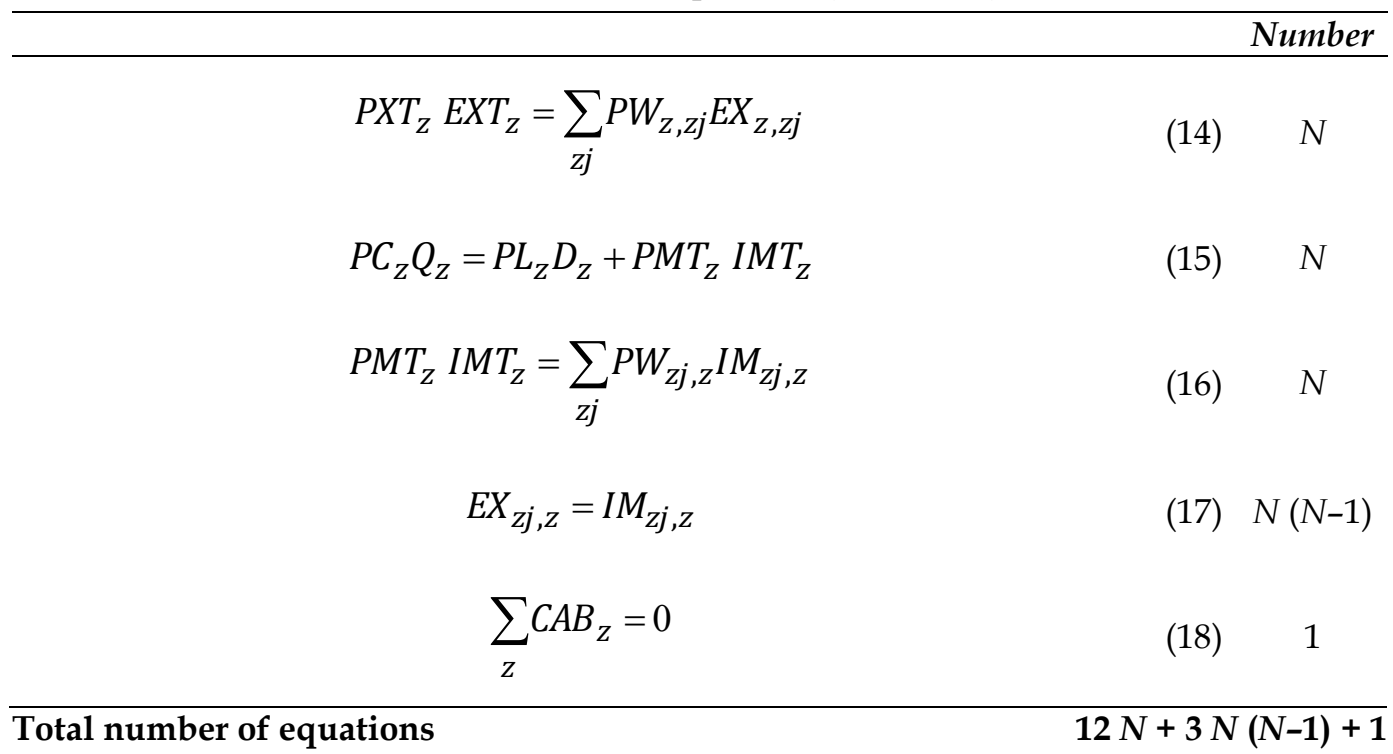

\section{Redundant equations and reduction of the model: Model 2}

How many variables and equations are there in the model? If the number of independent equations is greater than the number of variables, then the model is over-determined and infeasible. On the other hand, if the number of independent equations is less than the number of variables, then some variables have to be made exogenous and fixed in the closure. So it is necessary to identify and eliminate redundant equations from the model.

From Tables 1 and 2, Model 1 appears to have $11 N+3 N(N-1)$ variables: 11 (groups of) variables indexed in $z$, and 3 (groups of) variables indexed in $z, z j$. The equation count is $12 N+3 N(N-1)+1$ : 12 (groups of) equations indexed in $z, 3$ (groups of) equations indexed in $z, z j$, plus the single equation (18). So the model appears to have $N+1$ equations too many. However, as we demonstrate in Appendix B (supplemental online material), several equations are redundant: equations (7) and (8) together imply (14), which is therefore redundant; equations (11) and (12) together imply (16), which is therefore redundant; equations (4) and (17) together imply (18), which is therefore redundant; equations (13), (15) and (3) together imply (4), which is therefore redundant.

In addition, it is shown in Appendix B that Walras' Law can take the following form in our model: if equation (3) is satisfied for $N-1$ regions, then it is also satisfied for the $N^{\text {th }}$ one. Therefore, one equation of the set (3) may be discarded as redundant. So we can arbitrarily pick some region zleon, zleon $\in\{1, \cdots, N\}$ (zleon is a mnemonic for Léon Walras), and remove equation (3) for that single region. Note that with the removal of the equation relating to zleon, the variable $C A B_{\text {zleon }}$ 
no longer appears in the model, but its value may be computed using the suppressed equation.

Some modelers sidestep the task of seeking out and eliminating redundant equations by changing the constrained nonlinear system (CNS) into a nonlinear programming (NLP) problem with a dummy objective variable set equal to a constant. This is risky, because the solver will find a solution even if the model misses a closure equation, which usually implies that the solution produced by the solver depends on the initial values of the variables, because when a closure equation is missing, the solution is generally not unique. This point is illustrated with the help of a simple example in Appendix C (supplemental online material).

We now rewrite the model, discarding redundant equations. After eliminating equations (4), (14), (16) (18), and one equation of the set (3), we are left with Model 2. The equations in Model 2, listed in Table 3, number $9 N-1+3 N(N-1)$, where $N$ is the number of regions. The list of variables is the same as for Model 1, except that $C A B_{\text {zleon }}$ no longer appears in the model; it is determined implicitly. Since it no longer appears in the model, $C A B_{\text {zleon }}$ is not to be counted as a model variable, even if it appears in Table 3. So the reformulated model has $11 N-1+3 N(N-1)$ variables. Thus, Model 2 is underdetermined, it is $2 N$ equations short. So the overdetermined appearance of Model 1 is nothing more than an illusion caused by the presence of redundant equations. Consequently, the closure of Model 2 will require adding $2 \mathrm{~N}$ constraints, including fixing the numéraire. We also note that the homogeneity of Model 2 can be confirmed by examining it analytically in the same way as that of Model 1. 
Table 3. Model 2 equations

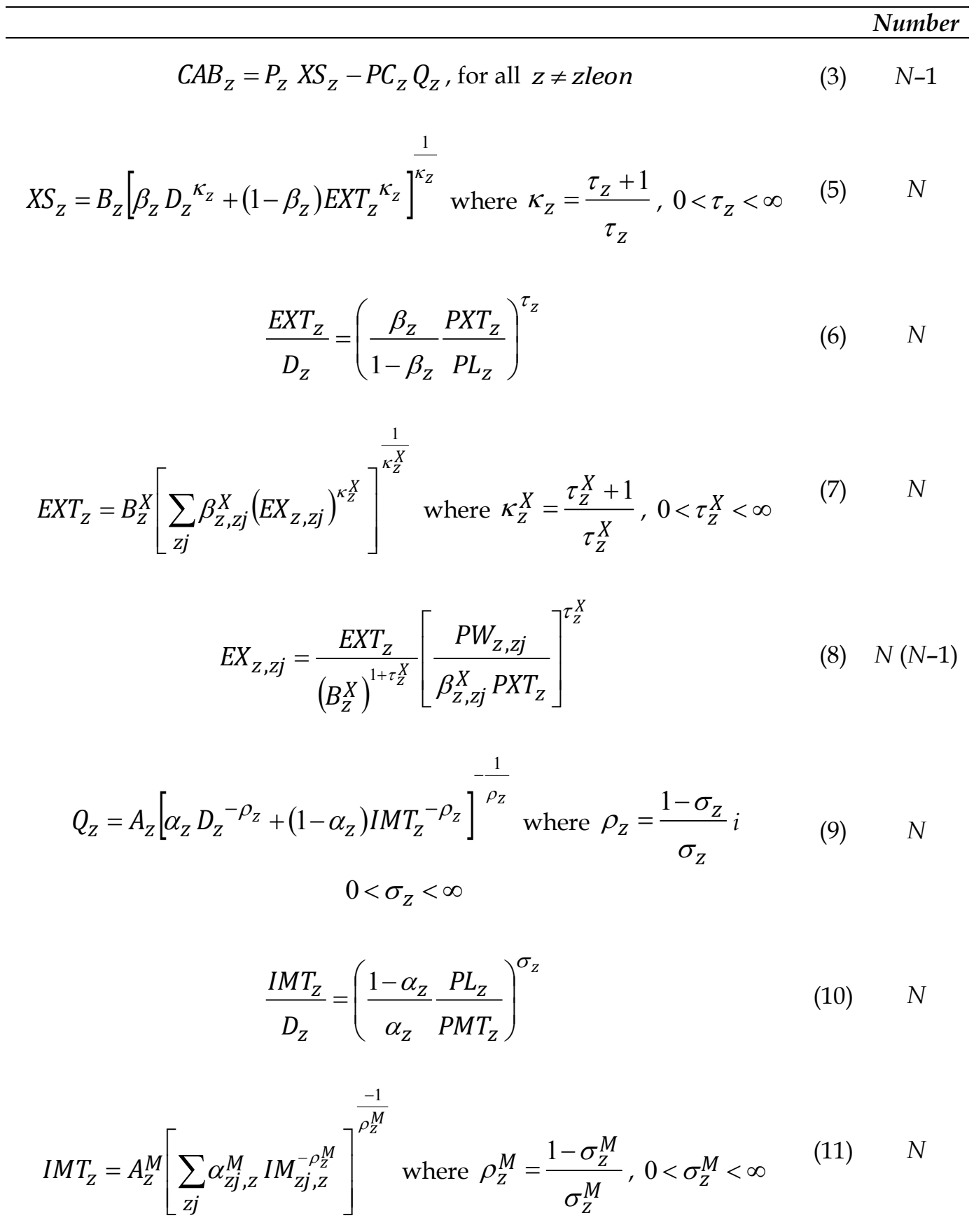


Table 3. Model 2 equations (continued)

\begin{tabular}{|c|c|c|}
\hline & & Number \\
\hline$I M_{z j, z}=\frac{I M T_{z}}{\left(A_{z}^{M}\right)^{1-\sigma_{z}^{M}}}\left[\frac{\alpha_{z j, z}^{M} P M T_{z}}{P W_{z j, z}}\right]$ & (12) & $N(N-1)$ \\
\hline$P_{z} X S_{Z}=P L_{z} D_{z}+P X T_{Z} E X T_{z}$ & (13) & $N$ \\
\hline$P C_{z} Q_{Z}=P L_{z} D_{z}+P M T_{z} I M T_{z}$ & (15) & $N$ \\
\hline$E X_{z j, z}=I M_{z j, z}$ & (17) & $N(N-1)$ \\
\hline
\end{tabular}

Total number of equations

$9 N-1+3 N(N-1)$

\section{Closure of Model 2}

Reviewing the list of variables in Table 1, it might appear that there are several candidates for being fixed in the closure rule. Here we shall go through the list critically, discarding possibilities that are incorrect in the general equilibrium framework. Throughout the review process, we maintain the neoclassical fullemployment hypothesis, which is itself a closure rule, so that we restrict our attention to neoclassical closures. ${ }^{13}$ With production factors in fixed supply, in our simple model, the neoclassical full-employment hypothesis implies that production is fixed: $X S_{Z}=\overline{X S}_{Z}$.

This adds $N$ constraints to the model, leaving $N$ degrees of freedom.

\section{5..1 Neoclassical closure with fixed current account balances}

In this section, we see how fixing $C A B_{Z}$ makes the model non-homogenous, and how homogeneity can be preserved by fixing a pseudo-volume variable $C A B X_{Z}$, the definition of which is not unique, however. Finally, we examine the substantive implications of fixing $C A B_{Z}$ beyond the issue of homogeneity.

We begin with a closure rule that is very common in trade models ${ }^{14}$, which consists in fixing current account balances for $z \neq z l e o n$, in addition to fixing the

${ }^{13}$ It would have been possible to consider non-neoclassical closures, such as fixing $Q_{Z}$. However, our goal is to illustrate the mathematical logic underlying the selection of closure rules, and restricting our attention to neoclassical closures makes the exposition more compact.

${ }^{14}$ Recall however that there are several models with endogenous current account balances, as mentioned in a footnote in the Introduction. 
numéraire. This adds $(N-1)+1=N$ constraints to the model, making it square. However, fixing $C A B_{Z}$ makes the model non-homogenous. The reason is that $C A B_{Z}$ is a nominal variable, and if $C A B_{Z}$ is fixed, the constraint it represents in real terms depends on the choice of numéraire and its value.

Formally, fixing $C A B_{z}, z \neq$ zleon, means fixing $N-1$ elements of the vector of nominal variables $(\mathbf{n})$ in the $\{\mathbf{q}, \mathbf{p}, \mathbf{n}\}$ triplet which represents a model solution (see section 2.1). Now, homogeneity requires that if two simulations starting from the same set of benchmark values $\{\overline{\mathbf{q}}, \overline{\mathbf{p}}, \overline{\mathbf{n}}\}$, with different numéraires, yield solutions $\{\mathbf{q}, \mathbf{p}, \mathbf{n}\}$ and $\left\{\mathbf{q}^{\prime}, \mathbf{p}^{\prime}, \mathbf{n}^{\prime}\right\}$ respectively, then the solutions must satisfy the relationship $\left\{\mathbf{q}^{\prime}, \mathbf{p}^{\prime}, \mathbf{n}^{\prime}\right\}=\{\mathbf{q}, \lambda \mathbf{p}, \lambda \mathbf{n}\}$ for some $\lambda>0$. If $N-1$ elements of the vector of nominal variables (n) are fixed, the relationship can hold only for $\lambda=1$.

Therefore, in a closure that preserves model homogeneity, current account balances must be fixed in real terms. One way to do this is to fix

$$
C A B_{Z}=P W I N D E X C A B X_{Z}
$$

where $C A B X_{Z}$ is fixed in the closure, and

$$
P W I N D E X=\sqrt{\frac{\sum_{z} \sum_{z j} P W_{z, z j} E X_{z, z j}^{O}}{\sum_{z} \sum_{z j} P W_{z, z j}^{O} E X_{z, z j}^{O}} \frac{\sum_{z} \sum_{z j} P W_{z, z j} E X_{z, z j}}{\sum_{z} \sum_{z j} P W_{z, z j}^{O} E X_{z, z j}}}
$$

is a Fisher index of bilateral trade prices, and superscript $O$ designates benchmark values (equivalently, one could substitute $I M_{z, z j}$ for $E X_{z, z j}$ and $I M_{z, z j}^{O}$ for $E X_{z, z j}^{O}$ in (20)).

By inverting (19) to obtain

$$
C A B X_{Z}=C A B_{Z} / P W I N D E X
$$

it is manifest that $C A B X_{Z}$ is a pseudo-volume variable. The introduction of $C A B X_{Z}$ and equation (21) adds $N+1$ variables, because it re-introduces $C A B_{\text {zleon }}$ into the model, and $N$ equations. The introduction of PWINDEX adds one variable and one equation to the model. So the expanded model now has $N+1$ additional equations, for a total of $10 N+3 N(N-1)$ equations, and $N+2$ additional variables, for a total of $12 N+1+3 N(N-1)$ variables, leaving $2 N+1$ degrees of freedom. With $X S_{Z}$ and $C A B X_{Z}$ fixed ( $2 N$ variables), there is a single degree of freedom left. It is used to set the numéraire.

The user may pick any one price from one of the sets $P_{z}, P L_{z}, P C_{Z}, P M T_{Z}, P X T_{Z}$ , or $P W_{z, z j}$, or $P W I N D E X$. Running a simulation with different numéraires will 
yield solutions where the volume variables are equal, and prices and nominal value variables are all proportional (relative prices are the same).

There are of course other ways to define $C A B X_{Z}$. Among the alternatives is the possibility of using another trade-price index, such as a Laspeyres index of bilateral trade prices, or of regions' aggregate import prices, or of regions' aggregate export prices; Paasche or any other type of price indexes could also be used. One could even define the "real" current account balance on the basis of a price that is not directly related to trade, such as, for example, the producer price in some particular region selected as a "reference region". Let the reference region be designated by the index $z r, z r \in\{1, \cdots, N\}$ (the index used for the reference region is $z r$, to indicate that the reference region may be different from region zleon which is excluded from equation (3) under Walras' Law). Then $C A B X_{Z}$ can be defined as

$$
C A B X_{z}=C A B_{z} / P_{z r}
$$

where $P_{z r}$ is the producer price in the reference region.

It must be kept in mind, however, that the denominator that defines $C A B X_{Z}$ must be the same for all regions $z$. It would be an error, for example, to define $C A B X_{Z}=C A B_{Z} / P_{Z}$, where the denominator is specific to each region. In that case, the implicit solution value of $C A B X_{\text {zleon }}$ will be different from its fixed closure value. Since $C A B X_{\text {zleon }}$ does not appear in the model, GAMS will find a solution. But the equation left implicit on account of Walras' Law will be violated, as well as one or more of the redundant equations (4), (14), (16), and (18).

No matter how $C A B X_{Z}$ is defined, provided that definition is admissible, the choice of the numéraire and its value is arbitrary. However, the way $C A B X_{Z}$ is defined does matter! That is because different variants of $C A B X_{Z}$, when they are fixed in the closure, impose different constraints in real terms. Indeed, $C A B X_{Z}$ is a pseudo-volume variable, which does not have a clear-cut, unique definition. Although choosing a particular variant of $C A B X_{Z}$ is not equivalent to choosing any other, none of the possible specifications of $C A B X_{Z}$ stands out as the natural one. 15

It might be tempting to sidestep the issue of defining $C A B X_{Z}$ while maintaining model homogeneity with respect to changes in the value of the numéraire by fixing

$$
C A B_{Z}=v C A B X_{Z}
$$

\footnotetext{
15 This issue does not arise in models such as GTAP where the current account balance is endogenous, although the definition of $C A B X_{Z}$ does matter for interpretation.
} 
where $v$ is the numéraire; the value of $C A B X_{Z}$ fixed in the closure is usually $C A B_{Z}^{O}$ .${ }^{16}$ Indeed, with this definition, the value of $C A B_{Z}$ automatically adjusts to any change in the value of the numéraire. Note that this approach is equivalent to defining $C A B X_{Z}$ as

$$
C A B X_{Z}=C A B_{Z} / v
$$

and fixing it in the closure. This shows that the approach in (23) implicitly defines $C A B X_{Z}$ in terms of the numéraire, so that changing the commodity that serves as the numéraire good modifies the closure in real terms. In other words, the model is homogenous with respect to changes in the value of the numéraire, but not with respect to changes in the choice of the numéraire good.

The following example illustrates this point. The model was run three times in GAMS17; in each run, the model is parametrized, the benchmark solution is computed, and a simulation is performed (in this case, an increase in Region 3's resources/output). All three runs use a fixed nominal $C A B_{z}$ closure. In the first, the numéraire is PWINDEX and it is fixed at 1. This, as we have seen, yields identical results to a fixed $C A B X_{Z}$ closure with the same numéraire. In the second run, the value assigned to the numéraire PWINDEX is set at 2, and the value of fixed nominal $C A B_{z}$ is doubled accordingly. In the third run, Region 3 is designated as the reference region, and the numéraire is changed to $P_{z r}$.

Table 4. SAM and calibrated values (numéraire PWINDEX =1)

\begin{tabular}{lrrrr}
\multicolumn{5}{c}{ Social accounting matrix (benchmark) } \\
\hline & Reg1 & Reg2 & Reg3 & Tot \\
\hline Reg1 & 100 & 10 & & $\mathbf{1 1 0}$ \\
Reg2 & 15 & 50 & 10 & $\mathbf{7 5}$ \\
Reg3 & 5 & & 30 & $\mathbf{3 5}$ \\
CAB & -10 & 15 & -5 & \\
\hline Tot & $\mathbf{1 1 0}$ & $\mathbf{7 5}$ & $\mathbf{3 5}$ & $\mathbf{2 2 0}$ \\
\hline
\end{tabular}

Benchmark Volumes

Benchmark Prices

\footnotetext{
16 This strategy is applied in the Globe model (McDonald and Thierfelder, 2016), and it is used by van der Mensbrugghe (2010, see p.28-29).

${ }_{17}$ The GAMS program which was actually used was not Model 2, but Model 3 with the FE closure and exchange rates set at 1 (see 6.2.1), which yields solutions numerically identical to Model 2's.
} 


\begin{tabular}{lrrr}
\hline & Reg1 & Reg2 & Reg3 \\
\hline DD & 100 & 50 & 30 \\
EXT & 10 & 25 & 5 \\
IMT & 20 & 10 & 10 \\
XS & 110 & 75 & 35 \\
Q & 120 & 60 & 40 \\
\hline \multicolumn{4}{c}{ Bilateral trade volumes - Benchmark } \\
\hline \multicolumn{5}{c}{ Reg1 } \\
\hline Reg1 & Reg2 & Reg3 \\
Reg2 & 15 & 10 \\
Reg3 & 5 & & 10 \\
\hline Source: Author cal & & \\
\end{tabular}

\begin{tabular}{lrrr}
\hline & Reg1 & Reg2 & Reg3 \\
\hline PL & 1 & 1 & 1 \\
P & 1 & 1 & 1 \\
PC & 1 & 1 & 1 \\
PMT & 1 & 1 & 1 \\
PXT & 1 & 1 & 1 \\
\hline
\end{tabular}

Source: Author calculations.

Bilateral trade prices - Benchmark

\begin{tabular}{lrrr}
\hline & Reg1 & Reg2 & Reg3 \\
\hline Reg1 & & 1 & \\
Reg2 & 1 & & 1 \\
Reg3 & 1 & & \\
\hline
\end{tabular}

With PWINDEX $=2$, all benchmark volumes are the same as with PWINDEX $=1$ , and all prices are doubles. When the numéraire is $P_{z r}=1$, with Region 3 as the reference region, all benchmark values are the same as with $P W I N D E X=1$. Now let us compare the simulation-to-benchmark ratios (Table 5). 
Journal of Global Economic Analysis, Volume 2 (2017), No. 1, pp. 215-324.

Table 5. Simulation/benchmark ratios using different numéraires

\begin{tabular}{|c|c|c|c|c|c|c|c|c|c|}
\hline & \multicolumn{3}{|c|}{ PWINDEX = 1} & \multicolumn{3}{|c|}{$P W I N D E X=2$} & \multicolumn{3}{|c|}{$\begin{array}{c}\text { Ref. region }=3, \\
P_{z r}=1\end{array}$} \\
\hline & Reg1 & Reg2 & Reg3 & Reg1 & Reg2 & Reg3 & Reg1 & Reg2 & Reg3 \\
\hline \multicolumn{10}{|c|}{ Volumes } \\
\hline $\mathrm{DD}$ & 0.997 & 1.002 & 1.576 & 0.997 & 1.002 & 1.576 & 0.990 & 1.026 & 1.571 \\
\hline EXT & 1.032 & 0.995 & 1.543 & 1.032 & 0.995 & 1.543 & 1.093 & 0.947 & 1.575 \\
\hline IMT & 1.061 & 1.032 & 1.118 & 1.061 & 1.032 & 1.118 & 1.038 & 1.093 & 1.058 \\
\hline XS & 1.000 & 1.000 & 1.571 & 1.000 & 1.000 & 1.571 & 1.000 & 1.000 & 1.571 \\
\hline Q & 1.007 & 1.007 & 1.454 & 1.007 & 1.007 & 1.454 & 0.998 & 1.037 & 1.433 \\
\hline \multicolumn{10}{|c|}{ Bilateral trade volumes } \\
\hline Reg1 & \multicolumn{3}{|c|}{1.032} & \multicolumn{3}{|c|}{1.032} & \multicolumn{3}{|c|}{1.093} \\
\hline Reg2 & 0.912 & & 1.118 & 0.912 & & 1.118 & 0.872 & & 1.058 \\
\hline Reg3 & 1.543 & & & 1.543 & & & 1.575 & & \\
\hline \multicolumn{10}{|c|}{ Prices } \\
\hline PL & 0.993 & 1.025 & 0.886 & 0.993 & 1.025 & 0.886 & 1.138 & 1.234 & 1.000 \\
\hline $\mathrm{P}$ & 0.995 & 1.024 & 0.885 & 0.995 & 1.024 & 0.885 & 1.143 & 1.218 & 1.000 \\
\hline PC & 0.988 & 1.023 & 0.922 & 0.988 & 1.023 & 0.922 & 1.133 & 1.227 & 1.047 \\
\hline PMT & 0.962 & 1.010 & 1.052 & 0.962 & 1.010 & 1.052 & 1.111 & 1.195 & 1.218 \\
\hline PXT & 1.010 & 1.022 & 0.876 & 1.010 & 1.022 & 0.876 & 1.195 & 1.185 & 1.001 \\
\hline \multicolumn{10}{|c|}{ Bilateral trade prices } \\
\hline Reg1 & \multicolumn{3}{|c|}{1.010} & \multicolumn{3}{|c|}{1.010} & \multicolumn{3}{|c|}{1.195} \\
\hline Reg2 & 1.000 & & 1.052 & 1.000 & & 1.052 & 1.161 & & 1.218 \\
\hline Reg3 & 0.876 & & & 0.876 & & & 1.001 & & \\
\hline
\end{tabular}

Source: Author calculations.

As expected, the nominal $C A B_{Z}$ closure is homogenous with respect to changes in the value given to the numeraire, provided the exogenous value of $C A B_{Z}$ is properly adjusted: model results are equivalent, whether $P W I N D E X=1$ or $P W I N D E X=2$ (left and center panels of Table 5). However, the nominal $C A B_{Z}$ closure is not homogenous with respect to a change in the numéraire good: when the numéraire is changed from PWINDEX to $P_{z r}=1$, the simulation results are different, even if all benchmark values are the same as in the first run (right-hand side panel of Table 5).

\subsection{Neoclassical closure with a set of fixed volume variables}

In this section we extend the formal discussion of closures to systematically examine other possibilities, only to conclude that they are not feasible. Specifically, if $\overline{X S}_{Z}$ is fixed, it is infeasible to fix another set of volume variables. We shall try 
to understand why, and highlight the fact that selecting closure rules cannot be reduced to counting equations and variables.

Model 2 has 6 sets of volume variables in addition to $X S_{z}$ (See Table 1: Model 1 and Model 2 have the same sets of variables). Fixing $X S_{Z}=\overline{X S}_{Z}$ is the form that the neoclassical full-employment hypothesis takes in the skeleton model. We now assert that, in this model, if $\overline{X S}_{z}$ is fixed, it is infeasible to fix another set of volume variables. This can be illustrated in a 2-region version of Model 2; the two-region version of Model 2 is detailed in Appendix H. It consists of equations (3) (for $z \neq$ zleon ), (5), (6), (9), (10), (13), (15) and (21) ${ }^{18}$, together with a trade-price index re-defined for the two-region case

$$
\text { PWINDEX }=\sqrt{\frac{\sum_{Z} P M T_{Z} I M T_{Z}^{O}}{\sum_{Z} P M T_{Z}^{O} I M T_{Z}^{O}} \frac{\sum_{Z} P M T_{Z} I M T_{Z}}{\sum_{Z} P M T_{Z}^{O} I M T_{Z}}}
$$

and the two-region equilibrium conditions

$$
\begin{aligned}
& E X T_{Z j}=I M T_{Z} \\
& P X T_{z j}^{*}=P M T_{Z}^{*}
\end{aligned}
$$

From the 2-region version of Model 2, consider the sub-model consisting of equations (5),(9), and (26). Let us call this sub-model the Q-Model (Q for quantity). With two regions, there are 6 equations and 10 variables in the Q-model: $X S_{Z}, D_{Z}$ , $E X T_{Z}, Q_{Z}, I M T_{Z}$. In principle, it should be possible to fix 4 of them, among which $\overline{X S}_{z}$. Figure 2 summarizes the two-region Q-model. The position of the CET production frontiers in the north-west and south-east quadrants is determined by the fixed $\overline{X S}_{z}$. As long as the $Q_{z}$ are not fixed, the CES indifference curves in the north-east and south-west quadrants are free to move inward or outward. Setting a pair of $D_{z}, E X T_{Z}$, or $I M T_{z}$ specifies a point on each of the CET frontiers defined by $\overline{X S}_{Z}$. Drawing perpendicular lines from these two points traces a rectangle. The

\footnotetext{
${ }^{18}$ Equation (3) is the CAB definition; (5) is the CET transformation between domestic sales and exports, and (6) is the relative supply function; (9) is the CES aggregator between the domestic good and imports, and (10) is the relative demand function; (13) equates the value of production to the sum of the value of sales on the domestic market and exports; (15) equates total expenditures to the sum of purchases on the domestic market and the value of imports; (19) is the definition of $C A B X_{Z}$.
} 
solution corresponds to the values of $Q_{z}$ that make the indifference curve pass through the north-east and south-west corners of the rectangle.

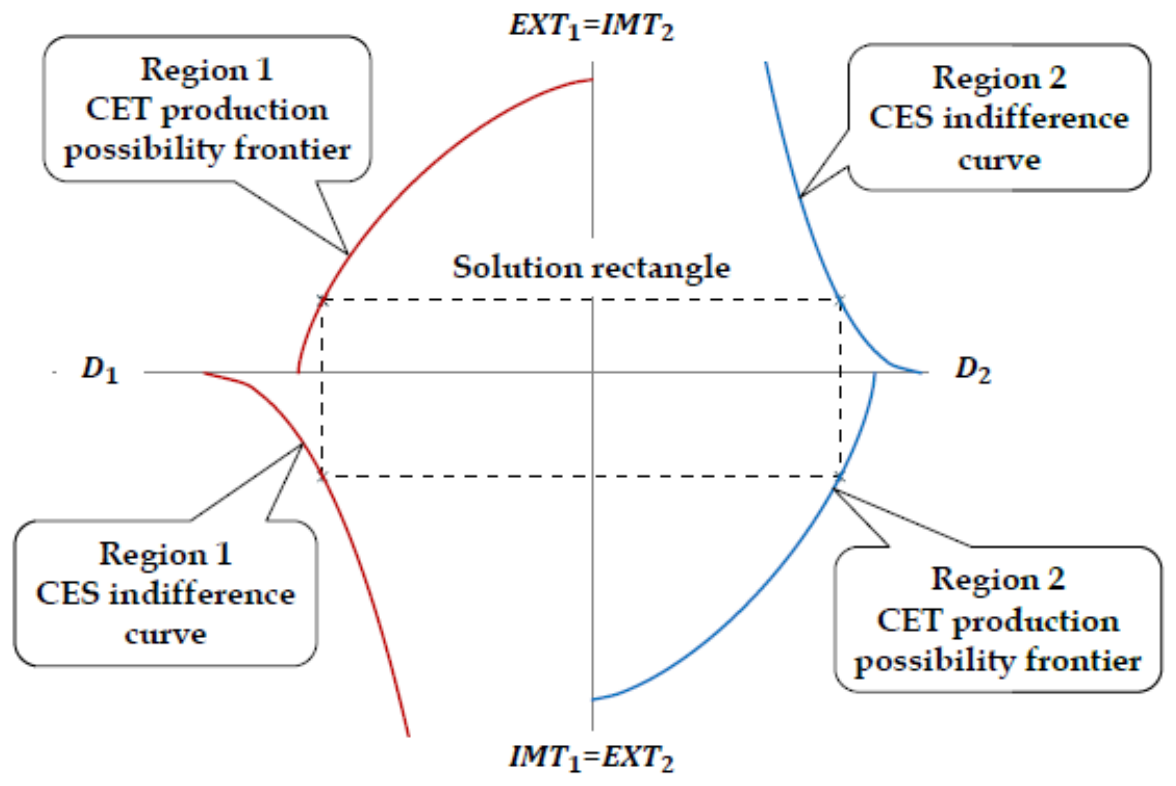

Figure 2. The two-region Q-Model

It is quite obvious that if, a contrario, the $Q_{z}$ are fixed rather than the $D_{z}, E X T_{z}$ , or $I M T_{Z}$, there is no guarantee that a solution rectangle exists. Therefore, given $\overline{X S}_{Z}, Q_{Z}$ cannot be fixed arbitrarily. ${ }^{19}$ And if a solution exists, it may well not be unique. Let us examine this more closely with the two-region Q-Model. Suppose the $Q_{Z}$ are fixed: $Q_{Z}=\bar{Q}_{Z}$. Then combining equations (5), (9) and (26) yields

${ }^{19}$ This kind of limitation is common in CGE models. Indeed, it was the central issue in Sen (1963). 


$$
\begin{aligned}
I M T_{Z}=\left\{\frac{1}{\left(1-\alpha_{z}\right)}\left(\frac{\bar{Q}_{Z}}{A_{z}}\right)^{-\rho_{z}}\right. \\
\left.-\frac{\alpha_{z}}{\left(1-\alpha_{z}\right)}\left[\frac{1}{\beta_{z}}\left(\frac{\overline{X S}_{z}}{B_{Z}}\right)^{\kappa_{z}}-\frac{\left(1-\beta_{z}\right)}{\beta_{z}} I M T_{z j} \kappa_{z}\right]^{-\frac{\rho_{Z}}{\kappa_{z}}}\right\}^{-\frac{1}{\rho_{z}}}
\end{aligned}
$$

(see Appendix I, supplemental online material). Given $\overline{X S}_{z}$ and $\bar{Q}_{Z}$, the pair of equations (28) can be solved for $I M T_{Z}$; then $E X T_{Z}$ and $D_{Z}$ follow from (26) and either (9) or (5). The pair of equations (28) $\left(I M T_{2}\right.$ as a function of $I M T_{1}$, and $I M T_{1}$ as a function of $I M T_{2}$ ) are plotted for different values of $\bar{Q}_{Z}$ in the four parts of Figure 3.

The two functions are convex: as a function of the other region's imports, the imports of each region increase at a decreasing rate. The solution, of course, is given by the intersection of the two curves. In the benchmark equilibrium, the curves are tangent at the solution (Figure 3(a)). If the exogenous volume of domestic demand $\bar{Q}_{z}$ is increased in both regions (Figure 3(b)), the curves no longer intersect: there is no solution. If, on the contrary, $\bar{Q}_{z}$ is reduced in both regions (Figure 3(c)), then the solution exists, but it is not unique. Finally, Figure 3(d) illustrates a situation where $\bar{Q}_{z}$ is increased in one region, but reduced in the other; in that particular case, there is a solution, but it is not unique.

This explains why, if one tries to close the model by fixing $Q_{Z}$, then the CONOPT solver cannot find a solution and issues the diagnostic "Pivot too small", which "means that the set of constraints is linearly dependent in the current point and there is no unique search direction for Newton's method so CONOPT terminates". ${ }^{20}$

\footnotetext{
${ }^{20}$ The reader can find this quote through the GAMS Help menu, under "Solver manual". Click on CONOPT and navigate to Appendix A/Miscellaneous Topics/Constrained Nonlinear Systems or Square Systems Of Equations. The author of the CONOPT solver manual is Arne Drud, ARKI Consulting and Development A/S, Bagsvaerd, Denmark (http://www.conopt.com).
} 
Panel 1

Benchmark equilibrium

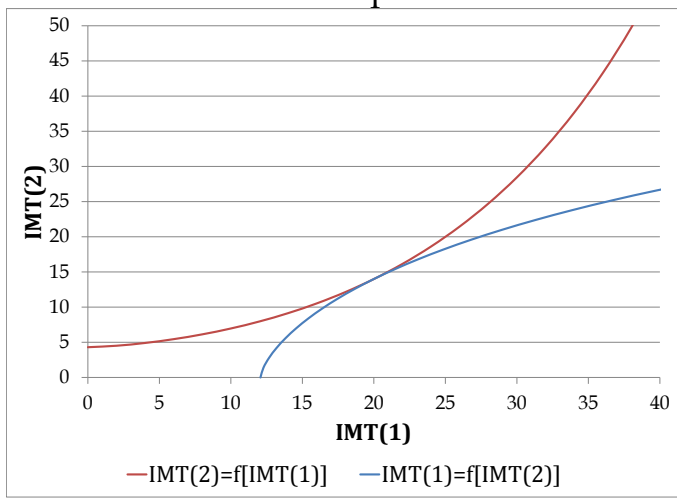

Panel 3

Domestic demand reduction in both regions

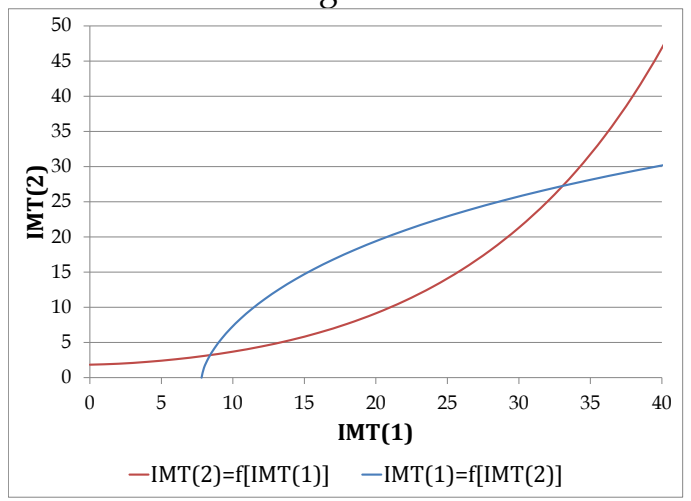

Panel 2

Domestic demand increase in both regions

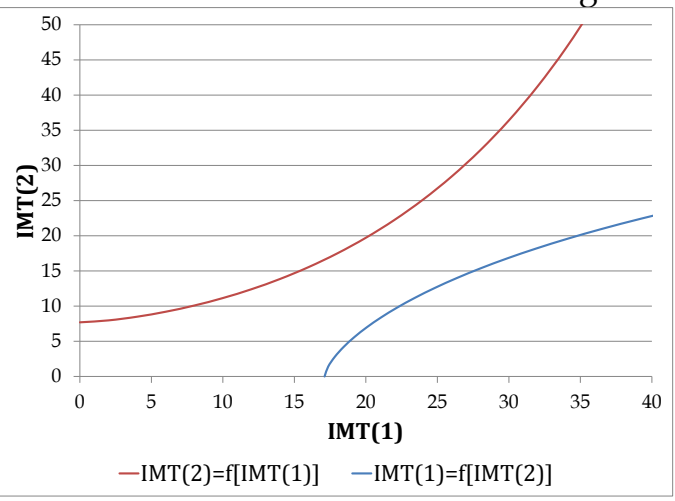

Panel 4

Domestic demand reduction in region 1 , increase in region 2

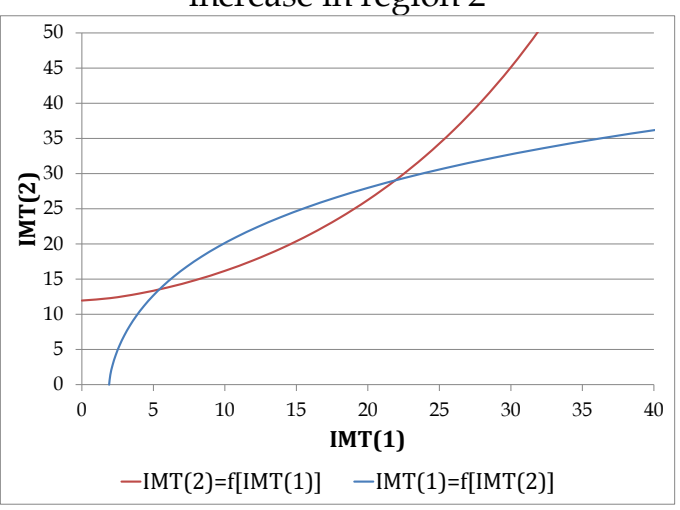

Figure 3. Existence of a solution in the two-region Q-Model

What about closures that would fix $D_{Z}, E X T_{Z}$, or $I M T_{Z}$, in addition to $\overline{X S}_{Z}$ ? We have shown above that such a closure determines all volume variables in the model, using equations (5), (9), and (26). The issue is then whether there exists a set of prices that can make those quantities equilibrium quantities. Given the calibrated values of the model parameters, there is no guarantee that there is such a set of prices. Moreover, if such a set of prices exists, the price equation subsystem becomes degenerate.

To illustrate this, consider once again the two-region model described in Appendix $\mathrm{H}$. After removing the Q-model equations, the rest of the model consists of 14 equations; given the Q-model solution, there are 15 remaining variables, one of which is to be fixed as the numéraire. Separate the 14 equations into two submodels. The core price model (the P-Model) consists of 3 pairs of equations: equations (6), (10), and (27), and it comprises 6 variables: $P L_{Z}, P M T_{Z}$, and $P X T_{Z}$. 
The remaining equations, (3) (for $z \neq$ zleon), (21), (13), (15) and (25), constitute the downstream part of the model, which is readily solved using the solution values of the volume variables in the Q-Model and of the prices in the P-Model. Note, however, that the P-Model is homogenous in prices, as it should be. Therefore, its solution can be defined only up to a factor of proportionality. This implies logically that the 6 equations cannot be independent (the P-Model cannot be of full rank). Indeed, following the development in Appendix J (supplemental online material), the P-Model equations may be combined to yield

$$
\frac{P M T_{Z}}{P M T_{z j}}=\frac{1-\alpha_{z}}{\alpha_{z}}\left(\frac{D_{z}}{I M T_{Z}}\right)^{1 / \sigma_{z}} \frac{\beta_{z}}{1-\beta_{z}}\left(\frac{D_{z}}{E X T_{Z}}\right)^{1 / \tau_{z}}
$$

Write equation (29) explicitely for $P M T_{1} / P M T_{2}$ and for $P M T_{2} / P M T_{1}$, and invert the second, so that the right-hand side of both equations is equal to $P M T_{1} / P M T_{2}$. It follows immediately that the P-Model has a solution only if

$$
\begin{aligned}
& \frac{1-\alpha_{1}}{\alpha_{1}}\left(\frac{D_{1}}{I M T_{1}}\right)^{1 / \sigma_{1}} \frac{\beta_{1}}{1-\beta_{1}}\left(\frac{D_{1}}{E X T_{1}}\right)^{1 / \tau_{1}} \\
& \quad=\frac{\alpha_{2}}{1-\alpha_{2}}\left(\frac{I M T_{2}}{D_{2}}\right)^{1 / \sigma_{2}} \frac{1-\beta_{2}}{\beta_{2}}\left(\frac{E X T_{2}}{D_{2}}\right)^{1 / \tau_{2}}
\end{aligned}
$$

And if a solution exists, then equation (29) consists of two identical equations and the system is singular, leading to solver diagnostic "Pivot too small". Note that equation (29) is implied by the model, and it therefore remains in force under any closure. What leads to the predicament described here is the fact that all right-hand side variables in equation (29) are hypothesized to have been predetermined in the Q-Model, so that they are treated as constants.

To summarize, we have shown for the two-region version of Model 2 that, given $X S_{Z}=\overline{X S}_{Z}$, it is not possible to close the model using $Q_{Z}=\bar{Q}_{Z}$, nor is it possible to close the model by fixing $D_{z}, E X T_{z}$, or $I M T_{Z}$. I have not been able to formally generalize the demonstration to the $N$-region version of Model 2. But experiments show that the GAMS solver's behavior in the three-region version is the same as in the two-region version. And our close examination of the tworegion Model 2 yields a rather powerful intuition of why the model doesn't solve when one attempts to fix volume variables in addition to $X S_{Z}=\overline{X S}_{Z}$ in the closure (this includes volume variables $E X_{z, z j}$ and $I M_{z, z j}$, which are absent in the tworegion version). The general lesson to be drawn from this exploration is that selecting closure rules cannot be reduced to counting equations and variables. 
Indeed, as was mentioned in an earlier footnote, the choice of variables that can be fixed in the model closure is subject to restrictions in all CGE models no matter how large; that was the central issue in Sen (1963).

Finally, for the sake of completeness, it should be obvious that closing the model by fixing more than one price (the numéraire) in Model 2 is not admissible, because it prevents relative prices from adjusting.

\section{Model with exchange rates: Model 3}

We now expand the model to introduce nominal exchange rates. Initially, these are merely conversion factors to convert values expressed in terms of the international currency into values expressed in terms of domestic currencies. We shall see however that, under certain closure rules, they may be interpreted as price ratios that have economic meaning.

\subsection{Variable transformation}

Let $e_{z}$ be the price of the international currency in terms of region $z^{\prime}$ s domestic currency. All regional prices and nominal values in the model can be expressed in terms of domestic currencies by multiplying the values in terms of the international currency by $e_{z}$. So let $P_{Z}^{*}=e_{Z} P_{Z}$, and define similarly $P L_{z}^{*}, P C_{Z}^{*}$, $P M T_{Z}^{*}, P X T_{Z}^{*}$, and $C A B_{z}^{*}$; the transformation is not applied to $P W_{z, z j}$, which remains expressed in terms of the international currency. Model 3 is the same as Model 2, except that $P_{z}^{*}, P L_{z}^{*}, P C_{z}^{*}, P M T_{z}^{*}, P X T_{z}^{*}$ and $C A B_{z}^{*}$ are substituted for the corresponding variables in terms of the international currency.

By construction, Model 3 is therefore exactly equivalent to Model 2. Indeed, any solution of Model 3 can be converted to a solution of Model 2 by reversing the conversions using $P_{Z}=P_{Z}^{*} / e_{Z}, P L_{Z}=P L_{Z}^{*} / e_{Z}$, etc. This holds for any set of arbitrary $e_{z}>0$. We conclude that the exchange rates in Model 3 are totally arbitrary, or else that they are superfluous. As a matter of fact, several world trade models, such as GTAP (Hertel, 1997) or MIRAGE (Bchir et al., 2002a, 2002b; Decreux and Valin, 2007), are expressed in terms of an international currency and have no exchange rates.

\subsection{Closure}

We now consider the issue of closures in a model with nominal exchange rates. We use an expanded version of Model 3, as we did with Model 2, adding $C A B X_{Z}^{*}$ and PWINDEX and corresponding equations (21) and (20), mutatis mutandis. With exchange rates $e_{z}$ in Model 3 , there are $N$ more variables than in the expanded Model 2, but the same number of equations (which is consistent with the arbitrariness of the $\left.e_{z}\right)$. The expanded Model 3 has 
$10 N+3 N(N-1)$ equations like Model 2, and $13 N+1+3 N(N-1)$ variables (recall that introducing equation (21) re-introduces $C A B_{\text {zleon }}^{*}$ ). There are $3 N+1$ degrees of freedom left, $N$ more than in Model 2. We know that any solution of Model 2 can be extended to Model 3 by converting prices and nominal values from the international currency to regional currencies, that is by multiplying them by exchange rate $e_{z}$. Quite obviously, for this transformation to be valid, there is no restriction on the $e_{z}$ other than being strictly positive. Consequently, any set of conditions which constitutes a proper closure of Model 2, together with an arbitrary set of exchange rates, is also a proper closure of Model 3 (fixing an exchange rate at a value different from its benchmark value merely results in a proportionally equal change in all regional prices and nominal variables; e.g. doubling region $z^{\prime}$ s fixed exchange rate will result in regional prices and nominal variables double their benchmark value).

\subsubsection{Closures with fixed or endogenous exchange rates}

One closure that is frequently used by modelers, which we call the FE closure (for Fixed Exchange rates), is to set the $e_{Z}$ and the $C A B X_{Z}^{*}(2 \mathrm{~N}$ variables fixed in the closure), and to pick some price such as PWINDEX or a reference region's producer price $P_{z r}^{*}$ as the numéraire. As a matter of fact, fixing all exchange rates to 1 makes Model 3 solutions numerically identical to Model 2 solutions.

Another possibility, which we call the FP closure (for Fixed regional Price indexes), is to fix the $C A B X_{Z}^{*}$ and one price, such as $P_{Z}^{*}$, in each region ( $2 N$ variables fixed in the closure), and to take some international price, or the exchange rate of some region picked as the reference region, as the numéraire. Why is it admissible to exogenously set prices other than the numéraire in Model 3, while it was not in Model 2? Because all that matters are the ratios of regional prices to nominal exchange rates (see the variable transformation described in 6.1). In other words, if nominal exchange rates $e_{z}$ are free to move, then fixing some set of regional prices such as $P_{z}^{*}$ does not prevent relative prices from adjusting any more than fixing $e_{z}$ under the FE closure. And that remains true even if $e_{z r}$ is chosen as the numéraire.

However, under the FP closure, since one set of prices (say $P_{z}^{*}$ ) are fixed as regional numéraires, then you cannot fix another regional price (say $P L_{z r}^{*}$ ) in reference region zr as the global numéraire, because then relative prices in region $z r$ are prevented from adjusting. So with the FP closure, if the numéraire is not an exchange rate, then it must be an international price like PWINDEX or even, although it would be somewhat odd, some $P W_{z r, z r j}$ (in which case the numéraire commodity is a particular bilateral trade flow between a pair of reference regions, 
$z r$ and $z r j, z r, z r j \in\{1, \cdots, N\})$. Also note that under the FP closure, regional numéraires may be fixed at arbitrary values, just like nominal exchange rates may be fixed at arbitrary values in the FE closure.

Solutions obtained under the FP closure are readily converted to FE closure solutions and vice-versa. If all regional prices $\left(P_{Z}^{*}, P L_{Z}^{*}, P C_{Z}^{*}, P M T_{Z}^{*}\right.$, and $\left.P X T_{Z}^{*}\right)$ and the nominal value variable $C A B_{z}^{*}$ in the FP solution are divided by the corresponding nominal exchange rate, the values obtained are exactly equal to the regional prices obtained with the FE closure when $e_{z}=1$, as well as to the solution of Model 2 (without exchange rates). ${ }^{21}$

Table 6 compares simulation/benchmark ratios with the FE and FP closures. The simulation consists in an increase in Region 3's resources/output. The table shows that the models are equivalent in that the volume ratios are equal. The bilateral trade price ratios are also equal (trade prices are expressed in the international currency).

${ }^{21}$ The FE and FP equivalence is not driven by the simplifying assumptions of the skeleton model. It is also true of the PEP-w-1, PEP-w-t and PEP-w-t-fin models, with which I have run experiments. 
Table 6. Simulation/benchmark ratios with FE and FP closures

\begin{tabular}{|c|c|c|c|c|c|c|}
\hline & \multicolumn{3}{|c|}{ FE closure } & \multicolumn{3}{|c|}{ FP closure } \\
\hline & Reg1 & Reg2 & Reg3 & Reg1 & Reg2 & Reg3 \\
\hline \multicolumn{7}{|c|}{ Volumes } \\
\hline DD & 0.997 & 1.002 & 1.576 & 0.997 & 1.002 & 1.576 \\
\hline EXT & 1.032 & 0.995 & 1.543 & 1.032 & 0.995 & 1.543 \\
\hline IMT & 1.061 & 1.032 & 1.118 & 1.061 & 1.032 & 1.118 \\
\hline XS & 1.000 & 1.000 & 1.571 & 1.000 & 1.000 & 1.571 \\
\hline Q & 1.007 & 1.007 & 1.454 & 1.007 & 1.007 & 1.454 \\
\hline \multicolumn{7}{|c|}{ Bilateral trade volumes } \\
\hline Reg1 & \multicolumn{3}{|c|}{1.032} & \multicolumn{3}{|c|}{1.032} \\
\hline Reg2 & 0.912 & & 1.118 & 0.912 & & 1.118 \\
\hline Reg3 & 1.543 & & & 1.543 & & \\
\hline \multicolumn{7}{|c|}{ Prices } \\
\hline PL & 1.000 & 1.000 & 1.000 & 1.005 & 0.976 & 1.130 \\
\hline $\mathrm{P}$ & 0.993 & 1.025 & 0.886 & 0.998 & 1.001 & 1.002 \\
\hline PC & 0.995 & 1.024 & 0.885 & 1.000 & 1.000 & 1.000 \\
\hline PMT & 0.988 & 1.023 & 0.922 & 0.993 & 0.999 & 1.043 \\
\hline PXT & 0.962 & 1.010 & 1.052 & 0.967 & 0.987 & 1.189 \\
\hline \multicolumn{7}{|c|}{ Bilateral trade prices } \\
\hline Reg1 & \multicolumn{3}{|c|}{1.010} & \multicolumn{3}{|c|}{1.010} \\
\hline Reg2 & 1.000 & & 1.052 & 1.000 & & 1.052 \\
\hline Reg3 & 0.876 & & & 0.876 & & \\
\hline
\end{tabular}

As for regional prices, we can show that they fulfill condition (1) put forth in section 2.3:

$$
\frac{P_{i, 1}^{C 1, S 1} / P_{i, 0}^{C 1, S 1}}{P_{h, 1}^{C 1, S 1} / P_{h, 0}^{C 1, S 1}}=\frac{P_{i, 1}^{C 2, S 2} / P_{i, 0}^{C 2, S 2}}{P_{h, 1}^{C 2, S 2} / P_{h, 0}^{C 2, S 2}}
$$

Let price variable $h$ be the nominal exchange rate. Identify $[C 1, S 1]$ as the FE closure, and $[C 2, S 2]$ as the FP closure. Then $P_{h, 1}^{C 2, S 2} / P_{h, 0}^{C 2, S 2}$ is equal to 1.005, 0.976, and 1.130 for regions 1,2 and 3 respectively. If we divide all simulation/benchmark price ratios under the FP closure by these exchange rate ratios, we find that indeed, condition (1) is realized (Table 7). 
Table 7. Simulation/benchmark ratios of regional relative prices under FP closure

\begin{tabular}{llll}
\hline & Reg1 & Reg2 & Reg3 \\
\hline $\mathrm{e}$ & 1.000 & 1.000 & 1.000 \\
$\mathrm{PL}$ & 0.993 & 1.025 & 0.886 \\
$\mathrm{P}$ & 0.995 & 1.024 & 0.885 \\
$\mathrm{PC}$ & 0.988 & 1.023 & 0.922 \\
$\mathrm{PMT}$ & 0.962 & 1.010 & 1.052 \\
$\mathrm{PXT}$ & 1.010 & 1.022 & 0.876 \\
\hline \multicolumn{2}{l}{ Source: Author calculations. }
\end{tabular}

The FP closure is the default closure of the Globe model (McDonald and Thierfelder, 2016), and current versions of the PEP standard world CGE models offer the user a choice between the FE and the FP closure (Lemelin, Robichaud, Decaluwé \& Maisonnave, 2013; Lemelin et al., 2014; Lemelin and Robichaud, 2014). But why consider the FP closure? Because it has an interesting interpretation. The fixed regional prices $\left(P_{z}^{*}\right.$ or other) play the role of regional numéraires, and the nominal exchange rate $e_{z}$ is the price of the global numéraire good in terms of the regional one. So in complex models, the FP closure facilitates the analysis of price changes: with regional numéraires, it is easier to distinguish between movements of regional prices relative to each other, and joint movements of regional prices relative to international prices; the latter are reflected in the nominal exchange rates (see Table 6). As a matter of fact, the FE and FP closures mimic a fixed and flexible exchange rate regime respectively. The verb "mimic" points to the fact that, in the idealized frictionless world of most CGE models, the difference between the two is purely formal as we have seen, while in reality, it is substantive. But in simulations, the FE closure solution can be viewed as illustrative of adjustment by inflation or deflation ("internal" devaluation or revaluation; think of Greece in the Euro zone); the FP closure, on the other hand, can be viewed as an illustration of adjustment through devaluation or revaluation of the currency.

\subsubsection{FP closure endogenous exchange rates and real exchange rates}

What is the relationship between the FP closure nominal exchange rates and real exchange rates? In this section, we show that the FP closure exchange rates are indeed nominal, and not real, exchange rates. However, absent changes in other exchange rates, proportional changes in a region's exchange rate are equal to proportional changes in its multilateral real exchange rate.

Chinn $(2006,2008)$ defines the bilateral real exchange rate as $q=s-p+p^{*}$, where $q$ is the logarithm of the real exchange rate, $s$ is the logarithm of the nominal rate (price of the foreign currency in terms of the domestic one), and $p$ and $p^{*}$ are 
logarithms of the domestic and foreign price levels respectively. Let us transpose this definition to our notation. In our model, a bilateral nominal exchange rate is

$$
E_{z, z j}=e_{z} / e_{z j}
$$

Now, $e_{z}$ is the price of the international currency in terms of $z$ 's domestic currency, while $1 / e_{z j}$ is the price of $z j$ 's domestic currency in terms of the international currency, so that $E_{z, z j}$ is the price of region $z j$ 's currency in terms of region $z^{\prime}$ s currency. And, taking antilogs, Chinn's definition can be written as

$$
E_{z, z j}^{R}=E_{z, z j} \frac{P_{z j}^{*}}{P_{z}^{*}}
$$

where $E_{z, z j}^{R}$ is the bilateral real exchange rate, and $P_{z}^{*}$ and $P_{z j}^{*}$ are taken as "regional price levels" (other regional prices could play the role). Given (31), definition (32) is equivalent to

$$
E_{z, z j}^{R}=\frac{P_{z j}^{*} / e_{z j}}{P_{z}^{*} / e_{z}}
$$

And given the variable transformations defined in 6.1,

$$
E_{z, z j}^{R}=\frac{P_{z j}}{P_{z}}
$$

The bilateral real exchange rate is equal to the ratio of the foreign to the domestic price level expressed in terms of the international currency. In our highly simplified model, with a single good and fixed production factors, regional producer prices $P_{z}$ and $P_{z j}$ can be also interpreted as factor prices, so that the bilateral real exchange rate in (34) is the ratio of foreign to domestic factor prices. Note that equation (34) implies that the value of a bilateral real exchange rate is the same in Model 3 as in Model 2, whether the FE or the FP closure is applied.

Under the FP closure, if $P_{z}^{*}$ and $P_{z j}^{*}$ are regional numéraires set equal to 1 , then from (33),

$$
E_{z, z j}^{R}=\frac{e_{z}}{e_{z j}}
$$

Under the FP closure, a bilateral real exchange rate is equal to the ratio of the domestic exchange rate over the exchange rate of the trading partner. Note that, 
in view of (34), the bilateral real exchange rate is independent of the choice of regional numéraire.

In Table 8, bilateral exchange rates are computed from the simulations of Table 6.

Table 8. Bilateral real exchange rates with FE and FP closures

\begin{tabular}{|c|c|c|c|c|c|c|}
\hline & \multicolumn{3}{|c|}{ FE closure } & \multicolumn{3}{|c|}{ FP closure } \\
\hline & Reg1 & Reg2 & Reg3 & Reg1 & Reg2 & Reg3 \\
\hline \multicolumn{7}{|c|}{ Prices - Simulation results } \\
\hline e & 1.000 & 1.000 & 1.000 & 1.005 & 0.976 & 1.130 \\
\hline PL & 0.993 & 1.025 & 0.886 & 0.998 & 1.001 & 1.002 \\
\hline $\mathrm{P}$ & 0.995 & 1.024 & 0.885 & 1.000 & 1.000 & 1.000 \\
\hline PC & 0.988 & 1.023 & 0.922 & 0.993 & 0.999 & 1.043 \\
\hline PMT & 0.962 & 1.010 & 1.052 & 0.967 & 0.987 & 1.189 \\
\hline PXT & 1.010 & 1.022 & 0.876 & 1.016 & 0.998 & 0.991 \\
\hline \multicolumn{7}{|c|}{ Bilateral exchange rates } \\
\hline Reg1 & \multicolumn{3}{|c|}{1.030} & \multicolumn{3}{|c|}{1.030} \\
\hline Reg2 & 0.971 & & 0.864 & 0.971 & & 0.864 \\
\hline Reg3 & 1.124 & & & 1.124 & & \\
\hline
\end{tabular}

Source: Author calculations.

From (35), it is obvious that $e_{Z}$ is not a bilateral real exchange rate. But what about a multilateral real exchange rate? Well, to define a multilateral real exchange rate would require to define a price-level variable for the world. For example, suppose we use a weighted average of regional price levels:

$$
P_{R o W}=\sum_{z j \neq z} w_{z j}\left(P_{z j}^{*} / e_{z j}\right)
$$

Then

$$
\begin{gathered}
E_{z, R o W}^{R}=\frac{P_{R o W}}{P_{z}^{*} / e_{z}} \\
E_{z, R o W}^{R}=\frac{\sum_{z j \neq z} w_{z j}\left(P_{z j}^{*} / e_{z j}\right)}{P_{z}^{*} / e_{z}} \\
E_{z, R o W}^{R}=\sum_{z j \neq z} w_{z j}\left(\frac{P_{z j}^{*} / e_{z j}}{P_{z}^{*} / e_{z}}\right)
\end{gathered}
$$




$$
E_{z, R o W}^{R}=\sum_{z j \neq Z} w_{z j} E_{z, z j}^{R}
$$

The multilateral real exchange rate is a weighted average of the bilateral ones. Then under the FP closure, we have

$$
\begin{aligned}
& E_{z, R o W}^{R}=\sum_{z j \neq z} w_{z j} \frac{e_{z}}{e_{z j}} \\
& E_{z, R o W}^{R}=e_{z} \sum_{z j \neq z} \frac{w_{z j}}{e_{z j}}
\end{aligned}
$$

$e_{z}=E_{z, R o W}^{R}$ only if $\sum_{z j \neq z}\left(w_{z j} / e_{z j}\right)=1$, and this cannot be true in general. However, absent changes in other exchange rates, proportional changes in a region $z$ 's exchange rate are equal to proportional changes in its multilateral real exchange rate.

\subsection{Calibration neutrality and consistency}

In this section, we examine the parametrization problem, first by determining the number of unknowns and constraints, and the corresponding number of degrees of freedom. Then we lay out the identification constraints that enable a solution, and we show that these are neutral, with special attention to the normalization rules in price $\times$ volume factoring. Finally, we show how the calibration sequence must be constructed carefully to ensure that calibration is consistent with whichever particular set of identification constraints is applied.

\subsubsection{Parametrization of Model 3}

Model 3 has $14 N+2 N(N-1)$ parameters, listed in Table 9. There are $4 N$ pairs $(=8 N)$ of free parameters: $\left(\sigma_{Z}, \rho_{Z}\right),\left(\sigma_{Z}^{M}, \rho_{Z}^{M}\right),\left(\tau_{Z}, \kappa_{Z}\right)$, and $\left(\tau_{Z}^{X}, \kappa_{Z}^{X}\right)$ which are set from exogenous sources. That leaves $6 N+2 N(N-1)$ parameters to be calibrated. The expanded version of Model 3 also has $13 N+1+3 N(N-1)$ variables whose benchmark values must be determined, and $10 N+3 N(N-1)$ equations. The SAM contains $N^{2}$ transaction flows. Table 10 summarizes the situation.

There are $N^{2}+7 N$ degrees of freedom, of which we dispose as follows: (1) three sets of prices are fixed exogenously: $e_{z}^{O}, P L_{z}^{*}$ and $P W_{z, z j}^{O} ;(2)$ we apply a normalization rule as an identification constraint to define the benchmark values of four volume variables: $E X T_{Z}^{O}, I M T_{Z}^{O}, Q_{Z}^{O}$ and $X S_{Z}^{O}$ (see below about price $\times$ volume factoring); (3) parameters $\alpha_{z j, z}^{M}$ and $\beta_{z, z j}^{X}$ are defined for each $z$ 
only up to a factor of proportionality, and we apply the $2 \mathrm{~N}$ normalization rules: $\sum_{z j} \beta_{z, z j}^{X}=1$ and $\sum_{z j} \alpha_{z j, z}^{M}=1$ (see Lemelin, Robichaud, Decaluwé \& Maisonnave, 2013, Appendices D4.3.2.2 and D4.3.3.2). Table 11 lists the identification constraints in the calibration process. The number of identification constraints is equal to the number of degrees of freedom in the calibration problem.

Table 9. Model 3 parameters

\begin{tabular}{|c|c|c|}
\hline & & Number \\
\hline$A_{Z}$ & $\begin{array}{l}\text { Scale parameter, Armington CES function between local production } \\
\text { and imports }\end{array}$ & $N$ \\
\hline$\alpha_{z}$ & $\begin{array}{l}\text { Share parameter, Armington CES function between local production } \\
\text { and imports }\end{array}$ & $N$ \\
\hline$\sigma_{z}$ & $\begin{array}{l}\text { Elasticity of substitution between local production and imports: } \\
0<\sigma_{Z}<\infty\end{array}$ & $N$ \\
\hline$\rho_{z}$ & Elasticity parameter: $\rho_{Z}=\frac{1-\sigma_{Z}}{\sigma_{z}}$, and $-1<\rho_{z}<\infty$ & $N$ \\
\hline$A_{Z}^{M}$ & $\begin{array}{l}\text { Scale parameter, Armington CES function between imports from } \\
\text { different regions }\end{array}$ & $N$ \\
\hline$\alpha_{z j, z}^{M}$ & $\begin{array}{l}\text { Share parameter, Armington CES function between imports from } \\
\text { different regions }\end{array}$ & $N(N-1)$ \\
\hline$\sigma_{z}^{M}$ & $\begin{array}{l}\text { Elasticity of substitution between imports from different regions: } \\
0<\sigma_{Z}^{M}<\infty\end{array}$ & $N$ \\
\hline$\rho_{Z}^{M}$ & Elasticity parameter: $\rho_{Z}^{M}=\frac{1-\sigma_{Z}^{M}}{\sigma_{Z}^{M}}$, and $-1<\rho_{Z}^{M}<\infty$ & $N$ \\
\hline$B_{Z}$ & Scale parameter, CET product aggregator & $N$ \\
\hline$\beta_{Z}$ & Share parameter, CET product aggregator & $N$ \\
\hline$\tau_{z}$ & Elasticity of transformation: $0<\tau_{z}<\infty$ & $N$ \\
\hline$\kappa_{Z}$ & Elasticity parameter: $\kappa_{Z}=\frac{\tau_{Z}+1}{\tau_{Z}}$, and $1<\kappa_{Z}<\infty$ & $N$ \\
\hline$B_{Z}^{X}$ & Scale parameter, CET exports aggregator & $N$ \\
\hline$\beta_{z, Z j}^{X}$ & Share parameter, CET exports aggregator & $N(N-1)$ \\
\hline$\tau_{Z}^{X}$ & Elasticity of transformation: $0<\tau_{Z}^{X}<\infty$ & $N$ \\
\hline$\kappa_{Z}^{X}$ & Elasticity parameter: $\kappa_{Z}^{X}=\frac{\tau_{Z}^{X}+1}{\tau_{Z}^{X}}$ and $1<\kappa_{Z}^{X}<\infty$ & $N$ \\
\hline
\end{tabular}


Table 10. Number of constraints and unknowns in the calibration process

\begin{tabular}{lll}
\hline$(1)$ & Parameters & $14 N+2 N(N-1)$ \\
$(2)$ & Minus: Free parameters & $8 N$ \\
$(3)=(1)-(2)$ & Equals: Parameters to be calibrated & $6 N+2 N(N-1)$ \\
$(4)$ & Plus: Variables & $13 N+1+3 N(N-1)$ \\
\hline$(5)=(3)+(4)$ & Number of unknowns & $19 N+1+5 N(N-1)$ \\
$(6)=(5)$ & Equal to & $5 N^{2}+14 N+1$ \\
\hline \hline$(7)$ & Number of equations & $10 N+3 N(N-1)$ \\
$(8)=(7)$ & Equal to & $3 N^{2}+7 N$ \\
$(9)$ & Walras' Law & 1 \\
$(10)$ & SAM transactions flows & $N^{2}$ \\
\hline$(11)=(8)+(9)+(10)$ & Number of constraints & $4 N^{2}+7 N+1$ \\
\hline \hline$(12)=(6)-(11)$ & Number of degrees of freedom & $N^{2}+7 N$ \\
\hline
\end{tabular}

Table 11. Identification constraints in the calibration process

$\begin{array}{ll}\text { (1) Exogenous prices } e_{Z}^{O}, P L_{Z}^{*} \text { and } P W_{Z, Z j}^{O} & 2 N+N(N-1) \\ \text { (2) }=(1) & N^{2}+N \\ \text { (3) Parameter normalization rules for } \alpha_{z j, Z}^{M} \text { and } \beta_{Z, Z j}^{X} & 2 N \\ \text { (4) Composite volume variable normalization rules for } E X T_{Z}^{O}, & 4 N \\ I M T_{Z}^{O}, Q_{Z}^{O} \text { and } X S_{Z}^{O} & \end{array}$

(5) Number of identification constraints $=(2)+(3)+(4) \quad N^{2}+7 N$

6.3.2 Price $\times$ volume factoring

One implication of the arbitrariness of the price $\times$ volume factoring mentioned in 2.3 relates to the calibration of CES and CET aggregates.22 Consider, for instance, the CET aggregate of exports to other regions.

Assume that $e_{z}, P W_{z, z j}$ and $E X_{z, z j}$ have already been assigned their benchmark values $e_{Z}^{O}, P W_{Z, Z j}^{O}$ and $E X_{z, Z j}^{O}$, and that free parameters $\tau_{Z}^{X}$ and $\boldsymbol{\kappa}_{Z}^{X}=\left(\tau_{Z}^{X}+1\right) / \tau_{Z}^{X}$ are defined. We want to assign benchmark values to $P X T_{Z}^{*}$ and $E X T_{Z}$, and calibrate parameters $B_{Z}^{X}$ and $\beta_{Z, z j}^{X}$ : we have $3 N+N(N-1)$ unknowns for $2 N+N(N-1)$ equations, namely equations (7), (8) and (14). That leaves $N$ degrees of freedom, which correspond to the arbitrariness of the price $\times$ volume

\footnotetext{
${ }^{22}$ The reader is referred to Appendices D4.3.2 and D4.3.3 in Lemelin, Robichaud, Decaluwé \& Maisonnave (2013) for a mathematical exposition of the calibration of CES and CET function parameters.
} 
factoring. Choosing a normalization rule eliminates the extra degrees of freedom. For instance, we can pose

$$
E X T_{Z}^{O}=\sum_{z j} E X_{Z, Z j}^{O}
$$

This is neutral (non-restrictive). Indeed, observe that, if $\operatorname{EXT}_{Z}^{O}, P X T_{Z}^{* O}$ and $B_{Z}^{X}$ satisfy equations (7), (8) and (14), then so do $E X T_{Z}^{O 2}=\lambda E X T_{Z}^{O}, P X T_{Z}^{* O 2}=P X T_{Z}^{* O} / \lambda$ and $B_{Z}^{X 2}=\lambda B_{Z}^{X}$, for any $\lambda>0$. Also note that the normalization rule (43) does not imply that exports to different regions are perfect substitutes. In simulations, the solution values will in general not satisfy equation (43). Alternatively, it would not be restrictive to fix $\operatorname{PXT}_{Z}^{* O}=1$ rather than using (43), provided $\operatorname{EXT}_{Z}^{O}$ is correctly calibrated according to (14), and the $B_{Z}^{X}$ are calibrated correspondingly (this is illustrated in the first calibration consistency test outlined in Appendix K.2, supplemental online material). ${ }^{23}$

\subsubsection{Calibration consistency and the calibration sequence}

So model parametrization requires that identification constraints such as (43), be imposed to solve the calibration problem. These identification constraints must be - and are - neutral. But also, the calibration sequence must be constructed carefully to ensure that calibration is consistent with whichever particular set of identification constraints is applied. This is illustrated in Table 12, which presents the calibration sequence of benchmark variables and parameters relating to exports.

Table 12 provides an example of the kind of pitfalls that can compromise calibration consistency. In view of steps 1, 3 and 6, it might be tempting to take a shortcut and replace step 7 with $\operatorname{PXT}_{Z}^{* O}=1$; that, however, would compromise calibration consistency, because the values assigned to $e_{z}^{O}$ and $P W_{z, z j}^{O}$ are arbitrary, and not necessarily 1 . Note in particular that adopting a different rule than the one in step 6 would change the benchmark value $X S_{Z}^{O}$ in step 8, then $P_{Z}^{* O}$ in step 9 , and it would modify the calibrated value of $B_{z}$ in step 11. So it is critical that these assignments be applied in the proper order. This is easy to verify here, but with a complex CGE model, the calibration procedure will be much more difficult to examine analytically.

\footnotetext{
${ }^{23}$ This is the choice made by McDonald and Thierfelder (2016, p.19).
} 
Because of the convenience of calibrating aggregates as in step 6, the calibration procedure of Model 3 applies the same rule to $E X T_{Z}^{O}, I M T_{Z}^{O}, Q_{Z}^{O}$ and $X S_{Z}^{O}$ (in that order). The calibration sequence of benchmark variables and parameters relating to imports is similar to that of exports. For a more complete presentation of the calibration procedure, the reader is referred to the GAMS code (supplemental online material), which is amply documented with comments in the code itself. A more in-depth presentation of the parametrization strategy, as it is applied to PEPw-1, can be found in Appendix E of Lemelin, Robichaud, Decaluwé \& Maisonnave (2013).

In a model as simple as Model 3, it is feasible to be reasonably certain of the consistency of the calibration procedure by diligent inspection of the code. In a complex model, even diligent inspection may overlook logical flaws, and testing offers an additional guarantee of consistency. To that end, the GAMS implementation of Model 3 presented in Appendix K (supplemental online material) contains options for the reader to test for calibration consistency; the options provided are examples of tests that could be performed on more elaborate models (and of course, no reader should resist the temptation to invent his/her own tests by modifying the programs provided). 
Journal of Global Economic Analysis, Volume 2 (2017), No. 1, pp. 215-324.

Table 12. Calibration sequence of export related benchmark variables and parameters

Step

1

2

3

4

5

6

7

8

9

$$
D_{Z}^{O}=\frac{\overline{P L_{Z}^{*} O} D_{Z}^{O}}{P L_{Z}^{* O}}=\frac{\text { SAM transactions flow }}{P L_{Z}^{* O}}
$$

$$
E X_{z, z j}^{O}=\frac{\overline{P W_{z, z j}^{O} E X_{z, z j}^{O}}}{P W_{z, z j}^{O}}=\frac{\text { SAM transactions flow }}{P W_{z, z j}^{O}}
$$

$$
E X T_{Z}^{O}=\sum_{z j} E X_{Z, Z j}^{O}
$$

$$
P X T_{Z}^{* O}=\frac{e_{Z}^{O} \sum_{z j} P W_{z, z j}^{O} E X_{z, z j}^{O}}{E X T_{Z}^{O}}
$$

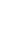

10

$$
\begin{gathered}
X S_{Z}^{O}=D_{Z}^{O}+E X T_{Z}^{O} \\
P_{Z}^{* O}=\frac{P L_{Z}^{*} D_{Z}^{O}+P X T_{Z}^{* O} E X T_{Z}^{O}}{X S_{Z}^{O}} \\
\beta_{Z}=\frac{P L_{Z}^{* O} D_{Z}^{O}{ }^{\left(1-\kappa_{z}\right)}}{\left(P L_{z}^{* O} D_{Z}^{O\left(1-\kappa_{z}\right)}+P X T_{Z}^{* O} E X T_{Z}^{O\left(1-\kappa_{z}\right)}\right)}
\end{gathered}
$$

continued... 
Table 12. Calibration sequence of export related benchmark variables and parameters (continued)

Step

Assignment

11

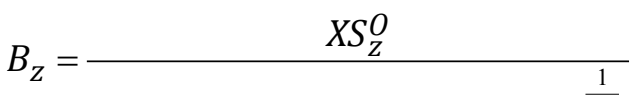

$$
\left[\beta_{Z}\left(D_{Z}^{O}\right)^{\kappa_{Z}}+\left(1-\beta_{Z}\right)\left(E X T_{Z}^{O}\right)^{\kappa_{Z}}\right]^{\kappa_{Z}}
$$

13

$$
\beta_{Z, z j}^{X}=\frac{P W_{z, z j}^{O}\left(E X_{Z, z j}^{O}\right)^{1-\tau_{Z}^{X}}}{\sum_{z j j} P W_{Z, z j j}^{O}\left(E X_{Z, z j j}^{O}\right)^{1-\tau_{Z}^{X}}}
$$

$$
B_{Z}^{X}=\frac{E X T_{Z}}{\left[\sum_{z j} \beta_{z, Z j}^{X}\left(E X_{Z, z j}\right)^{\kappa_{Z}^{X}}\right]^{\frac{1}{\kappa_{Z}^{X}}}}
$$

\section{Conclusion}

This paper was born out of the author's personal experience in critically examining a worldwide CGE model. At times, my PEP colleagues and I were confronted with pairs of model solutions that should be equivalent in theory, but didn't look like they were. In the course of (successfully) resolving such paradoxes, we feel we have gained a deeper understanding of the issues discussed here, and of CGE modeling in general. We want to share our insights, because the issues which we explore in this paper are seldom discussed explicitly in the descriptions of global multinational models. For that purpose, we develop a highly simplified skeleton model derived from the PEP-w-1 worldwide CGE model (Lemelin, Robichaud, Decaluwé \& Maisonnave, 2013), which nonetheless represents the essential structure of several world trade models.

The issues discussed may be grouped into four sets. First, in order to determine how many variables must be exogenously fixed in the closure, we delve into the identification of redundant equations. Beginning with a set of theoretical equations, the model is pared down by eliminating redundant equations, making sure in the process to avoid circular reasoning. The final step is to highlight Walras' Law. In all CGE models, one equilibrium relation is routinely discarded, and a numéraire is defined, invoking Walras' Law. But seldom is Walras' Law explicitly 
deducted from model equations as it is done in Appendix B (supplemental online material).

Secondly, we consider model closures, homogeneity with respect to prices, and the way the latter is dealt with in closing the model. We show that fixed nominal current account balances, a widely used closure rule, is in principle incorrect because it compromises model homogeneity (although it may be correct under certain conditions), and we formulate a theoretically correct fixed current account closure. Then, we broaden our view to see which variables other than $C A B X_{Z}$ could be made exogenous to close the model: we find that in the highly simplified skeleton model without exchange rates (Model 2), once the neoclassical fullemployment hypothesis is applied $\left(X S_{Z}=\overline{X S}_{Z}\right)$, there is really no other closure possible, except for variations on the numéraire.

Third, we introduce nominal exchange rates in the model (Model 3), and we show that they can be given arbitrary values. We go on to develop the idea that the supplementary degrees of freedom created by introducing exchange rates in the model allow for applying what we have labeled the FP closure (Fixed regional Price indexes), in which there is a fixed local numéraire for each region, and the endogenous nominal exchange rate is the price of the global numéraire good in terms of the regional one. We establish that the model under the FP closure is equivalent to the model under the fixed nominal exchange rates (FE) closure. Put otherwise, the two model closures are different in their implementation, but are mathematically and economically equivalent. In complex models, the FP closure facilitates the analysis of price changes: with regional numéraires, it is easier to distinguish between movements of regional prices relative to each other, and joint movements of regional prices relative to international prices, reflected in the exchange rates. Finally, although the FP nominal exchange rates are not real exchange rates, they are closely related: absent changes in other exchange rates, proportional changes in a region's exchange rate are equal to proportional changes in its multilateral real exchange rate.

The fourth and final set of questions examined relates to calibration neutrality and consistency. Even after so-called "free" parameters (elasticities and the like) have been specified, the calibration problem usually remains underdetermined. Consequently, identification constraints (arbitrary exogenous values and normalization rules) are imposed. Such identification constraints, given that they are based neither on observation (SAM flows), nor on theory (model equations), should be neutral, or non-restrictive. Moreover, whichever set of neutral identification constraints are used to make the calibration problem determinate, the calibration procedure should yield parametrized models that are equivalent. Model equivalence is defined in terms of the mathematical conditions which model solutions must meet for models to be considered equivalent. Finally, 
attention is drawn to the sequencing of calibration assignments and how incorrect sequencing can compromise neutrality and consistency.

The paper is complemented by a set of GAMS programs which implement the model, and allow for experimentation with alternate calibration procedures and model closures, and test for model homogeneity and calibration neutrality and consistency.

Nowadays, CGE models are routinely used to inform policy decisions. It is therefore critical that models be well conceived and that their results be correctly interpreted. But not all modelers and model-users have the leisure of reflecting on the foundations of their approach. This paper calls their attention to good modeling practice, and possible sources of errors and misinterpretations. Moreover, in the area of economic modeling, explicit and complete documentation is important for establishing a model's scientific basis, like the reproducibility of experiments is in other scientific domains. Black-box models are without scientific credibility. While experienced modelers usually seem to apply correct solutions to the issues raised in this paper, few actually discuss their choices. Perhaps some simply repeat standardized forms. Modelers are now challenged to explain why they do what they do the way they do it. They are challenged to move from oral to written transmission of proper modeling practice. In the end, it is hoped that the considerations raised here find their way into the curricula of CGE modeling training sessions.

\section{Acknowledgements}

I would like to thank my friends of the Partnership for Economic Policy (PEP) Research Network for creating the environment in which I was able to explore modelling issues. I also want to thank my referees for the outstanding quality of their in depth reviews: thanks to their contribution, this paper is much better than it would have been otherwise.

\section{References}

Bchir, M.H., Y. Decreux, J.-L. Guérin, and S. Jean. 2002a. “MIRAGE, a computable general equilibrium model for trade policy analysis." Paris: CEPII, Working Paper 2002-17. www.cepii.fr/CEPII/fr/publications/wp/ abstract.asp?NoDoc $=958$

Bchir, M.H., Y. Decreux, J.-L. Guérin, and S. Jean. 2002b. "MIRAGE, un modèle d'équilibre général calculable pour l'évaluation des politiques commerciales". Economie internationale, 89-90: 109-153. 
Chinn, M.D. 2006. "A primer on real effective exchange rates: Determinants, overvaluation, trade flows and competitive devaluation". Open economies review, 17(1): 115-143. ${ }^{24}$ doi: $10.1007 /$ s11079-006-5215-0

Chinn, M.D. 2008. Real Exchange Rates, in S.N. Durlauf and L.E. Blume (eds.), The New Palgrave Dictionary of Economics, Second edition, Palgrave Macmillan. ${ }^{25}$

Decaluwé, B., A. Martens, and L. Savard. 2001. La politique économique du développement et les modèles d'équilibre général calculable. Montréal : Presses de l'Université de Montréal.

Decaluwé, B., A. Martens, and M. Monette. 1988. "Macroclosures in Open Economy CGE Models: A Numerical Appraisal". International Journal of Development Planning Literature, 3, 69-90.26

Decreux, Y. and H. Valin. 2007. "MIRAGE, Updated Version of the Model for Trade Policy Analysis with a Focus on Agriculture and Dynamics." Paris: CEPII, Working Paper no. 2007-15. cepii.fr/CEPII/fr/publications/wp/ abstract.asp?NoDoc $=958$

Delpiazzo, E. 2010. “The mozambican participation in SADC. A liberalization process through different models and different closures." Ph.D. thesis, Universitá Cattolica del Sacro Cuore, Milano, Italy. tesionline.unicatt.it/handle/10280/1109

Dewatripont, M., and G. Michel. 1987. "On Closure Rules, Homogeneity and Dynamics in Applied General Equilibrium Models". Journal of Development Economics, 26(1), 65-76. doi: 10.1016/0304-3878(87)90052-6

Hertel, T.W. 1997. Global Trade Analysis: Modeling and Applications, Cambridge: Cambridge University Press.

Hübler, M. 2011. "Technology diffusion under contraction and convergence: A CGE analysis of China". Energy Economics. 33(1): 131-142. doi: 10.1016/j.eneco.2010.09.002

Ianchovichina, E., R. McDougall. 2001. “Theoretical Structure of Dynamic GTAP, Edition 1.1." Center for Global Trade Analysis, GTAP Technical Paper No. 17. gtap.agecon.purdue.edu/models/Dynamic/model.asp

Ianchovichina, E., R. McDougall, and T. Hertel. 2000. "A Disequilibrium Model of International Capital Mobility." Center for Global Trade Analysis, GTAP Working Paper No. 10.gtap.agecon.purdue.edu/models/Dynamic/model.asp

${ }^{24}$ A preliminary version of this article is downloadable from http:/ / www.ssc.wisc.edu/ mchinn/primer_OER.pdf (accessed 2017-02-15).

${ }^{25}$ A preliminary version of this article is downloadable from http://www.ssc.wisc.edu/ mchinn/NewPalgrave_RER.pdf (accessed 2017-02-15).

${ }^{26}$ A preliminary version of this paper is available under the title "Macroclosures in Open Economy CGE Models: A Numerical Reappraisal". Montreal: CRDE, Université de Montreal, Cahier 8704. papyrus.bib.umontreal.ca/xmlui/handle/1866/417 (accessed 2015-09-24) 
Lemelin, A., and V. Robichaud. 2014. "PEP-w-t - The PEP standard multi-region, recursive dynamic world CGE model: Update, with a reference scenario." Partnership for Economic Policy (PEP) Research Network, Université Laval, Québec. pep-net.org/pep-standard-cge-models

Lemelin, A., V. Robichaud, and B. Decaluwé. 2013. "Endogenous current account balances in a world CGE model with international financial assets". Economic Modelling, 32(2): 146-160. doi: 10.1016/j.econmod.2013.01.046

Lemelin, A., V. Robichaud, and B. Decaluwé. 2014. "PEP-w-t-fin: The PEP standard multi-region, recursive dynamic world CGE model with bilateral financial assets." Partnership for Economic Policy (PEP) Research Network, Université Laval, Québec. pep-net.org/pep-standard-cge-models

Lemelin, A., V. Robichaud, B. Decaluwé, and H. Maisonnave. 2013. "PEP-w-1: The PEP standard multi-region, single-period world CGE model, Version 4.0." Partnership for Economic Policy (PEP) Research Network, Université Laval, Québec. pep-net.org/pep-standard-cge-models

Lofgren, H., R. Lee Harris, and S. Robinson. 2002. "A Standard Computable General Equilibrium (CGE) Model in GAMS." Microcomputers in policy research 5, International Food Policy Research Institute, Washington, D.C. ifpri.org/publication/standard-computable-general-equilibrium-cge-modelgams-0

McDonald, S., and K. Thierfelder. 2016. "Globe v2: A SAM based global CGE model using GTAP data." Applied General Equilibrium Modelling. cgemod.org.uk/globe2.html

McKibbin, W.J., and A. Stoeckel. 2009. "Modelling the global financial crisis". Oxford Review of Economic Policy. 25(4): 581-607. doi: 10.1093/oxrep/grq012

McKibbin, W.J., and P.J. Wilcoxen. 1999. "The theoretical and empirical structure of the G-Cubed model". Economic Modelling, 16(1): 123-148. doi: 10.1016/S02649993(98)00035-2

Rattsø, J. 1982. “Different Macroclosures of the Original Johansen Model and Their Impact on Policy Evaluation". Journal of Policy Modeling, 4(1): 85-97. doi: 10.1016/0161-8938(82)90006-0

Sen, A. 1963. "Neo-Classical and Neo-Keynesian Theories of Distribution". The Economic Record, 39(85): 53-64. doi: 10.1111/j.1475-4932.1963.tb01459.x

Springer, K. 2003. Climate policy in a globalizing world. A CGE model with capital mobility and trade. Kiel Studies Vol. 320. Berlin: Springer.

van der Mensbrugghe, D. 2010. "The ENVironmental Impact and Sustainability Applied General Equilibrium (ENVISAGE) Model, Version 7.1." Washington, D.C.: World Bank. siteresources.worldbank.org/INTPROSPECTS/Resources/ 334934-1314986341738/Env7_1Jan10b.pdf 


\section{Appendix A: Detailed statement of Model 1}

\section{Production}

With fixed production factors and full employment, output is fixed in each region $z: X S_{Z}=\overline{X S}_{Z}$, where $X S_{Z}$ is domestic production in region $z$. That constraint, however, is not part of the model; rather, it is a closure equation.

Income and savings

Regional income is equal to the value of production.

$$
Y_{Z}=P_{Z} X S_{Z}
$$

where $P_{Z}$ is the producer price in region $\mathrm{z}$ and $Y_{z}$ is the income in region $\mathrm{z}$.

The model is static, so we can define "consumption" to include both current consumption and investment. It follows that savings, the difference between income and consumption, are equal to the current account balance (CAB). So the regional agent's budget constraint is

$$
C A B_{Z}=Y_{Z}-P C_{Z} Q_{Z}
$$

where $C A B_{z}$ is the current account balance of region $z ; P C_{z}$ is the price of the composite good in region $z ; Q_{Z}$ is domestic demand for the composite good in region $z$.

To make the model more compact, we substitute (A.1) into (A.2), which becomes ${ }^{27}$

$$
C A B_{Z}=P_{Z} X S_{Z}-P C_{Z} Q_{Z}
$$

We can eliminate equation (A.1) and variable $Y_{Z}$ from the model.

Not only is the current account balance equal to savings, but it is by definition equal to the difference between the aggregate value of exports and the aggregate value of imports.

$$
C A B_{Z}=P X T_{Z} E X T_{Z}-P M T_{Z} I M T_{Z}
$$

where $I M T_{Z}$ is total imports of region $z ; E X T_{Z}$ is total exports of region $z ; P M T_{Z}$ is the price of composite imports in region $z ; P X T_{Z}$ is the price of composite exports of region $z$.

\footnotetext{
${ }^{27}$ Equations that appear in the main text are numbered as they are in the main text.
} 
Trade

Production is allocated between sales on the domestic market and exports so as to maximize its value subject to a CET transformation function.

$$
X S_{Z}=B_{z}\left[\beta_{z} D_{z}^{\kappa_{z}}+\left(1-\beta_{z}\right) E X T_{Z}{ }^{\kappa_{z}}\right]^{\frac{1}{\kappa_{z}}}
$$

where $\kappa_{z}=\frac{\tau_{z}+1}{\tau_{z}}$, with $0<\tau_{z}<\infty$, and

$$
\frac{E X T_{Z}}{D_{z}}=\left(\frac{\beta_{z}}{1-\beta_{z}} \frac{P X T_{Z}}{P L_{z}}\right)^{\tau_{Z}}
$$

where $D_{z}$ is domestic demand for the locally produced good in region $z ; P L_{z}$ is the market price of local product in region $z$; and $B_{z}$ is a scale parameter and $\beta_{z}$ is the share parameter of the CET product aggregator; $\tau_{z}$ is the elasticity of transformation: $0<\tau_{z}<\infty$

$\kappa_{z}=\frac{\tau_{z}+1}{\tau_{z}}: 1<\kappa_{z}<\infty$

Total exports are allocated among destination regions so as to maximize their value subject to a CET transformation function.

$$
E X T_{Z}=B_{Z}^{X}\left[\sum_{z j} \beta_{Z, Z j}^{X}\left(E X_{Z, Z j}\right)^{\kappa_{Z}^{X}}\right]^{\frac{1}{\kappa_{Z}^{X}}}
$$

where $\kappa_{Z}^{X}=\frac{\tau_{Z}^{X}+1}{\tau_{Z}^{X}}$, with $0<\tau_{Z}^{X}<\infty$, and

$$
E X_{Z, Z j}=\frac{E X T_{Z}}{\left(B_{Z}^{X}\right)^{1+\tau_{Z}^{X}}}\left[\frac{P W_{z, Z j}}{\beta_{Z, Z j}^{X} P X T_{Z}}\right]^{\tau_{Z}^{X}}
$$

where $B_{Z}^{X}$ is the scale parameter and $\beta_{Z, Z j}^{X}$ is the share parameter of the CET exports aggregator; $\tau_{Z}^{X}$ is the elasticity of transformation, with $0<\tau_{Z}^{X}<\infty$; $\kappa_{Z}^{X}=\frac{\tau_{Z}^{X}+1}{\tau_{Z}^{X}}$ is the elasticity parameter, with $1<\kappa_{Z}^{X}<\infty ; P W_{Z, Z j}$ is the world 
price of exports from region $z$ to region $z j$; and $E X_{z, z j}$ are exports by region $z$ to region $z j$.

Under the Armington hypothesis, domestic demand is distributed between the domestically produced good and imports so as to maximize the quantity acquired, subject to a CES aggregator function.

$$
Q_{Z}=A_{z}\left[\alpha_{Z} D_{z}^{-\rho_{z}}+\left(1-\alpha_{z}\right) I M T_{z}^{-\rho_{z}}\right]^{-\frac{1}{\rho_{z}}}
$$

where $\rho_{z}=\frac{1-\sigma_{z}}{\sigma_{z}}$, with $0<\sigma_{z}<\infty$, and

$$
\frac{I M T_{z}}{D_{z}}=\left(\frac{1-\alpha_{z}}{\alpha_{z}} \frac{P L_{z}}{P M T_{Z}}\right)^{\sigma_{z}}
$$

where $A_{Z}$ is the scale parameter, and $\alpha_{z}$ the share parameter of the Armington CES function between local production and imports; $\sigma_{z}$ is the elasticity of substitution between local production and imports, with $0<\sigma_{z}<\infty$; and $\rho_{z}=\frac{1-\sigma_{z}}{\sigma_{z}}$ is the elasticity parameter, with $-1<\rho_{z}<\infty$.

Under the Armington hypothesis, imports are distributed among exporting regions so as to maximize the quantity acquired, subject to a CES aggregator function.

$$
I M T_{Z}=A_{Z}^{M}\left[\sum_{z j} \alpha_{z j, z}^{M} I M_{z j, z}^{-\rho_{Z}^{M}}\right]^{\frac{-1}{\rho_{Z}^{M}}}
$$

where $\rho_{Z}^{M}=\frac{1-\sigma_{Z}^{M}}{\sigma_{Z}^{M}}$, with $0<\sigma_{Z}^{M}<\infty$, and

$$
I M_{z j, z}=\frac{I M T_{Z}}{\left(A_{z}^{M}\right)^{1-\sigma_{Z}^{M}}}\left[\frac{\alpha_{z j, z}^{M} P M T_{Z}}{P W_{z j, z}}\right]^{\sigma_{Z}^{M}}
$$

where $A_{z}^{M}$ is the scale parameter, and $\alpha_{z j, z}^{M}$ the share parameter of the Armington CES function between imports from different regions; $\sigma_{Z}^{M}$ is the 
elasticity of substitution between imports from different regions, with $0<\sigma_{Z}^{M}<\infty$; and $\rho_{Z}^{M}=\frac{1-\sigma_{Z}^{M}}{\sigma_{Z}^{M}}$ is the elasticity parameter, with $-1<\rho_{Z}^{M}<\infty$.

Prices

The value of production is equal to the sum of the value of sales on the domestic market and exports.

$$
P_{Z} X S_{Z}=P L_{Z} D_{z}+P X T_{Z} E X T_{Z}
$$

The total value of exports is equal to the sum of values of exports to all regions

$$
P X T_{Z} E X T_{Z}=\sum_{z j} P W_{z, z j} E X_{z, z j}
$$

Total expenditures are equal to the sum of purchases on the domestic market and the value of imports.

$$
P C_{Z} Q_{Z}=P L_{z} D_{Z}+P M T_{Z} I M T_{Z}
$$

The total value of imorts is equal to the sum of values of imports from all regions

$$
P M T_{Z} I M T_{Z}=\sum_{z j} P W_{z j, z} I M_{z j, z}
$$

\section{Equilibrium}

Imports from region $z j$ by region $z$ must be equal to exports from region $z j$ to region $z$.

$$
E X_{z j, z}=I M_{z j, z}
$$

The world sum of current account balances, expressed in the international currency, must be zero.

$$
\sum_{Z} C A B_{Z}=0
$$




\section{Appendix B: Redundant equations in model 1}

We now show that

- equations (7) and (8) together imply (14), which is therefore redundant;

- equations (11) and (12) together imply (16), which is therefore redundant;

- equations (4) and (17) together imply (18), which is therefore redundant;

- equations (13), (15) and (3) together imply (4), which is therefore redundant;

- equations (14) (or equivalently the combination of (7) and (8)),

(16) (or equivalently the combination of (11) and (12)),

(13), (15) and (3), and

(18) (or alternatively the combination of (13), (15) and (3),

which together imply (4), and equations (4) and (17))

together imply that, if equation (3) is satisfied for $N-1$ regions, then it is also satisfied for the $N^{\text {th }}$ one (Walras' Law). Therefore, one equation of the set (3) may be discarded as redundant. 


\section{B.1 Redundancy of equations (14) and (16)}

Given (7) and (8), equation (14) is redundant, and given (11) and (12), equation (16) is redundant. This is demonstrated in parallel in three steps.

Step 1: Substitute (8) into (7), and (12) into (11), and develop.

$$
\begin{aligned}
& E X T_{Z}=B_{Z}^{X}\left[\sum_{z j} \beta_{z, z j}^{X}\left(\frac{E X T_{Z}}{\left(B_{Z}^{X}\right)^{1+\tau_{Z}^{X}}}\left[\frac{P W_{z, z j}}{\beta_{z, z j}^{X} P X T_{z}}\right]^{\tau_{Z}^{X}}\right]^{\kappa_{Z}^{X}}\right]^{\frac{1}{\kappa_{Z}^{X}}} \quad I M T_{z}=A_{z}^{M}\left[\sum_{z j} \alpha_{z j, z}^{M}\left(\frac{I M T_{z}}{\left(A_{Z}^{M}\right)^{1-\sigma_{Z}^{M}}}\left[\frac{\alpha_{z j, z}^{M} P M T_{z}}{P W_{z j, z}}\right]^{\sigma M}\right]^{-\rho_{z}^{M}}\right]^{\frac{-1}{\rho_{z}^{M}}} \\
& E X T_{Z}=B_{Z}^{X}\left[\sum_{z j} \beta_{z, z j}^{X}\left(\frac{E X T_{Z}}{\left(B_{Z}^{X}\right)^{1+\tau \tau_{Z}^{X}}}\left[\frac{P W_{z, z j}}{\beta_{z, z j}^{X} P X T_{z}}\right]^{\tau_{Z}^{X}}\right]^{\frac{\tau_{z}^{X}+1}{\tau_{Z}^{X}}}\right]^{\frac{\tau_{Z}^{X}}{\tau_{Z}+1}}
\end{aligned}
$$

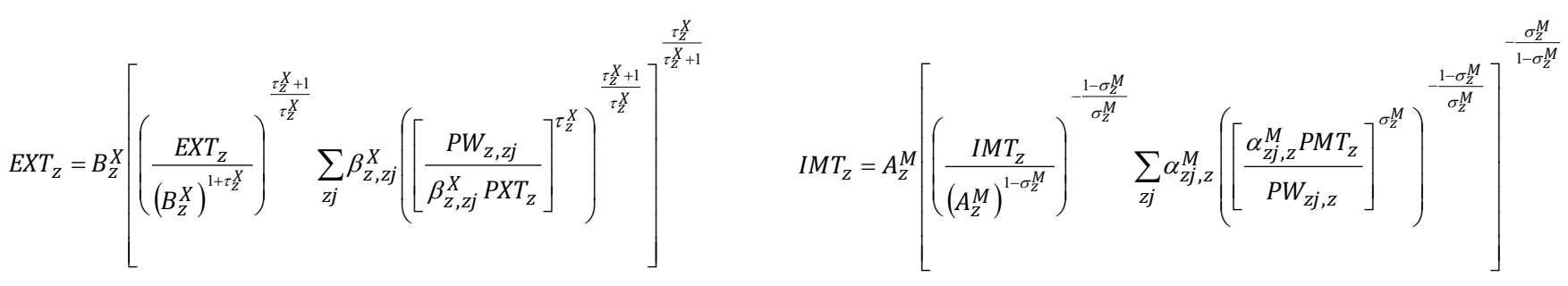




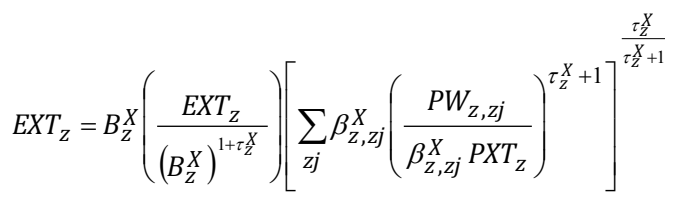

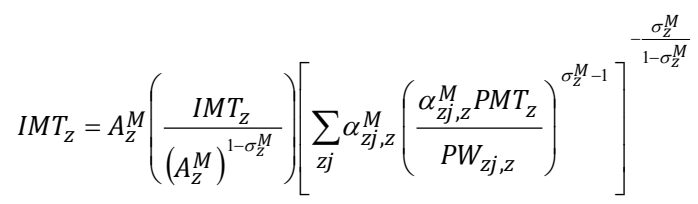

$$
\begin{aligned}
& 1=\frac{1}{\left(B_{Z}^{X}\right)^{\tau_{Z}^{X}}}\left[\sum_{z j} \beta_{Z, z j}^{X}\left(\frac{P W_{z, z j}}{\beta_{z, z j}^{X} P X T_{Z}}\right)^{\tau_{Z}^{X}+1}\right]^{\frac{\tau_{\frac{X}{X}}}{\tau_{Z}+1}}
\end{aligned}
$$

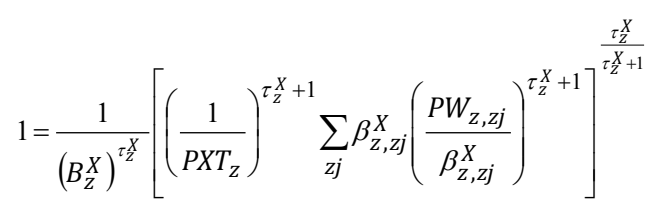

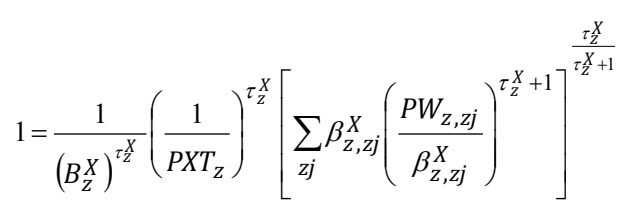

$$
\begin{aligned}
& \left(P X T_{Z}\right)^{\frac{\tau X}{Z}}=\frac{1}{\left(B_{Z}^{X}\right)^{\tau \frac{Z Z}{Z}}}\left[\sum_{z j} \beta_{Z, z j}^{X}\left(\frac{P W_{z, z j}}{\beta_{Z, z j}^{X}}\right)^{\tau_{Z}^{X}+1}\right]^{\frac{\tau_{Z}^{X}}{z_{Z}^{Z}+1}} \\
& \begin{array}{l}
1=\frac{1}{\left(A_{z}^{M}\right)^{-\sigma_{z}^{M}}}\left[\sum_{z j} \alpha_{z j, z}^{M}\left(\frac{P W_{z j, z}}{\alpha_{z j, z}^{M} P M T_{z}}\right)^{1-\sigma_{z}^{M}}\right]^{\frac{-\sigma^{M}}{1-\sigma_{z}^{M}}} \\
=\frac{1}{\left(A_{z}^{M}\right)^{-\sigma z_{z}^{M}}}\left[\left(\frac{1}{P M T_{z}}\right)^{1-\sigma_{z}^{M}} \sum_{z j} \alpha_{z j, z}^{M}\left(\frac{P W_{z j, z}}{\alpha_{z j, z}^{M}}\right)^{1-\sigma_{z}^{M}}\right]^{-\frac{\sigma_{z}^{M}}{1-\sigma_{z}^{M}}}
\end{array} \\
& 1=\frac{1}{\left(A_{z}^{M}\right)^{-\sigma_{z}^{M}}}\left(\frac{1}{P M T_{z}}\right)^{\sigma_{z}^{M}}\left[\sum_{z j} \alpha_{z, z, z}^{M}\left(\frac{P W_{z, z, z}}{\alpha_{z, z}^{M}}\right)^{1-\sigma_{z}^{M}}\right]^{-\frac{\sigma_{z}^{M}}{1-\sigma_{z}^{M}}} \\
& \left(P M T_{z}\right)^{-\sigma_{z}^{M}}=\frac{1}{\left(A_{z}^{M}\right)^{-\sigma_{z}^{M}}}\left[\sum_{z j} \alpha_{z j, z}^{M}\left(\frac{P W_{z j, z}}{\alpha_{z, z, z}^{M}}\right)^{1-\sigma_{z}^{M}}\right]^{-\frac{\sigma_{z}^{M}}{1-\sigma_{z}^{M}}}
\end{aligned}
$$




$$
\begin{array}{rr}
P X T_{Z}=\frac{1}{B_{Z}^{X}}\left[\sum_{z j} \beta_{z, z j}^{X}\left(\frac{P W_{z, z j}}{\beta_{z, z j}^{X}}\right)^{\tau_{Z}^{X}+1}\right]^{\frac{1}{z_{Z}^{X}+1}} & P M T_{z}=A_{Z}^{M}\left[\sum_{z j} \alpha_{z j, z}^{M}\left(\frac{P W_{z j, z}}{\alpha_{z j, z}^{M}}\right)^{1-\sigma \sigma_{z}^{M}}\right]^{\frac{1}{1-\sigma_{z}^{M}}} \\
P X T_{z}=\frac{1}{B_{Z}^{X}}\left[\sum_{z j}\left(\frac{1}{\beta_{z, z j}^{X}}\right)^{\tau_{z}^{X}}\left(P W_{z, z j}\right)^{\frac{1}{\tau_{Z}^{X}+1}}\right]^{\frac{1}{\tau_{Z}^{X}+1}} & P M T_{z}=A_{Z}^{M}\left[\sum_{z j}\left(\alpha_{z j, z}^{M}\right)^{\sigma_{z}^{M}}\left(P W_{z j, z}\right)^{1-\sigma \sigma_{z}^{M}}\right]^{\frac{1}{1-\sigma_{z}^{M}}}
\end{array}
$$

Step 2: Multiply both sides of (8) by $P W_{z, z j}$, and both sides of (12) by $P W_{z j, z}$, and sum over $z j$.

$$
\begin{aligned}
& \sum_{z j} P W_{z, z j} E X_{z, z j}=\sum_{z j} P W_{z, z j} \frac{E X T_{Z}}{\left(B_{Z}^{X}\right)^{1+\tau Z Z}}\left[\frac{P W_{z, z j}}{\beta_{z, z j}^{X} P X T_{z}}\right]^{\tau_{Z}^{X}} \quad \sum_{z j} P W_{z j, z} I M_{z j, z}=\sum_{z j} P W_{z j, z} \frac{I M T_{Z}}{\left(A_{z}^{M}\right)^{1-\sigma_{Z}^{M}}}\left[\frac{\alpha_{z j, z}^{M} P M T_{z}}{P W_{z j, z}}\right]^{\sigma_{Z}^{M}}
\end{aligned}
$$

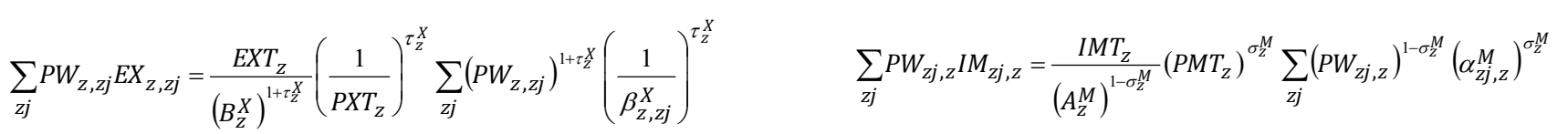

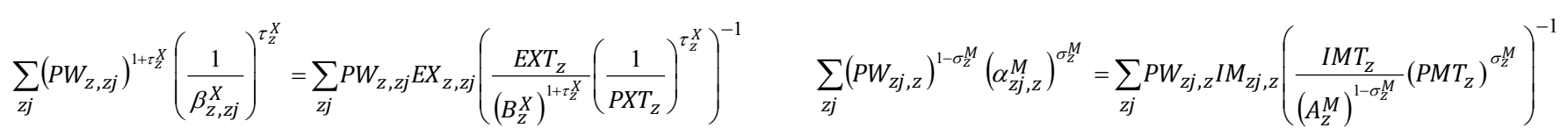

Step 3: Substitute the right-hand side of the last equation in Step 2 into the last equation in Step 1

$$
P X T_{Z}=\frac{1}{B_{Z}^{X}}\left[\sum_{z j} P W_{z, z j} E X_{z, z j}\left(\frac{E X T_{Z}}{\left(B_{Z}^{X}\right)^{1+\tau_{Z}^{X}}}\left(\frac{1}{P X T_{Z}}\right)^{\tau_{Z}^{X}}\right)^{-1}\right]^{\frac{1}{\tau_{Z}^{X}+1}} \quad P M T_{Z}=A_{Z}^{M}\left[\sum_{z j} P W_{z j, z} I M_{z j, z}\left(\frac{I M T_{Z}}{\left(A_{Z}^{M}\right)^{1-\sigma_{Z}^{M}}}\left(P M T_{Z}\right)^{\sigma_{Z}^{M}}\right)^{-1}\right]^{\frac{1}{1-\sigma_{Z}^{M}}}
$$




$$
\begin{aligned}
& P X T_{Z}=\frac{1}{B_{Z}^{X}}\left(\frac{E X T_{Z}}{\left(B_{Z}^{X}\right)^{1+t z \frac{X}{Z}}}\left(\frac{1}{P X T_{Z}}\right)^{\tau_{Z}^{X}}\right)^{-\frac{1}{\tau_{Z}^{X}+1}}\left[\sum_{z j} P W_{z, z j} E X_{z, z j}\right]^{\frac{1}{\tau_{Z}^{X}+1}}
\end{aligned}
$$

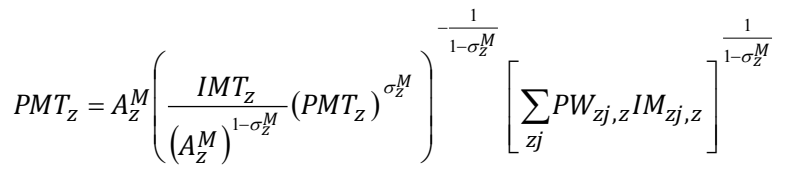

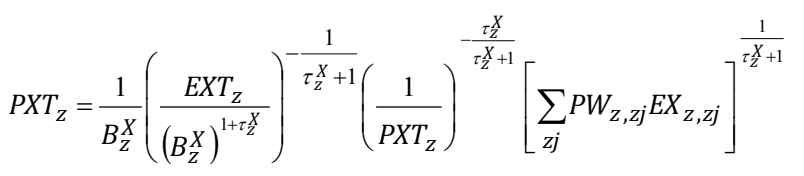

$$
\begin{aligned}
& P M T_{z}=A_{z}^{M}\left(\frac{I M T_{z}}{\left(A_{z}^{M}\right)^{1-\sigma_{z}^{M}}}\right)^{\frac{1}{1-\sigma_{z}^{M}}}\left(\frac{1}{P M T_{z}}\right)^{\frac{\sigma_{z}^{M}}{1-\sigma z^{M}}}\left[\sum_{z j} P W_{z j, z} I M_{z j, z}\right]^{\frac{1}{1-\sigma \sigma_{z}^{M}}} \\
& \left(P X T_{Z} E X T_{Z}\right)^{\frac{1}{z_{Z}+1}}=\left[\sum_{z j} P W_{Z, z j} E X_{Z, z j}\right]^{\frac{1}{z_{z}+1}} \\
& \left(P M T_{z} I M T_{z}\right)^{\frac{1}{1-\sigma z_{z}^{M}}}=\left[\sum_{z j} P W_{z j, z} I M_{z j, z}\right]^{\frac{1}{1-\sigma z_{z}^{M}}} \\
& P X T_{z} E X T_{z}=\sum_{z j} P W_{z, z j} E X_{z, z j} \\
& P M T_{z} I M T_{z}=\sum_{z j} P W_{z j, z} I M_{z j, z}
\end{aligned}
$$

The final equations in Step 3 are identical to (14) and (16), which are therefore redundant. 


\section{B.2 Equation (18) redundant}

Substitute (14) and (16) into (4) and sum over $z$.

$$
\begin{aligned}
& \sum_{Z} C A B_{Z}=\sum_{z} \sum_{z j} P W_{z, z j} E X_{z, z j}-\sum_{z} \sum_{z j} P W_{z j, z} I M_{z j, z} \\
& \sum_{z} C A B_{z}=\sum_{z} \sum_{z j} P W_{z, z j} E X_{z, z j}-\sum_{z} \sum_{z j} P W_{z, z j} I M_{z, z j}
\end{aligned}
$$

Note the change in the order of indices between (B.1) and (B.2).

$$
\sum_{z} C A B_{Z}=\sum_{z} \sum_{z j} P W_{z, z j}\left(E X_{Z, z j}-I M_{Z, z j}\right)
$$

And, given (17), we have (18). Equations (4) and (17) together imply (18), which is therefore redundant.

\section{B.3 Equation (4) redundant}

Substitute (13) and (15) into (3) to obtain (4). Since equations (13), (15) and (3) together imply (4), the latter is therefore redundant.

\section{B.4 Walras' Law}

We shall now examine how Walras' Law applies to our model. Define excess demands on the domestic and international markets respectively as

$$
\begin{gathered}
X D_{z}=D_{z}^{D}-D_{z}^{O} \\
X M_{z j, z}=I M_{z j, z}-E X_{z j, z}
\end{gathered}
$$

where $D_{Z}^{D}$ is the demand for domestic production, and $D_{Z}^{O}$ is supply to the domestic market.

It is shown in Appendix $\mathrm{D}$ that the demand for domestic production as a function of relative prices and the demand for the composite good is

$$
D_{Z}^{D}=\left(A_{Z}\right)^{\sigma_{Z}-1}\left(\frac{\alpha_{Z} P C_{Z}}{P L_{Z}}\right)^{\sigma_{z}} Q_{Z}
$$

Similarly, it is shown in Appendix E that supply to the domestic market as a function of relative prices and total production is

$$
D_{Z}^{O}=\left(\frac{1}{B_{Z}}\right)^{\tau_{Z}+1}\left(\frac{P L_{Z}}{\beta_{Z} P_{Z}}\right)^{\tau_{Z}} X S_{Z}
$$


In Appendix F, it is shown that region $z^{\prime}$ s demand for imports from $z j j$, as a function of relative prices and demand for the composite good, is given by

$$
I M_{z j j, z}=\left(\frac{1}{A_{z}^{M}}\right)^{1-\sigma_{z}^{M}}\left(\frac{P W_{z j j, z}}{\alpha_{z j j, z}^{M} P M T_{z}}\right)^{-\sigma_{z}^{M}}\left(A_{z}\right)^{\sigma_{z}-1}\left(\frac{\left(1-\alpha_{z}\right) P C_{z}}{P M T_{z}}\right)^{\sigma_{z}} Q_{z}
$$

Finally, it is shown in Appendix G that region $z$ 's supply of exports towards $z j j$, as a function of relative prices and total supply, is

$$
E X_{Z, z j j}=\left(\frac{1}{B_{Z}^{X}}\right)^{\tau_{Z}^{X}+1}\left(\frac{P W_{z, z j j}}{\beta_{Z, z j j}^{X} P X T_{Z}}\right)^{\tau_{Z}^{X}}\left(\frac{1}{B_{z}}\right)^{\tau_{z}+1}\left(\frac{P X T_{z}}{\left(1-\beta_{z}\right) P_{Z}}\right)^{\tau_{z}} X S_{Z}
$$

Supply and demand equations (B.24)-(B.27) are homogeneous with respect to prices. Their mathematical derivations are given in Appendices D-G (look for equations (D.43), (E.46), (F.32) and (G.35)).

The value of excess demand on the domestic and international markets is given by

$$
\begin{gathered}
P L_{z} X D_{z}=P L_{z} D_{z}^{D}-P L_{z} D_{z}^{O} \\
P W_{z j, z} X M_{z j, z}=P W_{z j, z} I M_{z j, z}-P W_{z j, z} E X_{z j, z}
\end{gathered}
$$

The aggregate value of all excess demands, expressed in terms of the international currency, is

$$
\begin{aligned}
& \sum_{z} P L_{z} X D_{z}+\sum_{z} \sum_{z j} P W_{z, z j} X M_{z, z j}=\sum_{z} P L_{z} D_{Z}^{D}- \\
& \sum_{z} P L_{z} D_{z}^{O}+\sum_{z} \sum_{z j} P W_{z, z j} I M_{z, z j}-\sum_{z} \sum_{z j} P W_{z, z j} E X_{z, z j}
\end{aligned}
$$

Substitute equations (14) and (16) into the last equation. It is true that equations (14) and (16) are no longer present among the model equations (they have already been discarded as redundant), but they are implied ${ }^{27}$ :

- $\quad$ equations (7) and (8) together imply (14) (see above and Appendix B);

- equations (11) and (12) together imply (16) (see above and Appendix B).

\footnotetext{
27 We underline this as a precaution against the logical pitfall that would consist in using a discarded equation that could no longer be considered implicit in the model because the equations which imply it would also be absent from the model.
} 
The substitution yields

$$
\begin{aligned}
& \sum_{z} P L_{z} X D_{z}+\sum_{z} \sum_{z j} P W_{z, z j} X M_{z, z j}= \\
& \sum_{z} P L_{z} D_{Z}^{D}-\sum_{z} P L_{z} D_{Z}^{O}+\sum_{z j} P M T_{z j} I M T_{z j}-\sum_{z} P X T_{Z} E X T_{Z} \\
& \sum_{z} P L_{z} X D_{z}+\sum_{z} \sum_{z j} P W_{z, z j} X M_{z, z j}= \\
& \sum_{Z} P L_{z} D_{Z}^{D}-\sum_{z} P L_{z} D_{Z}^{O}+\sum_{Z} P M T_{Z} I M T_{Z}-\sum_{Z} P X T_{Z} E X T_{Z} \\
& \sum_{z} P L_{z} X D_{z}+\sum_{z} \sum_{z j} P W_{z, z j} X M_{z, z j}= \\
& \sum_{Z}\left[\left(P L_{Z} D_{Z}^{D}+P M T_{Z} I M T_{Z}\right)-\left(\sum_{Z} P L_{Z} D_{Z}^{O}+\sum_{Z} P X T_{Z} E X T_{Z}\right)\right] \\
& \sum_{Z} P L_{z} X D_{z}+\sum_{z} \sum_{z j} P W_{z, z j} X M_{z, z j}= \\
& \sum_{Z}\left[\left(P L_{Z} D_{Z}^{D}+P M T_{Z} I M T_{Z}\right)-\left(\sum_{z} P L_{Z} D_{Z}^{O}+\sum_{Z} P X T_{Z} E X T_{Z}\right)\right]
\end{aligned}
$$

Substitute (13) and (15) into the last equation, and

$$
\sum_{Z} P L_{Z} X D_{Z}+\sum_{z} \sum_{z j} P W_{Z, z j} X M_{Z, Z j}=\sum_{Z}\left(P C_{Z} Q_{Z}-P_{Z} X S_{Z}\right)
$$

where the regional agents' budget constraints are given by (3).

Regional budget constraints are homogeneous with respect to prices and nominal values ${ }^{28}$. Substitute the regional budget constraints into (B.35) to obtain

\footnotetext{
${ }^{28}$ In the traditional development of Walras' Law, the income and expenditures of each agent are constained to be equal, so that their budget constraints are homogeneous with respect to prices. Here, agents may have surpluses or deficits (non-zero CABs), the counterpart of which are, broadly speaking, international "loans". It follows that homogeneity must be defined not with respect to prices only, but with respect to prices and nominal values.
} 


$$
\sum_{Z} P L_{Z} X D_{Z}+\sum_{Z} \sum_{z j} P W_{z, z j} X M_{z, z j}=\sum_{z}\left(-C A B_{Z}\right)
$$

Substitute (18) into the last equation (equation (18) is no longer present among the model equations, but implied: equations (13), (15) and (3) together imply (4), and equations (4) and (17) together imply (18)). The substitution yields

$$
\sum_{z} P L_{z} X D_{z}+\sum_{z} \sum_{z j} P W_{z, z j} X M_{z, z j}=0
$$

So the total value of excess demands is zero, which is Walras' Law. It follows from Walras' Law that, if all excess demands but one are zero, then the remaining one is zero also. Therefore, one of the market equilibrium constraints consisting of (17) and $D_{Z}^{D}=D_{Z}^{O}$ is redundant (Of course, in the model, this equilibrium constraint is represented by the fact that both sides of the equation are one and the same variable, $D_{z}$ ).

However, rather than discarding one of the market equilibrium constraints, it is possible to discard one of the budget constraints, while retaining all market equilibrium constraints. Indeed, if all excess demands are zero for some price vector, the left-hand side of equation (B.35) is zero. It follows that, for any region $z, z \in\{1, \cdots, N\}$,

$$
\left(P C_{z} Q_{Z}-P_{Z} X S_{Z}\right)=-\sum_{z j \neq Z}\left(P C_{z j} Q_{z j}-P_{z j} X S_{z j}\right)
$$

while (18) implies

$$
C A B_{Z}=-\sum_{z j \neq z} C A B_{z j}
$$

Consequently, if $C A B_{z j}=P_{z j} X S_{z j}-P C_{z j} Q_{z j}$ for all $z j \neq z$, then the last two equations guarantee that, for the remaining region $z$ also, $C A B_{Z}=P_{Z} X S_{Z}-P C_{Z} Q_{Z}$. This is the form which Walras' Law takes in our model. We arbitrarily pick some region zleon, zleon $\in\{1, \cdots, N\}$ (zleon is a mnemonic for Léon Walras), and remove equation (3) for that single region. Note that, with the removal of the equation relating to zleon, the variable $C A B_{\text {zleon }}$ no longer appears in the model. Its value may be computed using the suppressed equation.

It is common practice in CGE modeling to introduce an extra variable and an extra equation to verify Walras' Law. In the GAMS implementation described in Appendix $\mathrm{K}$, the extra variable is labeled $L E O N$ in honor of Léon Walras, and $L E O N=C A B_{\text {zleon }}-\left(P_{\text {zleon }} X S_{\text {zleon }}-P C_{\text {zleon }} Q_{\text {zleon }}\right)$. A nonzero $L E O N$ in the solution indicates that there is an error in the model. 


\section{Appendix C: NLP problem with dummy objective}

Some modelers sidestep the task of seeking out and eliminating redundant equations by changing the constrained nonlinear system (CNS) into a nonlinear programming (NLP) problem with a dummy objective variable set equal to a constant. This is risky, because the solver will find a solution even if the model misses a closure equation, which usually implies that the solution produced by the solver depends on the initial values of the variables, because when a closure equation is missing, the solution is generally not unique. This point is illustrated here with the help of a simple example.

Consider the following example

$$
\begin{gathered}
x+y=a \\
b x+b y=b a \\
y=c
\end{gathered}
$$

where $a, b$ and $c$ are constants. Clearly, equation (C.2) is redundant given equation (C.1). Equation (C.3) plays the role of a closure rule. This model is programmed and solved following different strategies to illustrate the riskiness of using NLP. The GAMS code is given at the end of the appendix.

Using the GAMS solver CONOPT, with the statement

Solve testmodel_1 using CNS;

where testmodel_1 consists of equations (C.1), (C.2) and (C.3), the program aborts, because the model is not square: the CONOPT solver does not detect redundancy, so it believes there is one equation too many. On the other hand, with the statement

Solve testmodel_1 using NLP minimizing y;

the model finds a solution which is identical to the solution produced with the correct model testmodel_2, consisting of equations (C.1) and (C.3), by the statement

Solve testmodel_2 using CNS;

Now, suppose the modeler does not eliminate redundant equation (C.2), fails to include closure rule (C.3), and introduces a dummy objective variable, $z$. The resulting model is testmodel_3, which consists of equations (C.1) and (C.2), together with (C.4):

$$
z=d
$$

where $d$ is any constant. We now perform 
Solve testmodel_3 using NLP minimizing z;

Obviously, the solution of testmodel_3 is not unique. The solution produced by the algorithm depends on the initial values of variables. If the SOLVE statement for testmodel_3 comes immediately after the solution of testmodel_2, then $x$ and $y$ will be implicitly initialized at their correct solution values and the solution will be the same. But if the initial values are modified, then the solution could be different and, in our example, wrong.

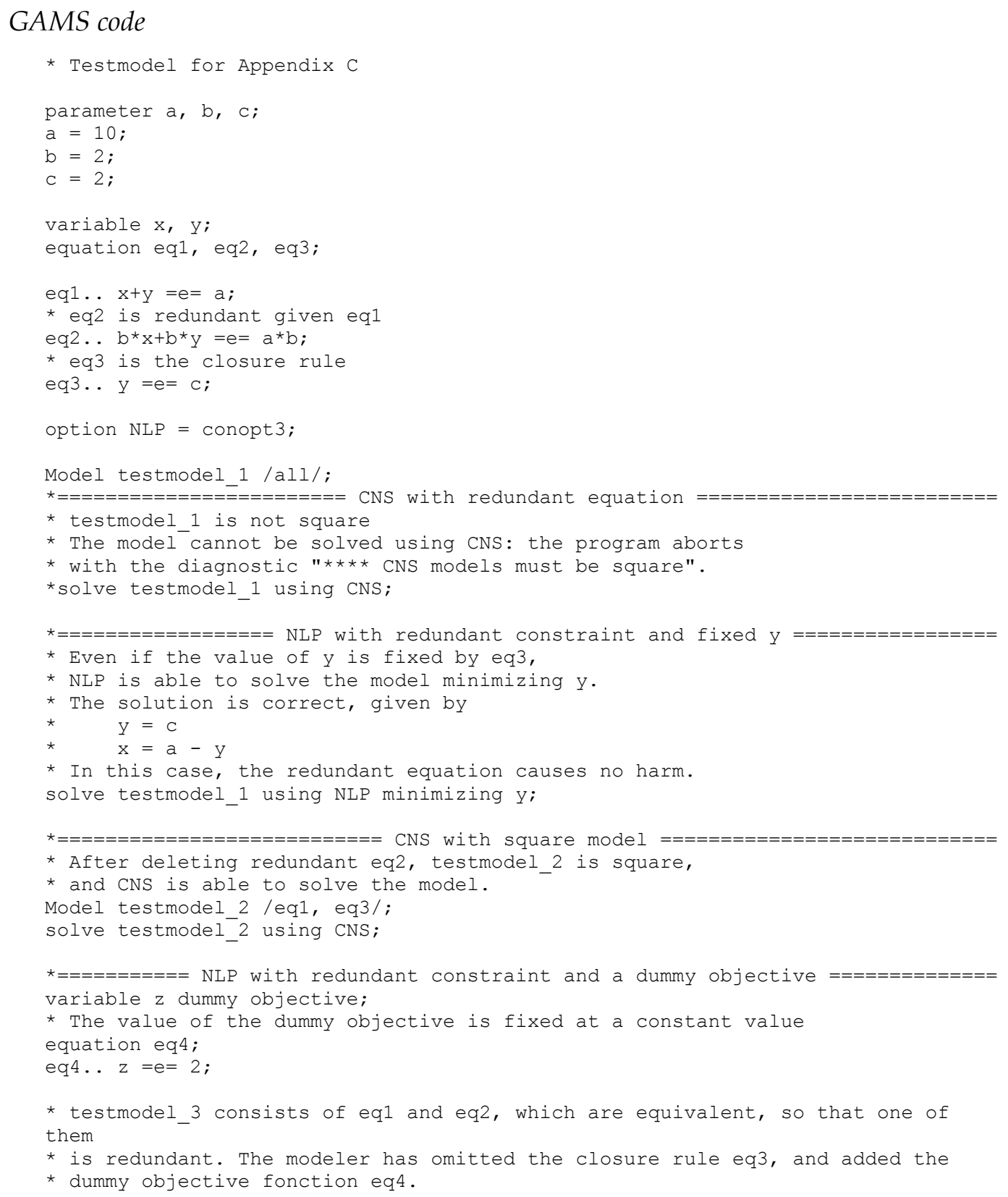


Journal of Global Economic Analysis, Volume 2 (2017), No. 1, pp. 215-324.

Model testmodel_3 /eq1, eq2, eq4/;

* The solution of the model is not unique, and it depends on initial values.

* For example, if $x$ and $y$ are not initialized, their value by default is

* their solution value from the preceding SOLVE:

parameter xSol, ySol;

$\mathrm{xSOl}=\mathrm{x} .1$

ySol $=y \cdot l$;

display xSol, ySol;

* Therefore, in this program, failing to initialize $x$ and y is equivalent to: $\mathrm{x} .1=8$;

$\mathrm{y} \cdot 1=2$;

* NLP finds the correct solution.

solve testmodel 3 using NLP minimizing $z$;

* But if the initial value of y were different, say

$\mathrm{x} .1=0$;

$\mathrm{y} \cdot 1=2$;

* then the solution violates missing eq3.

solve testmodel_3 using NLP minimizing z; 


\section{Appendix D: Equation (B.24)}

D.1 Cost minimizing problem

The regional agent allocates demand between domestic production and imports by minimizing consumption expenditures (equation (15)), subject to constraint (9).

D.2 Lagrangian and first-order conditions

Form the Lagrangian

$$
\begin{aligned}
\mathscr{L}_{Z} & =P L_{z} D_{Z}+P M T_{Z} I M T_{Z} \\
& -\mu_{z}\left\{A_{z}\left[\alpha_{Z} D_{z}^{-\rho_{z}}+\left(1-\alpha_{z}\right) I M T_{Z}^{-\rho_{z}}\right]^{-\frac{1}{\rho_{z}}}-Q_{Z}\right\}
\end{aligned}
$$

The first order conditions are:

$$
\begin{aligned}
& \frac{\partial \mathscr{L}_{z}}{\partial \mu_{z}}=-A_{z}\left[\alpha_{Z} D_{z}^{-\rho_{z}}+\left(1-\alpha_{z}\right) I M T_{Z}^{-\rho_{z}}\right]^{-\frac{1}{\rho_{z}}}+Q_{Z}=0 \\
& \frac{\partial \mathscr{L}_{z}}{\partial D_{Z}}=P L_{Z}-\mu_{z} \frac{\partial}{\partial D_{Z}} A_{z}\left[\alpha_{z} D_{z}^{-\rho_{z}}+\left(1-\alpha_{z}\right) I M T_{z}^{-\rho_{z}}\right]^{-\frac{1}{\rho_{z}}}=0 \\
& \frac{\partial \mathscr{L}_{Z}}{\partial I M T_{Z}}=P M T_{Z}- \\
& \mu_{z} \frac{\partial}{\partial I M T_{Z}} A_{z}\left[\alpha_{z} D_{z}^{-\rho_{z}}+\left(1-\alpha_{z}\right) I M T_{z}^{-\rho_{z}}\right]^{-\frac{1}{\rho_{Z}}}=0
\end{aligned}
$$

with 
Journal of Global Economic Analysis, Volume 2 (2017), No. 1, pp. 215-324.

$$
\begin{aligned}
& \frac{\partial}{\partial D_{z}} A_{z}\left[\alpha_{z} D_{z}^{-\rho_{z}}+\left(1-\alpha_{z}\right) I M T_{z}^{-\rho_{z}}\right]^{-\frac{1}{\rho_{Z}}}= \\
& -\frac{A_{Z}}{\rho_{Z}}\left[\alpha_{Z} D_{z}^{-\rho_{z}}+\left(1-\alpha_{z}\right) I M T_{Z}^{-\rho_{z}}\right]^{-\frac{1}{\rho_{Z}}-1} \\
& \frac{\partial}{\partial D_{z}}\left[\alpha_{z} D_{z}^{-\rho_{z}}+\left(1-\alpha_{z}\right) I M T_{z}^{-\rho_{z}}\right] \\
& \frac{\partial}{\partial D_{z}} A_{z}\left[\alpha_{z} D_{z}^{-\rho_{z}}+\left(1-\alpha_{z}\right) I M T_{z}^{-\rho_{z}}\right]^{-\frac{1}{\rho_{z}}}= \\
& A_{z}\left[\alpha_{z} D_{z}^{-\rho_{z}}+\left(1-\alpha_{z}\right) I M T_{z}^{-\rho_{z}}\right]^{-\frac{\rho_{Z}+1}{\rho_{z}}} \alpha_{z} D_{z}^{-\rho_{z}-1}
\end{aligned}
$$

and

$$
\begin{aligned}
& \frac{\partial}{\partial I M T_{Z}} A_{z}\left[\alpha_{z} D_{z}^{-\rho_{z}}+\left(1-\alpha_{z}\right) I M T_{z}^{-\rho_{z}}\right]^{-\frac{1}{\rho_{z}}}= \\
& -\frac{A_{z}}{\rho_{z}}\left[\alpha_{z} D_{z}^{-\rho_{z}}+\left(1-\alpha_{z}\right) I M T_{z}^{-\rho_{z}}\right]^{-\frac{1}{\rho_{z}}-1} \\
& \frac{\partial}{\partial I M T_{Z}}\left[\alpha_{z} D_{z}^{-\rho_{z}}+\left(1-\alpha_{z}\right) I M T_{z}^{-\rho_{z}}\right] \\
& \frac{\partial}{\partial I M T_{Z}} A_{z}\left[\alpha_{z} D_{z}^{-\rho_{z}}+\left(1-\alpha_{z}\right) I M T_{z}^{-\rho_{z}}\right]^{-\frac{1}{\rho_{z}}}= \\
& A_{Z}\left[\alpha_{Z} D_{Z}^{-\rho_{z}}+\left(1-\alpha_{Z}\right) I M T_{Z}^{-\rho_{z}}\right]^{-\frac{\rho_{Z}+1}{\rho_{Z}}}\left(1-\alpha_{Z}\right) I M T_{Z}^{-\rho_{Z}-1}
\end{aligned}
$$

Given (9), 


$$
\begin{gathered}
\frac{Q_{z}}{A_{z}}=\left[\alpha_{z} D_{z}^{-\rho_{z}}+\left(1-\alpha_{z}\right) I M T_{z}^{-\rho_{z}}\right]^{-\frac{1}{\rho_{z}}} \\
\left(\frac{Q_{z}}{A_{z}}\right)^{-\rho_{z}}=\left[\alpha_{z} D_{z}^{-\rho_{z}}+\left(1-\alpha_{z}\right) I M T_{z}^{-\rho_{z}}\right]
\end{gathered}
$$

Substitute the last equation into (D.6) to obtain

$$
\begin{gathered}
\frac{\partial}{\partial D_{z}} A_{z}\left[\alpha_{z} D_{z}{ }^{-\rho_{z}}+\left(1-\alpha_{z}\right) I M T_{z}{ }^{-\rho_{z}}\right]^{-\frac{1}{\rho_{z}}}= \\
A_{z}\left[\left(\frac{Q_{z}}{A_{z}}\right)^{-\rho_{z}}\right]^{-\frac{\rho_{z}+1}{\rho_{z}}} \alpha_{z} D_{z}^{-\rho_{z}-1} \\
\frac{\partial}{\partial D_{z}} A_{z}\left[\alpha_{z} D_{z}^{-\rho_{z}}+\left(1-\alpha_{z}\right) I M T_{z}^{-\rho_{z}}\right]^{-\frac{1}{\rho_{z}}}= \\
A_{z}\left(\frac{Q_{z}}{A_{z}}\right)^{\rho_{z}+1} \alpha_{z} D_{z}^{-\rho_{z}-1} \\
\frac{\partial}{\partial D_{z}} A_{z}\left[\alpha_{z} D_{z}^{-\rho_{z}}+\left(1-\alpha_{z}\right) I M T_{z}^{-\rho_{z}}\right]^{-\frac{1}{\rho_{z}}}=A_{z}^{-\rho_{z}} \alpha_{z}\left(\frac{Q_{z}}{D_{z}}\right)^{\rho_{z}+1}
\end{gathered}
$$

and similarly into (D.8), yielding

$$
\begin{gathered}
\frac{\partial}{\partial I M T_{Z}} A_{z}\left[\alpha_{Z} D_{z}^{-\rho_{z}}+\left(1-\alpha_{z}\right) I M T_{Z}^{-\rho_{z}}\right]^{-\frac{1}{\rho_{z}}}= \\
A_{Z}{ }^{-\rho_{z}}\left(1-\alpha_{z}\right)\left(\frac{Q_{z}}{I M T_{Z}}\right)^{\rho_{z}+1}
\end{gathered}
$$

First-order conditions (D.3) and (D.4) can now be written as 


$$
\begin{gathered}
\frac{\partial \mathscr{L}_{z}}{\partial D_{Z}}=P L_{z}-\mu_{z} A_{z}^{-\rho_{Z}} \alpha_{z}\left(\frac{Q_{Z}}{D_{z}}\right)^{\rho_{z}+1}=0 \\
\frac{\partial \mathscr{L}_{Z}}{\partial I M T_{Z}}=P M T_{Z}-\mu_{z} A_{z}^{-\rho_{z}}\left(1-\alpha_{z}\right)\left(\frac{Q_{Z}}{I M T_{Z}}\right)^{\rho_{z}+1}=0
\end{gathered}
$$

or

$$
\begin{gathered}
P L_{z}=\mu_{z} A_{z}^{-\rho_{z}} \alpha_{z}\left(\frac{Q_{z}}{D_{z}}\right)^{\rho_{z}+1} \\
P M T_{Z}=\mu_{z} A_{z}^{-\rho_{z}}\left(1-\alpha_{z}\right)\left(\frac{Q_{Z}}{I M T_{Z}}\right)^{\rho_{z}+1}
\end{gathered}
$$

D.3 Relative demand for domestic production and imports

Take the ratio of the final two equations of D.2,

$$
\begin{aligned}
& \frac{P L_{Z}}{P M T_{Z}}=\frac{\mu_{Z} A_{Z}{ }^{-\rho_{Z}} \alpha_{Z}\left(\frac{Q_{Z}}{D_{Z}}\right)^{\rho_{Z}+1}}{\mu_{Z} A_{Z}{ }^{-\rho_{Z}}\left(1-\alpha_{Z}\right)\left(\frac{Q_{Z}}{I M T_{Z}}\right)^{\rho_{z}+1}} \\
& \frac{P L_{z}}{P M T_{Z}}=\frac{\alpha_{Z} I M T_{Z}{ }^{\rho_{z}+1}}{\left(1-\alpha_{z}\right) D_{Z}{ }^{\rho_{z}+1}} \\
& \left(\frac{I M T_{Z}}{D_{Z}}\right)^{\rho_{z}+1}=\frac{\left(1-\alpha_{z}\right) P L_{Z}}{\alpha_{z} P M T_{Z}} \\
& \frac{I M T_{Z}}{D_{Z}}=\left(\frac{\left(1-\alpha_{z}\right) P L_{Z}}{\alpha_{z} P M T_{Z}}\right)^{\frac{1}{\rho_{Z}+1}}
\end{aligned}
$$

where $\rho_{z}=\frac{1-\sigma_{z}}{\sigma_{z}}$ implies $\rho_{z}+1=\frac{1}{\sigma_{z}}$, and 


$$
\begin{gathered}
\frac{I M T_{Z}}{D_{Z}}=\left(\frac{\left(1-\alpha_{Z}\right) P L_{z}}{\alpha_{z} P M T_{Z}}\right)^{\sigma_{z}} \\
I M T_{Z}=\left(\frac{\left(1-\alpha_{z}\right) P L_{z}}{\alpha_{z} P M T_{Z}}\right)^{\sigma_{z}} D_{Z}
\end{gathered}
$$

D.4 Demand for components in terms of composite demand and prices

Substitute the final equation of D.3 into (9)

$$
\begin{gathered}
Q_{z}=A_{z}\left\{\alpha_{z} D_{z}^{-\rho_{z}}+\left(1-\alpha_{z}\right)\left[\left(\frac{\left(1-\alpha_{z}\right) P L_{z}}{\alpha_{z} P M T_{z}}\right)^{\sigma_{z}} D_{z}\right]^{-\rho_{z}}\right\}^{-\frac{1}{\rho_{z}}} \\
Q_{Z}=A_{z} D_{z}\left\{\alpha_{z}+\left(1-\alpha_{z}\right)\left[\left(\frac{\left(1-\alpha_{z}\right) P L_{z}}{\alpha_{z} P M T_{z}}\right)^{\sigma_{z}}\right]^{-\rho_{z}}\right\}^{-\frac{1}{\rho_{z}}}
\end{gathered}
$$

where $\rho_{z}=\frac{1-\sigma_{z}}{\sigma_{z}}$ implies $\sigma_{z}=\frac{1}{\rho_{z}+1}$, and

$$
\begin{aligned}
& Q_{z}=A_{z} D_{z}\left\{\alpha_{z}+\left(1-\alpha_{z}\right)\left(\frac{\left(1-\alpha_{z}\right) P L_{z}}{\alpha_{z} P M T_{z}}\right)^{-\frac{\rho_{Z}}{\rho_{z}+1}}\right\}^{-\frac{1}{\rho_{z}}} \\
& \frac{D_{Z}}{Q_{Z}}=\frac{1}{A_{z}}\left\{\alpha_{Z}+\left(1-\alpha_{z}\right)\left(\frac{\left(1-\alpha_{z}\right) P L_{z}}{\alpha_{z} P M T_{z}}\right)^{-\frac{\rho_{z}}{\rho_{z}+1}}\right\}^{\frac{1}{\rho_{z}}}
\end{aligned}
$$




$$
\frac{D_{Z}}{Q_{Z}}=\frac{1}{A_{z}}\left\{\left(\frac{\alpha_{Z}}{P L_{z}}\right)^{\frac{\rho_{Z}}{\rho_{Z}+1}}\left[\begin{array}{c}
\alpha_{Z}\left(\frac{\alpha_{Z}}{P L_{Z}}\right)^{-\frac{\rho_{Z}}{\rho_{Z}+1}} \\
+\left(1-\alpha_{Z}\right)\left(\frac{\left(1-\alpha_{Z}\right)}{P M T_{Z}}\right)^{-\frac{\rho_{Z}}{\rho_{z}+1}}
\end{array}\right]\right\}^{\frac{1}{\rho_{Z}}}
$$

where $\rho_{z}=\frac{1-\sigma_{z}}{\sigma_{z}}$ implies $\rho_{z}+1=\frac{1}{\sigma_{z}}$, and $\frac{\rho_{z}}{\rho_{z}+1}=1-\sigma_{z}$. It follows that

$$
\begin{aligned}
& \frac{D_{z}}{Q_{z}}=\frac{1}{A_{z}}\left(\frac{\alpha_{z}}{P L_{z}}\right)^{\sigma_{z}}\left[\begin{array}{l}
\alpha_{z}\left(\frac{\alpha_{z}}{P L_{z}}\right)^{\sigma_{z}-1} \\
+\left(1-\alpha_{z}\right)\left(\frac{\left(1-\alpha_{z}\right)}{P M T_{z}}\right)^{\sigma_{z}-1}
\end{array}\right]^{-\frac{\sigma_{z}}{\sigma_{z}-1}} \\
& \frac{D_{z}}{Q_{z}}=\frac{1}{A_{z}}\left(\frac{\alpha_{z}}{P L_{z}}\right)^{\sigma_{z}}\left[P L_{z}\left(\frac{\alpha_{z}}{P L_{z}}\right)^{\sigma_{z}}+P M T_{z}\left(\frac{\left(1-\alpha_{z}\right)}{P M T_{z}}\right)^{\sigma_{z}}\right]^{-\frac{\sigma_{z}}{\sigma_{z}-1}}
\end{aligned}
$$

where the left-hand side ratio depends only on component prices. As a matter of fact, multiplying both sides of (D.33) by $Q_{z}$ transforms it into a demand equation of $D_{Z}$. A similar development would lead to a demand equation for $I M T_{Z}$. 


$$
\begin{aligned}
& \frac{I M T_{z}}{Q_{z}}= \\
& \frac{1}{A_{z}}\left(\frac{\left(1-\alpha_{z}\right)}{P M T_{z}}\right)^{\sigma_{z}}\left[P L_{z}\left(\frac{\alpha_{z}}{P L_{z}}\right)^{\sigma_{z}}+P M T_{z}\left(\frac{\left(1-\alpha_{z}\right)}{P M T_{Z}}\right)^{\sigma_{z}}\right]^{-\frac{\sigma_{z}}{\sigma_{z}-1}}
\end{aligned}
$$

\section{D.5 Unit cost of composite demand}

It is clear from (D.23) that the relative demand for domestic production and imports is independent of the scale of demand, which is consistent with the firstdegree homogeneity of aggregator function (9). Consequently, the unit cost of the composite demand can be obtained from (15) by substituting the optimal ratio determined in (D.23), and dividing through by $Q_{z}$.

$$
\begin{gathered}
P C_{z} Q_{z}=\left(P L_{z}+P M T_{z} \frac{I M T_{z}}{D_{z}}\right) D_{z} \\
P C_{z} Q_{z}=\left[P L_{z}+P M T_{Z}\left(\frac{\left(1-\alpha_{z}\right) P L_{z}}{\alpha_{z} P M T_{z}}\right)^{\sigma_{z}}\right] D_{z} \\
P C_{z}=\left[P L_{z}+P M T_{z}\left(\frac{\left(1-\alpha_{z}\right) P L_{z}}{\alpha_{z} P M T_{z}}\right)^{\sigma_{z}}\right] \frac{D_{z}}{Q_{z}} \\
P C_{z}=\left(\frac{P L_{z}}{\alpha_{z}}\right)^{\sigma_{z}}\left[P L_{z}\left(\frac{\alpha_{z}}{P L_{z}}\right)^{\sigma_{z}}+P M T_{z}\left(\frac{\left(1-\alpha_{z}\right)}{P M T_{z}}\right)^{\sigma_{z}}\right] \frac{D_{z}}{Q_{z}}
\end{gathered}
$$

To express $P C_{z}$ in terms of the component prices only, we must substitute for $D_{z} / Q_{z}$ in this last equation using (D.33): 


$$
\begin{aligned}
& P C_{Z}=\left(\frac{P L_{Z}}{\alpha_{z}}\right)^{\sigma_{Z}}\left[P L_{Z}\left(\frac{\alpha_{z}}{P L_{Z}}\right)^{\sigma_{z}}+P M T_{Z}\left(\frac{\left(1-\alpha_{z}\right)}{P M T_{Z}}\right)^{\sigma_{z}}\right] \\
& \frac{1}{A_{z}}\left(\frac{\alpha_{z}}{P L_{z}}\right)^{\sigma_{z}}\left[P L_{z}\left(\frac{\alpha_{z}}{P L_{z}}\right)^{\sigma_{z}}+P M T_{Z}\left(\frac{\left(1-\alpha_{z}\right)}{P M T_{Z}}\right)^{\sigma_{z}}\right]^{\frac{\sigma_{z}}{\sigma_{z}-1}} \\
& P C_{z}=\frac{1}{A_{z}}\left[P L_{z}\left(\frac{\alpha_{z}}{P L_{z}}\right)^{\sigma_{z}}+P M T_{z}\left(\frac{\left(1-\alpha_{z}\right)}{P M T_{z}}\right)^{\sigma_{z}}\right]^{1+\frac{\sigma_{Z}}{\sigma_{z}-1}} \\
& P C_{Z}=\frac{1}{A_{z}}\left[P L_{z}\left(\frac{\alpha_{Z}}{P L_{Z}}\right)^{\sigma_{z}}+P M T_{Z}\left(\frac{\left(1-\alpha_{z}\right)}{P M T_{Z}}\right)^{\sigma_{z}}\right]^{-\frac{1}{\sigma_{Z}-1}}
\end{aligned}
$$

\section{D.6 Demand for domestic production and imports reformulated}

Given the last equation in D.5, we have

$$
A_{z} P C_{z}=\left[P L_{z}\left(\frac{\alpha_{z}}{P L_{z}}\right)^{\sigma_{z}}+P M T_{z}\left(\frac{\left(1-\alpha_{z}\right)}{P M T_{z}}\right)^{\sigma_{z}}\right]^{-\frac{1}{\sigma_{z}-1}}
$$

Using the preceding equation, we can write (D.33) as

$$
\begin{gathered}
\frac{D_{Z}}{Q_{Z}}=\frac{1}{A_{Z}}\left(\frac{\alpha_{Z}}{P L_{Z}}\right)^{\sigma_{z}}\left(A_{Z} P C_{Z}\right)^{\sigma_{Z}} \\
\frac{D_{Z}}{Q_{Z}}=\left(A_{Z}\right)^{\sigma_{z}-1}\left(\frac{\alpha_{Z} P C_{Z}}{P L_{z}}\right)^{\sigma_{z}}
\end{gathered}
$$




$$
D_{z}=\left(A_{z}\right)^{\sigma_{z}-1}\left(\frac{\alpha_{z} P C_{z}}{P L_{z}}\right)^{\sigma_{z}} Q_{z}
$$

which is an alternate form of the demand equation for domestic production. A similar development leads to the import demand equation:

$$
I M T_{Z}=\left(A_{Z}\right)^{\sigma_{Z}-1}\left(\frac{\left(1-\alpha_{z}\right) P C_{Z}}{P M T_{Z}}\right)^{\sigma_{Z}} Q_{Z}
$$

Equation (B.24) defines $D_{Z}^{D}$ from (D.43). 


\section{Appendix E: Equation (B.25)}

E.1 Sales revenue maximizing problem

The regional agent allocates production between the domestic market and exports by maximizing the value of production (equation (13)), subject to constraint (5).

E.2 Lagrangian and first-order conditions

Form the Lagrangian

$$
\begin{aligned}
\mathscr{L}_{z} & =P L_{z} D_{z}+P X T_{z} E X T \\
& -\lambda_{z}\left\{B_{z}\left[\beta_{z} D_{z}^{\kappa_{z}}+\left(1-\beta_{z}\right) E X T_{z}{ }^{\kappa_{z}}\right]^{\frac{1}{\kappa_{z}}}-X S_{z}\right\}
\end{aligned}
$$

The first order conditions are:

$$
\begin{aligned}
& \frac{\partial \mathscr{L}_{Z}}{\partial \lambda_{z}}=-B_{Z}\left[\beta_{Z} D_{Z}{ }^{\kappa_{z}}+\left(1-\beta_{Z}\right) E X T_{Z}{ }^{\kappa_{z}}\right]^{\frac{1}{\kappa_{z}}}+X S_{Z}=0 \\
& \frac{\partial \mathscr{L}_{z}}{\partial D_{z}}=P L_{z}-\lambda_{z} \frac{\partial}{\partial D_{z}} B_{z}\left[\beta_{z} D_{z} \kappa_{z}+\left(1-\beta_{z}\right) E X T_{z}{ }^{\kappa_{z}}\right]^{\frac{1}{\kappa_{z}}}=0 \\
& \frac{\partial \mathscr{L}_{Z}}{\partial E X T_{Z}}=P X T_{Z}-\lambda_{z} \frac{\partial}{\partial E X T_{Z}} B_{Z}\left[\beta_{Z} D_{Z}{ }^{\kappa_{z}}+\left(1-\beta_{Z}\right) E X T_{Z}{ }^{\kappa_{z}}\right]^{\frac{1}{\kappa_{Z}}}=0
\end{aligned}
$$

with 
Journal of Global Economic Analysis, Volume 2 (2017), No. 1, pp. 215-324.

$$
\begin{aligned}
& \frac{\partial}{\partial D_{Z}} B_{z}\left[\beta_{z} D_{z} \kappa_{z}+\left(1-\beta_{z}\right) E X T_{Z} \kappa_{z}^{\frac{1}{\kappa_{z}}}=\right. \\
& \frac{B_{Z}}{\kappa_{Z}}\left[\beta_{z} D_{z} \kappa_{z}+\left(1-\beta_{z}\right) E X T_{z}{ }^{\kappa_{z}}\right]^{\frac{1}{\kappa_{z}}-1} \\
& \frac{\partial}{\partial D_{z}}\left[\beta_{z} D_{z}{ }^{\kappa_{z}}+\left(1-\beta_{z}\right) E X T_{z}{ }^{\kappa_{z}}\right] \\
& \frac{\partial}{\partial D_{z}} B_{z}\left[\beta_{z} D_{z}{ }^{\kappa_{z}}+\left(1-\beta_{z}\right) E X T_{z}{ }^{\kappa_{z}}\right]^{\frac{1}{\kappa_{z}}}= \\
& B_{Z}\left[\beta_{Z} D_{Z}{ }^{\kappa_{z}}+\left(1-\beta_{Z}\right) E X T_{Z}{ }^{\kappa_{z}}\right]^{\frac{1}{\kappa_{z}}-1} \beta_{Z} D_{Z}{ }^{\kappa_{z}-1}
\end{aligned}
$$

and

$$
\begin{aligned}
& \frac{\partial}{\partial E X T_{Z}} B_{Z}\left[\beta_{Z} D_{Z}{ }^{\kappa_{z}}+\left(1-\beta_{Z}\right) E X T_{Z}{ }^{\kappa_{z}}\right]^{\frac{1}{\kappa_{Z}}}= \\
& \frac{B_{Z}}{\kappa_{Z}}\left[\beta_{Z} D_{Z}^{\kappa_{z}}+\left(1-\beta_{Z}\right) E X T_{Z}{ }^{\kappa_{z}}\right]^{\frac{1}{\kappa_{z}}-1} \\
& \frac{\partial}{\partial E X T_{Z}}\left[\beta_{Z} D_{z}^{\kappa_{z}}+\left(1-\beta_{z}\right) E X T_{Z}^{\kappa_{z}}\right] \\
& \frac{\partial}{\partial E X T_{Z}} B_{z}\left[\beta_{z} D_{z}^{\kappa_{z}}+\left(1-\beta_{z}\right) E X T_{Z}{ }^{\kappa_{z}}\right]^{\frac{1}{\kappa_{z}}}= \\
& B_{Z}\left[\beta_{Z} D_{Z}{ }^{\kappa_{z}}+\left(1-\beta_{Z}\right) E X T_{Z}{ }^{\kappa_{z}}\right]^{\frac{1}{\kappa_{Z}}-1}\left(1-\beta_{z}\right) E X T_{Z}{ }^{\kappa_{z}-1}
\end{aligned}
$$

Given (5), 


$$
\begin{gathered}
\frac{X S_{Z}}{B_{Z}}=\left[\beta_{Z} D_{Z}{ }^{\kappa_{Z}}+\left(1-\beta_{Z}\right) E X T_{Z}{ }^{\kappa_{Z}}\right]^{\frac{1}{\kappa_{Z}}} \\
\left(\frac{X S_{Z}}{B_{Z}}\right)^{\kappa_{Z}}=\left[\beta_{Z} D_{Z}{ }^{\kappa_{Z}}+\left(1-\beta_{Z}\right) E X T_{Z}{ }^{\kappa_{Z}}\right]
\end{gathered}
$$

Substitute that into (E.6) to obtain

$$
\begin{aligned}
& \frac{\partial}{\partial D_{z}} B_{Z}\left[\beta_{Z} D_{z}{ }^{\kappa_{z}}+\left(1-\beta_{Z}\right) E X T_{Z}{ }^{\kappa_{z}}\right]^{\frac{1}{\kappa_{Z}}}= \\
& B_{Z}\left[\left(\frac{X S_{Z}}{B_{Z}}\right)^{\kappa_{Z}}\right]^{\frac{1}{\kappa_{z}}-1} \beta_{Z} D_{z}{ }^{\kappa_{z}-1} \\
& \frac{\partial}{\partial D_{z}} B_{z}\left[\beta_{Z} D_{Z}{ }^{\kappa_{z}}+\left(1-\beta_{Z}\right) E X T_{Z}{ }^{\kappa_{z}}\right]^{\frac{1}{\kappa_{Z}}}=B_{Z}{ }^{\kappa_{z}} X S_{z}{ }^{1-\kappa_{z}} \beta_{Z} D_{Z}{ }^{\kappa_{z}-1}
\end{aligned}
$$

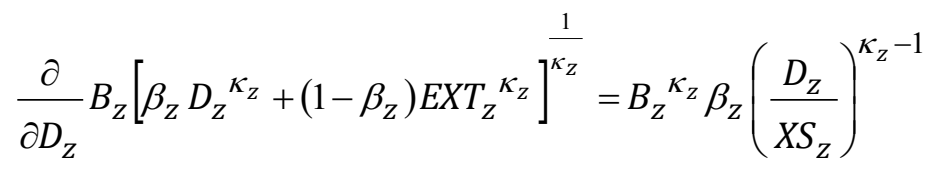

and similarly into (E.8), which yields

$$
\begin{gathered}
\frac{\partial}{\partial E X T_{Z}} B_{Z}\left[\beta_{Z} D_{Z}{ }^{\kappa_{z}}+\left(1-\beta_{Z}\right) E X T_{Z}{ }^{\kappa_{z}}\right]^{\frac{1}{\kappa_{Z}}}= \\
B_{Z}{ }^{\kappa_{z}}\left(1-\beta_{Z}\right)\left(\frac{E X T_{Z}}{X S_{Z}}\right)^{\kappa_{z}-1}
\end{gathered}
$$

First-order conditions (E.3) and (E.4) can now be written as

$$
\frac{\partial \mathscr{L}_{z}}{\partial D_{Z}}=P L_{Z}-\lambda_{Z} B_{Z}{ }^{\kappa_{z}} \beta_{Z}\left(\frac{D_{Z}}{X S_{Z}}\right)^{\kappa_{Z}-1}=0
$$




$$
\frac{\partial \mathscr{L}_{Z}}{\partial E X T_{Z}}=P X T_{Z}-\lambda_{Z} B_{Z}{ }^{\kappa_{z}}\left(1-\beta_{Z}\right)\left(\frac{E X T_{Z}}{X S_{Z}}\right)^{\kappa_{z}-1}=0
$$

or

$$
\begin{gathered}
P L_{Z}=\lambda_{Z} B_{Z}{ }^{\kappa_{z}} \beta_{Z}\left(\frac{D_{Z}}{X S_{Z}}\right)^{\kappa_{Z}-1} \\
P X T_{Z}=\lambda_{Z} B_{Z}{ }^{\kappa_{z}}\left(1-\beta_{Z}\right)\left(\frac{E X T_{Z}}{X S_{Z}}\right)^{K_{Z}-1}
\end{gathered}
$$

E.3 Relative supply of components

Take the ratio of the two last equations in E.2:

$$
\begin{aligned}
& \frac{P L_{Z}}{P X T_{Z}}=\frac{\lambda_{Z} B_{Z}{ }^{{ }^{K}} \beta_{Z}\left(\frac{D_{Z}}{X S_{Z}}\right)^{\kappa_{Z}-1}}{\lambda_{Z} B_{Z}{ }^{{ }^{K}}{ }\left(1-\beta_{Z}\right)\left(\frac{E X T_{Z}}{X S_{Z}}\right)^{K_{Z}-1}} \\
& \frac{P L_{Z}}{P X T_{Z}}=\frac{\beta_{Z}\left(D_{Z}\right)^{K_{Z}-1}}{\left(1-\beta_{Z}\right)\left(E X T_{Z}\right)^{K_{Z}-1}} \\
& \frac{\left(D_{z}\right)^{K_{z}-1}}{\left(E X T_{z}\right)^{K_{z}-1}}=\frac{\left(1-\beta_{z}\right) P L_{z}}{\beta_{z} P X T_{Z}} \\
& \frac{D_{Z}}{E X T_{Z}}=\left(\frac{\left(1-\beta_{Z}\right) P L_{Z}}{\beta_{Z} P X T_{Z}}\right)^{\frac{1}{\kappa_{Z}-1}}
\end{aligned}
$$

where $\kappa_{z}=\frac{\tau_{z}+1}{\tau_{z}}$ implies $\kappa_{z}-1=\frac{1}{\tau_{z}}$ and

$$
\frac{D_{Z}}{E X T_{Z}}=\left(\frac{\left(1-\beta_{Z}\right) P L_{Z}}{\beta_{Z} P X T_{Z}}\right)^{\tau_{Z}}
$$




$$
D_{z}=\left(\frac{\left(1-\beta_{Z}\right) P L_{z}}{\beta_{Z} P X T_{Z}}\right)^{\tau_{z}} E X T_{Z}
$$

E.4 Supply of components in terms of aggregate production and prices

Substitute the last equation of E.3 into (5)

$$
\begin{gathered}
X S_{Z}=B_{Z}\left\{\beta_{Z}\left[\left(\frac{\left(1-\beta_{Z}\right) P L_{Z}}{\beta_{Z} P X T_{Z}}\right)^{\tau_{Z}} E X T_{Z}\right]^{\kappa_{Z}}+\left(1-\beta_{Z}\right) E X T_{Z}{ }^{\kappa_{z}}\right\}^{\frac{1}{\kappa_{Z}}} \\
X S_{Z}=B_{Z} \operatorname{EXT}_{Z}\left\{\beta_{Z}\left[\left(\frac{\left(1-\beta_{Z}\right) P L_{Z}}{\beta_{Z} P X T_{Z}}\right)^{\tau_{Z}}\right]^{\frac{1}{\kappa_{z}}}+\left(1-\beta_{Z}\right)\right\}^{\kappa_{z}}
\end{gathered}
$$

where $\kappa_{z}=\frac{\tau_{z}+1}{\tau_{z}}$ implies $\tau_{z}=\frac{1}{\kappa_{z}-1}$ and

$$
\begin{aligned}
& X S_{Z}=B_{Z} E X T_{Z}\left\{\beta_{Z}\left[\left(\frac{\left(1-\beta_{Z}\right) P L_{Z}}{\beta_{Z} P X T_{Z}}\right)^{\frac{1}{\kappa_{Z}-1}}\right]^{\kappa_{Z}}+\left(1-\beta_{Z}\right)\right\}^{\frac{1}{\kappa_{Z}}} \\
& X S_{Z}=B_{Z} E X T_{Z}\left\{\beta_{Z}\left(\frac{\left(1-\beta_{Z}\right) P L_{Z}}{\beta_{Z} P X T_{Z}}\right)^{\frac{\kappa_{Z}}{\kappa_{z}-1}}+\left(1-\beta_{z}\right)\right\}^{\frac{1}{\kappa_{z}}}
\end{aligned}
$$


Journal of Global Economic Analysis, Volume 2 (2017), No. 1, pp. 215-324.

$$
\begin{aligned}
& \frac{E X T_{Z}}{X S_{Z}}=\frac{1}{B_{Z}}\left\{\beta_{Z}\left(\frac{\left(1-\beta_{Z}\right) P L_{Z}}{\beta_{Z} P X T_{Z}}\right)^{\frac{\kappa_{Z}}{\kappa_{Z}-1}}+\left(1-\beta_{Z}\right)\right\}^{-\frac{1}{\kappa_{Z}}}
\end{aligned}
$$

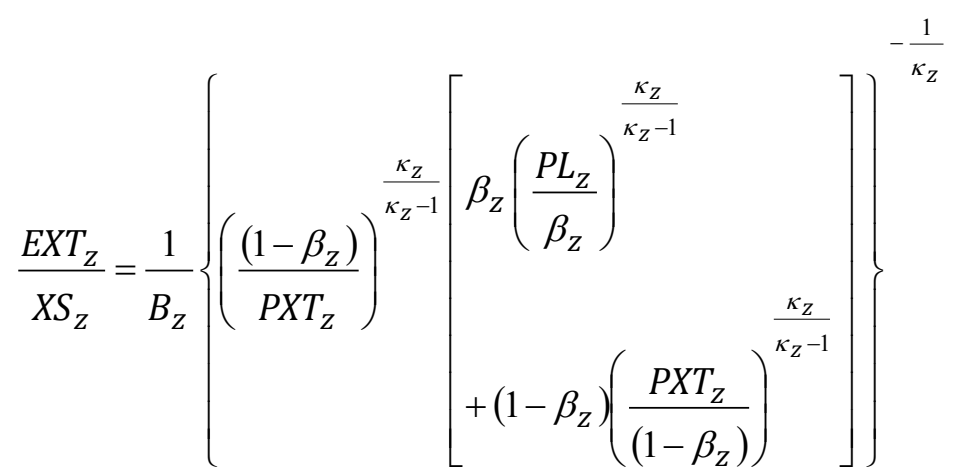

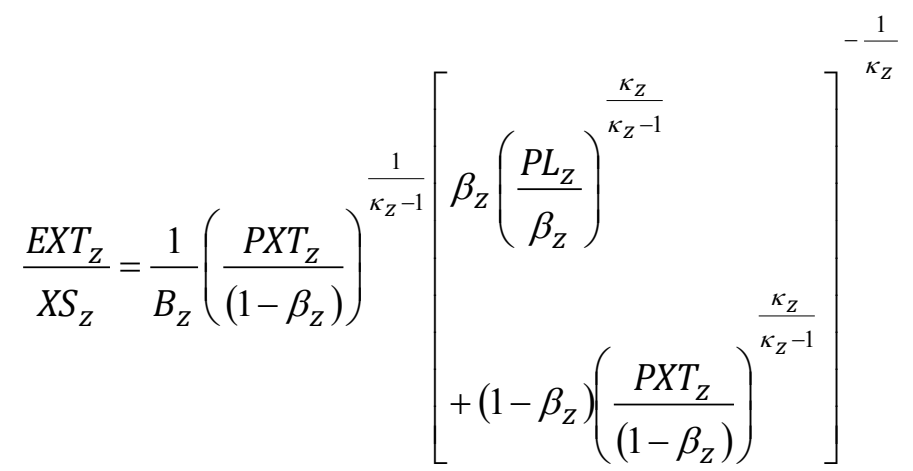

where $\kappa_{z}=\frac{\tau_{z}+1}{\tau_{z}}$ implies $\kappa_{z}-1=\frac{1}{\tau_{z}}$ and $\frac{\kappa_{z}}{\kappa_{z}-1}=\tau_{z}+1$. It follows that

$$
\frac{E X T_{Z}}{X S_{Z}}=\frac{1}{B_{Z}}\left(\frac{P X T_{Z}}{\left(1-\beta_{Z}\right)}\right)^{\tau_{Z}}\left[\beta_{Z}\left(\frac{P L_{Z}}{\beta_{Z}}\right)^{\tau_{Z}+1}+\left(1-\beta_{Z}\right)\left(\frac{P X T_{Z}}{\left(1-\beta_{Z}\right)}\right)^{\tau_{Z}+1}\right]^{-\frac{\tau_{Z}}{\tau_{Z}+1}}
$$




$$
\frac{E X T_{Z}}{X S_{Z}}=\frac{1}{B_{Z}}\left(\frac{P X T_{Z}}{\left(1-\beta_{z}\right)}\right)^{\tau_{Z}}\left[P L_{z}\left(\frac{P L_{z}}{\beta_{z}}\right)^{\tau_{Z}}+P X T_{Z}\left(\frac{P X T_{Z}}{\left(1-\beta_{z}\right)}\right)^{\tau_{z}}\right]^{-\frac{\tau_{Z}}{\tau_{z}+1}}
$$

where the left-hand side ratio depends only on component prices. As a matter of fact, multiplying both sides of (E.33) by $X S_{z}$ transforms it into a supply equation of $E X T_{Z}$. A similar development would lead to a supply equation for $D_{z}$.

$$
\frac{D_{Z}}{X S_{Z}}=\frac{1}{B_{Z}}\left(\frac{P L_{Z}}{\beta_{Z}}\right)^{\tau_{Z}}\left[P L_{Z}\left(\frac{P L_{Z}}{\beta_{Z}}\right)^{\tau_{Z}}+P X T_{Z}\left(\frac{P X T_{Z}}{\left(1-\beta_{Z}\right)}\right)^{\tau_{Z}}\right]^{-\frac{\tau_{Z}}{\tau_{Z}+1}}
$$

\section{E.5 Unit value of aggregate output}

It is clear from (E.23) that the relative supply on the domestic and export markets is independent of the scale of output, consistent with the first-degree homogeneity of aggregator function (5). Consequently, the unit value of the output aggregate can be obtained from (13) by substituting the optimal ratio determined in (E.23), and dividing through by $X S_{Z}$.

$$
\begin{gathered}
P_{Z} X S_{Z}=\left(P L_{z} \frac{D_{Z}}{E X T_{Z}}+P X T_{Z}\right) E X T_{Z} \\
P_{Z} X S_{Z}=\left[P L_{Z}\left(\frac{\left(1-\beta_{Z}\right) P L_{Z}}{\beta_{Z} P X T_{Z}}\right)^{\tau_{Z}}+P X T_{Z}\right] E X T_{Z} \\
P_{Z}=\left[P L_{Z}\left(\frac{\left(1-\beta_{Z}\right) P L_{Z}}{\beta_{Z} P X T_{Z}}\right)^{\tau_{Z}}+P X T_{Z}\right] \frac{E X T_{Z}}{X S_{Z}} \\
P_{Z}=\left(\frac{\left(1-\beta_{Z}\right)}{P X T_{Z}}\right)^{\tau_{Z}}\left[P L_{Z}\left(\frac{P L_{Z}}{\beta_{Z}}\right)^{\tau_{Z}}+P X T_{Z}\left(\frac{P X T_{Z}}{\left(1-\beta_{Z}\right)}\right)^{\tau_{z}}\right] \frac{E X T_{Z}}{X S_{Z}}
\end{gathered}
$$

To express $P_{z}$ in terms of the component prices only, we must substitute for $E X T_{Z} / X S_{Z}$ in the preceding equation using (E.33): 


$$
\begin{gathered}
P_{Z}=\left(\frac{\left(1-\beta_{Z}\right)}{P X T_{Z}}\right)^{\tau_{Z}}\left[P L_{Z}\left(\frac{P L_{Z}}{\beta_{Z}}\right)^{\tau_{Z}}+P X T_{Z}\left(\frac{P X T_{Z}}{\left(1-\beta_{Z}\right)}\right)^{\tau_{Z}}\right] \\
\frac{1}{B_{Z}}\left(\frac{P X T_{Z}}{\left(1-\beta_{Z}\right)}\right)^{\tau_{Z}}\left[P L_{Z}\left(\frac{P L_{Z}}{\beta_{Z}}\right)^{\tau_{Z}}+P X T_{Z}\left(\frac{P X T_{Z}}{\left(1-\beta_{Z}\right)}\right)^{\tau_{Z}}\right]^{-\frac{\tau_{Z}}{\tau_{Z}+1}} \\
P_{Z}=\frac{1}{B_{Z}}\left[P L_{Z}\left(\frac{P L_{Z}}{\beta_{Z}}\right)^{\tau_{Z}}+P X T_{Z}\left(\frac{P X T_{Z}}{\left(1-\beta_{Z}\right)}\right)^{\tau_{Z}}\right] \\
\left.\left.P_{Z}=\frac{1}{B_{Z}}\left[P L_{Z}\left(\frac{P L_{Z}}{\beta_{Z}}\right)^{\tau_{Z}}+P X T_{Z}\left(\frac{P X T_{Z}}{\left(1-\beta_{Z}\right)}\right)^{\tau_{Z}}\right]^{\frac{P L_{Z}}{\beta_{Z}}}\right)^{\tau_{Z}}+P X T_{Z}\left(\frac{P X T_{Z}}{\left(1-\beta_{Z}\right)}\right)^{\tau_{Z}}\right]^{-\frac{\tau_{Z}}{\tau_{Z}+1}}
\end{gathered}
$$

E.6 Supply of components reformulated

Given the last equation in E.5, we have

$$
B_{Z} P_{Z}=\left[P L_{Z}\left(\frac{P L_{Z}}{\beta_{Z}}\right)^{\tau_{Z}}+P X T_{Z}\left(\frac{P X T_{Z}}{\left(1-\beta_{Z}\right)}\right)^{\tau_{Z}}\right]^{\frac{1}{\tau_{z}+1}}
$$

Using this, we can write (E.33) as

$$
\begin{aligned}
& \frac{E X T_{Z}}{X S_{Z}}=\frac{1}{B_{Z}}\left(\frac{P X T_{Z}}{\left(1-\beta_{Z}\right)}\right)^{\tau_{Z}}\left(B_{Z} P_{Z}\right)^{-\tau_{Z}} \\
& \frac{E X T_{Z}}{X S_{Z}}=\left(\frac{1}{B_{Z}}\right)^{\tau_{Z}+1}\left(\frac{P X T_{Z}}{\left(1-\beta_{Z}\right) P_{Z}}\right)^{\tau_{Z}}
\end{aligned}
$$




$$
E X T_{Z}=\left(\frac{1}{B_{Z}}\right)^{\tau_{Z}+1}\left(\frac{P X T_{Z}}{\left(1-\beta_{Z}\right) P_{Z}}\right)^{\tau_{Z}} X S_{Z}
$$

which is an alternate form of the export supply equation. A similar development leads to the domestic supply equation:

$$
D_{Z}=\left(\frac{1}{B_{Z}}\right)^{\tau_{Z}+1}\left(\frac{P L_{Z}}{\beta_{Z} P_{Z}}\right)^{\tau_{Z}} X S_{Z}
$$

Equation (B.25) defines $D_{Z}^{O}$ from (E.46). 


\section{Appendix F: Equation (B.26)}

F.1 Cost minimizing problem

The regional agent allocates imports between origins by minimizing the aggregate cost of imports (equation (16)) subject to constraint (11) and to $I M T_{Z}=I M T_{Z}^{*}$, where $I M T_{Z}^{*}$ is the solution to the demand allocation problem as given by (D.44).

F.2 Lagrangian and first-order conditions

Write the Lagrangian

$$
\mathscr{L}_{Z}=\sum_{z j} P W_{z j, z} I M_{z j, z}-\mu_{z}^{M}\left\{A_{Z}^{M}\left[\sum_{z j} \alpha_{z j, z}^{M} I M_{z j, z}^{-\rho_{Z}^{M}}\right]^{\frac{-1}{\rho_{Z}^{M}}}-I M T_{z}^{*}\right\}
$$

The first order conditions are:

$$
\begin{gathered}
\frac{\partial \mathscr{L}_{z}}{\partial \mu_{Z}^{X}}=-A_{z}^{M}\left[\sum_{z j} \alpha_{z j, z}^{M} I M_{z j, z}^{-\rho_{z}^{M}}\right]^{\frac{-1}{\rho_{Z}^{M}}}+I M T_{z}^{*}=0 \\
\frac{\partial \mathscr{L}_{z}}{\partial I M_{z j, z}}=P W_{z j, z}-\mu_{z}^{M} \frac{\partial}{\partial I M_{z j, z}} A_{z}^{M}\left[\sum_{z j j} \alpha_{z j j, z}^{M} I M_{z j j, z}^{-\rho_{Z}^{M}}\right]^{\frac{-1}{\rho_{Z}^{M}}}=0
\end{gathered}
$$

where

$$
\begin{aligned}
& \frac{\partial}{\partial I M_{z j, z}} A_{z}^{M}\left[\sum_{z j j} \alpha_{z j j, z}^{M} I M_{z j j, z}^{-\rho_{Z}^{M}}\right]^{\frac{-1}{\rho_{Z}^{M}}}= \\
& -A_{Z}^{M} \frac{1}{\rho_{z}^{M}}\left[\sum_{z j j} \alpha_{z j j, z}^{M} I M_{z j j, z}^{-\rho_{Z}^{M}}\right]^{\frac{-1}{\rho_{Z}^{M}-1}} \frac{\partial}{\partial I M_{z j, z}}\left[\sum_{z j j} \alpha_{z j j, z}^{M} I M_{z j j, z}^{-\rho_{Z}^{M}}\right]
\end{aligned}
$$


Journal of Global Economic Analysis, Volume 2 (2017), No. 1, pp. 215-324.

$$
\begin{gathered}
\frac{\partial}{\partial I M_{z j, z}} A_{Z}^{M}\left[\sum_{z j j} \alpha_{z j j, z}^{M} I M_{z j j, z}^{-\rho_{z}^{M}}\right]^{\frac{-1}{\rho_{Z}^{M}}}= \\
-A_{z}^{M} \frac{1}{\rho_{z}^{M}}\left[\sum_{z j j} \alpha_{z j j, z}^{M} I M_{z j j, z}^{-\rho_{z}^{M}}\right]^{\frac{-1}{\rho_{Z}^{M}-1}} \alpha_{z j, z}^{M}\left(-\rho_{z}^{M}\right) I M_{z j, z}^{-\rho_{Z}^{M}-1} \\
\frac{\partial}{\partial I M_{z j, Z}} A_{z}^{M}\left[\sum_{z j j} \alpha_{z j j, z}^{M} I M_{z j j, z}^{-\rho_{Z}^{M}}\right]^{\frac{-1}{\rho_{Z}^{M}}}= \\
A_{Z}^{M}\left[\sum_{z j j} \alpha_{z j j, z}^{M} I M_{z j j, z}^{-\rho_{z}^{M}}\right]^{\frac{-1}{\rho_{Z}^{M}}-1} \alpha_{z j, z}^{M} I M_{z j, z}^{-\rho_{z}^{M}-1}
\end{gathered}
$$

Given (11), which is equivalent to

$$
\left(\frac{I M T_{Z}}{A_{Z}^{M}}\right)^{-\rho_{z}^{M}}=\left[\sum_{z j} \alpha_{z j, z}^{M} I M_{z j, z}^{-\rho_{Z}^{M}}\right]
$$

we can write

$$
\begin{gathered}
\frac{\partial}{\partial I M_{z j, z}} A_{Z}^{M}\left[\sum_{z j j} \alpha_{z j j, z}^{M} I M_{z j j, z}^{-\rho_{z}^{M}}\right]^{\frac{-1}{\rho_{Z}^{M}}}= \\
A_{z}^{M}\left[\left(\frac{I M T_{z}^{*}}{A_{z}^{M}}\right)^{-\rho_{z}^{M}}\right]^{\frac{-1}{\rho_{Z}^{M}}-1} \alpha_{z j, z}^{M} I M_{z j, z}^{-\rho_{Z}^{M}-1}
\end{gathered}
$$




$$
\begin{gathered}
\frac{\partial}{\partial I M_{z j, z}} A_{z}^{M}\left[\sum_{z j j} \alpha_{z j j, z}^{M} I M_{z j j, z}^{-\rho_{z}^{M}}\right]^{\frac{-1}{\rho_{z}^{M}}}= \\
A_{z}^{M}\left(\frac{I M T_{z}^{*}}{A_{z}^{M}}\right)^{\rho_{z}^{M}+1} \alpha_{z j, z}^{M} I M_{z j, z}^{-\rho_{M}^{M}-1} \\
\frac{\partial}{\partial I M_{z j, z}} A_{z}^{M}\left[\sum_{z j j} \alpha_{z j j, z}^{M} I M_{z j j, z}^{-\rho_{j}^{M}}\right]^{\frac{-1}{\rho_{z}^{M}}}= \\
\left(A_{z}^{M}\right)^{-\rho_{z}^{M}} \alpha_{z j, z}^{M}\left(\frac{I M T_{z}^{*}}{I M_{z j, z}}\right)^{\rho_{z}^{M}+1}
\end{gathered}
$$

And the first-order condition becomes

$$
\frac{\partial \mathscr{L}_{z}}{\partial I M_{z j, Z}}=P W_{z j, z}-\mu_{z}^{M}\left(A_{z}^{M}\right)^{-\rho_{z}^{M}} \alpha_{z j, z}^{M}\left(\frac{I M T_{z}^{*}}{I M_{z j, z}}\right)^{\rho_{z}^{M}+1}=0
$$

or

$$
P W_{z j, z}=\mu_{z}^{M}\left(A_{z}^{M}\right)^{-\rho_{z}^{M}} \alpha_{z j, z}^{M}\left(\frac{I M T_{z}^{*}}{I M_{z j, z}}\right)^{\rho_{z}^{M}+1}
$$

\section{F.3 Relative demand for imports of different origins}

Take the ratio of the preceding equation for two different origins, $z j$ and $z j$ :

$$
\frac{P W_{z j j, z}}{P W_{z j, z}}=\frac{\mu_{z}^{M}\left(A_{z}^{M}\right)^{-\rho_{z}^{M}} \alpha_{z j j, z}^{M}\left(\frac{I M T_{z}^{*}}{I M_{z j j, z}}\right)^{\rho_{z}^{M}+1}}{\mu_{z}^{M}\left(A_{z}^{M}\right)^{-\rho_{z}^{M}} \alpha_{z j, z}^{M}\left(\frac{I M T_{z}^{*}}{I M_{z j, z}}\right)^{\rho_{z}^{M}+1}}
$$




$$
\begin{aligned}
& \frac{P W_{z j j, z}}{P W_{z j, z}}=\frac{\alpha_{z j j, z}^{M}}{\alpha_{z j, z}^{M}}\left(\frac{I M_{z j, z}}{I M_{z j j, z}}\right)^{\rho_{z}^{M}+1} \\
& \frac{I M_{z j, z}}{I M_{z j j, z}}=\left(\frac{\alpha_{z j, z}^{M} P W_{z j j, z}}{\alpha_{z j j, z}^{M} P W_{z j, z}}\right)^{\frac{1}{\rho_{z}^{M}+1}}
\end{aligned}
$$

or, given $\rho_{Z}^{M}=\frac{1-\sigma_{Z}^{M}}{\sigma_{Z}^{M}}$ and $\rho_{Z}^{M}+1=\frac{1}{\sigma_{Z}^{M}}$,

$$
\begin{gathered}
\frac{I M_{z j, z}}{I M_{z j j, z}}=\left(\frac{\alpha_{z j, z}^{M} P W_{z j j, z}}{\alpha_{z j j, z}^{M} P W_{z j, z}}\right)^{\sigma_{z}^{M}} \\
I M_{z j, z}=\left(\frac{\alpha_{z j, z}^{M} P W_{z j j, z}}{\alpha_{z j j, z}^{M} P W_{z j, z}}\right)^{\sigma_{z}^{M}} I M_{z j j, z}
\end{gathered}
$$

F.4 Demand for components in terms of aggregate imports and prices

Substitute the last equation of F.3 into (11), and

$$
I M T_{z}^{*}=A_{z}^{M}\left\{\sum_{z j} \alpha_{z j, z}^{M}\left[\left(\frac{\alpha_{z j, z}^{M} P W_{z j j, z}}{\alpha_{z j j, z}^{M} P W_{z j, z}}\right)^{\sigma_{z}^{M}} I M_{z j j, z}\right]^{-\rho_{z}^{M}}\right\}^{\frac{-1}{\rho_{z}^{M}}}
$$

where $\rho_{Z}^{M}=\frac{1-\sigma_{z}^{M}}{\sigma_{Z}^{M}}$ implies $\sigma_{Z}^{M}=\frac{1}{\rho_{Z}^{M}+1}$. Then 
Journal of Global Economic Analysis, Volume 2 (2017), No. 1, pp. 215-324.

$$
\begin{aligned}
& I M T_{z}^{*}=A_{z}^{M}\left\{\sum_{z j} \alpha_{z j, z}^{M}\left[\left(\frac{\alpha_{z j, z}^{M} P W_{z j j, z}}{\alpha_{z j j, z}^{M} P W_{z j, z}}\right)^{\frac{1}{\rho_{z}^{M}+1}} I M_{z j j, z}\right]^{-\rho_{z}^{M}}\right\}^{\frac{-1}{\rho_{z}^{M}}} \\
& I M T_{z}^{*}=A_{z}^{M} I M_{z j j, z}\left\{\sum_{z j} \alpha_{z j, z}^{M}\left[\left(\frac{\alpha_{z j, z}^{M} P W_{z j j, z}}{\alpha_{z j j, z}^{M} P W_{z j, z}}\right)^{\frac{1}{\rho_{Z}^{M}+1}}\right]^{-\rho_{z}^{M}}\right\}^{\frac{-1}{\rho_{z}^{M}}} \\
& I M T_{z}^{*}=A_{z}^{M} I M_{z j j, z}\left[\sum_{z j} \alpha_{z j, z}^{M}\left(\frac{\alpha_{z j, z}^{M} P W_{z j j, z}}{\alpha_{z j j, z}^{M} P W_{z j, z}}\right)^{-\frac{\rho_{Z}^{M}}{\rho_{z}^{M}+1}}\right]^{\frac{-1}{\rho_{Z}^{M}}} \\
& I M T_{Z}^{*}=A_{z}^{M} I M_{z j j, z}\left(\frac{P W_{z j j, z}}{\alpha_{z j j, z}^{M}}\right)^{\frac{1}{\rho_{Z}^{M}+1}}\left[\sum_{z j} \alpha_{z j, z}^{M}\left(\frac{\alpha_{z j, z}^{M}}{P W_{z j, z}}\right)^{-\frac{\rho_{Z}^{M}}{\rho_{Z}^{M}+1}}\right]^{\frac{-1}{\rho_{Z}^{M}}}
\end{aligned}
$$




$$
\frac{I M_{z j j, z}}{I M T_{z}^{*}}=\frac{1}{A_{z}^{M}}\left(\frac{P W_{z j j, z}}{\alpha_{z j j, z}^{M}}\right)^{-\frac{1}{\rho_{z}^{M}+1}}\left[\sum_{z j} \alpha_{z j, z}^{M}\left(\frac{\alpha_{z j, z}^{M}}{P W_{z j, z}}\right)^{-\frac{\rho_{Z}^{M}}{\rho_{z}^{M}+1}}\right]^{\frac{1}{\rho_{Z}^{M}}}
$$

where $\rho_{Z}^{M}=\frac{1-\sigma_{Z}^{M}}{\sigma_{Z}^{M}}$ implies $\rho_{Z}^{M}+1=\frac{1}{\sigma_{Z}^{M}}$, and $\frac{\rho_{Z}^{M}}{\rho_{Z}^{M}+1}=1-\sigma_{Z}^{M}$. Therefore

$$
\begin{aligned}
& \frac{I M_{z j j, z}}{I M T_{z}^{*}}=\frac{1}{A_{z}^{M}}\left(\frac{P W_{z j j, z}}{\alpha_{z j j, z}^{M}}\right)^{-\sigma_{z}^{M}}\left[\sum_{z j} \alpha_{z j, z}^{M}\left(\frac{\alpha_{z j, z}^{M}}{P W_{z j, z}}\right)^{\sigma_{z}^{M}-1}\right]^{-\frac{\sigma_{z}^{M}}{\sigma_{z}^{M}-1}} \\
& \frac{I M_{z j j, z}}{I M T_{z}^{*}}=\frac{1}{A_{z}^{M}}\left(\frac{P W_{z j j, z}}{\alpha_{z j j, z}^{M}}\right)^{-\sigma_{z}^{M}}\left[\sum_{z j} P W_{z j, z}\left(\frac{\alpha_{z j, z}^{M}}{P W_{z j, z}}\right)^{\sigma_{z}^{M}}\right]^{-\frac{\sigma_{z}^{M}}{\sigma_{z}^{M}-1}}
\end{aligned}
$$

where the left-hand side ratio depends only on component prices. As a matter of fact, multiplying both sides of (F.23) by $I M T_{z}^{*}$ transforms it into a demand equation for $I M_{z j, z}$.

\section{F.5 Unit value of aggregate imports}

It is clear from (F.16) that the relative demand for imports of different origins is independent of the scale of imports, consistent with the first-degree homogeneity of aggregator function (7). Consequently, the unit value of the import aggregate can be obtained from (16) by substituting the optimal ratio determined in (F.16), and dividing through by $E_{Z} T_{z}^{*}$.

$$
P M T_{z} I M T_{z}^{*}=e_{z} I M_{z j j, z} \sum_{z j} P W_{z j, z}\left(\frac{\alpha_{z j, z}^{M} P W_{z j j, z}}{\alpha_{z j j, z}^{M} P W_{z j, z}}\right)^{\sigma_{z}^{M}}
$$


Journal of Global Economic Analysis, Volume 2 (2017), No. 1, pp. 215-324.

$$
\begin{gathered}
P M T_{z}=e_{z} \frac{I M_{z j j, z}}{I M T_{z}^{*}} \sum_{z j} P W_{z j, z}\left(\frac{\alpha_{z j, z}^{M} P W_{z j j, z}}{\alpha_{z j j, z}^{M} P W_{z j, z}}\right)^{\sigma_{z}^{M}} \\
P M T_{z}=e_{z} \frac{I M_{z j j, z}}{I M T_{z}^{*}}\left(\frac{P W_{z j j, z}}{\alpha_{z j j, z}^{M}}\right)^{\sigma_{z}^{M}} \sum_{z j} P W_{z j, z}\left(\frac{\alpha_{z j, z}^{M}}{P W_{z j, z}}\right)^{\sigma_{z}^{M}}
\end{gathered}
$$

To express $P M T_{z}$ in terms of the component prices only, we must substitute for $I M_{z j j, z} / I M T_{z}^{*}$ in the preceding equation using (F.23):

$$
\begin{gathered}
P M T_{z}=e_{z} \frac{1}{A_{z}^{M}}\left(\frac{P W_{z j j, z}}{\alpha_{z j j, z}^{M}}\right)^{-\sigma_{z}^{M}}\left[\sum_{z j} P W_{z j, z}\left(\frac{\alpha_{z j, z}^{M}}{P W_{z j, z}}\right)^{\sigma_{z}^{M}}\right]^{-\frac{\sigma_{z}^{M}}{\sigma_{z}^{M}-1}} \\
P M T_{z}=e_{z} \frac{1}{A_{z}^{M}}\left[\frac{P W_{z j j, z}}{\alpha_{z j j, z}^{M}}\right)^{\sigma_{z}^{M}} \sum_{z j} P W_{z j, z}\left(\frac{\alpha_{z j, z}^{M}}{P W_{z j, z}}\right)^{\sigma_{z}^{M}} \\
\left.\left.\left.\left.P M T_{z}=e_{z} \frac{1}{A_{z}^{M}}\left[\sum_{z j} P W_{z j, z}^{M}\right)^{\sigma_{z}^{M}}\right]^{1-\frac{\sigma_{z}^{M}}{\sigma_{z}^{M}-1}}\right]^{P W_{z j, z}}\right)^{\alpha_{z j, z}^{M}}\right]^{-\frac{1}{\sigma_{z}^{M}-1}}
\end{gathered}
$$

\section{F.6 Supply of components reformulated}

Given the preceding equation, we have 


$$
A_{z}^{M} P M T_{z}=e_{z}\left[\sum_{z j} P W_{z j, z}\left(\frac{\alpha_{z j, z}^{M}}{P W_{z j, z}}\right)^{\sigma_{z}^{M}}\right]^{-\frac{1}{\sigma_{z}^{M}-1}}
$$

Using that equation, we can rewrite (F.23) as

$$
\begin{gathered}
\frac{I M_{z j j, z}}{I M T_{z}^{*}}=\frac{1}{A_{z}^{M}}\left(\frac{P W_{z j j, z}}{\alpha_{z j j, z}^{M}}\right)^{-\sigma_{z}^{M}}\left(A_{z}^{M} P M T_{z}\right)^{\sigma_{z}^{M}} \\
\frac{I M_{z j j, z}}{I M T_{z}^{*}}=\left(\frac{1}{A_{z}^{M}}\right)^{1-\sigma_{z}^{M}}\left(\frac{P W_{z j j, z}}{\alpha_{z j j, z}^{M} P M T_{z}}\right)^{-\sigma_{z}^{M}} \\
I M_{z i j, z}=\left(\frac{1}{A_{z}^{M}}\right)^{1-\sigma_{z}^{M}}\left(\frac{P W_{z j j, z}}{\alpha_{z j j, z}^{M} P M T_{z}}\right)^{-\sigma_{z}^{M}} I M T_{z}^{*}
\end{gathered}
$$

which is an alternate form of the demand equation for imports from a particular region.

To obtain equation (B.26), substitute (D.44) from Appendix D into (F.32). 


\section{Appendix G: Equation (B.27)}

G.1 Revenue maximizing problem

The regional agent allocates exports between export destinations by maximizing the value of exports (equation (14)), subject to constraint (7) and to $\operatorname{EXT}_{Z}=E X T_{Z}^{*}$, where $E_{X T_{Z}^{*}}$ is the solution to the output allocation problem as given by equation (E.45).

G.2 Lagrangian and first-order conditions

Write the Lagrangian

$$
\mathscr{L}_{Z}=\sum_{z j} P W_{z, z j} E X_{z, z j}-\lambda_{Z}^{X}\left\{B_{Z}^{X}\left[\sum_{z j} \beta_{Z, z j}^{X}\left(E X_{Z, z j}\right)^{\kappa_{Z}^{X}}\right]^{\frac{1}{\kappa_{Z}^{X}}}-E X T_{Z}^{*}\right\}
$$

The first order conditions are:

$$
\begin{gathered}
\frac{\partial \mathscr{L}_{Z}}{\partial \lambda_{Z}^{X}}=-B_{Z}^{X}\left[\sum_{z j} \beta_{z, Z j}^{X}\left(E X_{Z, z j}\right)^{\kappa_{Z}^{X}}\right]^{\frac{1}{\kappa_{Z}^{X}}}+E X T_{Z}^{*}=0 \\
\frac{\partial \mathscr{L}_{Z}}{\partial E X_{Z, Z j}}=P W_{z, z j}-\lambda_{Z}^{X} \frac{\partial}{\partial E X_{Z, Z j}} B_{Z}^{X}\left[\sum_{z j j} \beta_{z, z j j}^{X}\left(E X_{z, z j j}\right)^{\kappa_{Z}^{X}}\right]^{\frac{1}{\kappa_{Z}^{X}}}=0
\end{gathered}
$$

where 
Journal of Global Economic Analysis, Volume 2 (2017), No. 1, pp. 215-324.

$$
\begin{aligned}
& \frac{\partial}{\partial E X_{Z, Z j}} B_{Z}^{X}\left[\sum_{z j j} \beta_{Z, Z j j}^{X}\left(E X_{Z, Z j j}\right)^{\kappa_{Z}^{X}}\right]^{\frac{1}{\kappa_{Z}^{X}}}= \\
& B_{Z}^{X} \frac{1}{\kappa_{Z}^{X}}\left[\sum_{z j j} \beta_{Z, Z j j}^{X}\left(E X_{z, z j j}\right)^{\kappa_{Z}^{X}}\right]^{\frac{1}{\kappa_{Z}^{X}}-1} \\
& \frac{\partial}{\partial E X_{z, z j}}\left[\sum_{z i j} \beta_{z, z j j}^{X}\left(E X_{z, z j j}\right)^{\kappa_{Z}^{X}}\right] \\
& \frac{\partial}{\partial E X_{Z, Z j}} B_{Z}^{X}\left[\sum_{z j j} \beta_{Z, z j j}^{X}\left(E X_{Z, z j j}\right)^{\kappa_{Z}^{X}}\right]^{\frac{1}{\kappa_{Z}^{X}}}= \\
& B_{Z}^{X} \frac{1}{\kappa_{Z}^{X}}\left[\sum_{z j j} \beta_{z, Z j j}^{X}\left(E X_{z, z j j}\right)^{\kappa_{Z}^{X}}\right]^{\frac{1}{\kappa_{Z}^{X}}-1} \beta_{z, z j}^{X} \kappa_{Z}^{X}\left(E X_{Z, z j}\right)^{\kappa_{Z}^{X}-1} \\
& \frac{\partial}{\partial E X_{z, Z j}} B_{Z}^{X}\left[\sum_{z j j} \beta_{Z, z j j}^{X}\left(E X_{Z, z j j}\right)^{\kappa_{Z}^{X}}\right]^{\frac{1}{\kappa_{Z}^{X}}}= \\
& B_{Z}^{X}\left[\sum_{z j j} \beta_{Z, z j j}^{X}\left(E X_{Z, z j j}\right)^{\kappa_{Z}^{X}}\right]^{\frac{1}{\kappa_{Z}^{X}}-1} \beta_{z, z j}^{X}\left(E X_{Z, z j}\right)^{\kappa_{Z}^{X}-1}
\end{aligned}
$$

Given (7), which is equivalent to

$$
\left(\frac{E X T_{Z}}{B_{Z}^{X}}\right)^{\kappa_{z}}=\left[\sum_{z j} \beta_{Z, Z j}^{X}\left(E X_{Z, Z j}\right)^{\kappa_{Z}^{X}}\right]
$$

we can write 


$$
\begin{gathered}
\frac{\partial}{\partial E X_{z, z j}} B_{Z}^{X}\left[\sum_{z j j} \beta_{z, z j j}^{X}\left(E X_{z, z j j}\right)^{\kappa_{Z}^{X}}\right]^{\frac{1}{\kappa_{Z}^{X}}}= \\
B_{Z}^{X}\left[\left(\frac{E X T_{Z}^{*}}{B_{Z}^{X}}\right)^{\kappa_{Z}^{X}}\right]^{\frac{1}{\kappa_{Z}^{X}-1}} \beta_{Z, z j}^{X}\left(E X_{z, z j}\right)^{\kappa_{Z}^{X}-1} \\
\frac{\partial}{\partial E X_{z, z j}} B_{Z}^{X}\left[\sum_{z j j} \beta_{z, z j j}^{X}\left(E X_{z, z j j}\right)^{\kappa_{Z}^{X}}\right]^{\frac{1}{\kappa_{Z}^{X}}}= \\
B_{Z}^{X}\left(\frac{E X T_{Z}^{*}}{B_{Z}^{X}}\right)^{1-\kappa_{Z}^{X}} \beta_{Z, z j}^{X}\left(E X_{z, z j}\right)^{\kappa_{Z}^{X}-1} \\
\frac{\partial}{\partial E X_{z, z j}} B_{Z}^{X}\left[\sum_{z i j j} \beta_{z, z j j}^{X}\left(E X_{z, z j j}\right)^{\kappa_{Z}^{X}}\right]^{\frac{1}{\kappa_{Z}^{X}}}= \\
\left(B_{Z}^{X}\right)^{\kappa_{Z}^{X}} \beta_{z, z j}^{X}\left(\frac{E X_{z, z j}}{E X T_{Z}^{*}}\right)^{\kappa_{Z}^{X}-1}
\end{gathered}
$$

And the first-order condition becomes

$$
\frac{\partial \mathscr{L}_{Z}}{\partial E X_{Z, z j}}=P W_{z, z j}-\lambda_{Z}^{X}\left(B_{Z}^{X}\right)^{\kappa_{Z}^{X}} \beta_{z, z j}^{X}\left(\frac{E X_{z, z j}}{E X T_{Z}^{*}}\right)^{\kappa_{Z}^{X}-1}=0
$$

or

$$
P W_{z, z j}=\lambda_{z}^{X}\left(B_{z}^{X}\right)^{K_{z}^{X}} \beta_{z, z j}^{X}\left(\frac{E X_{z, z j}}{E X T_{z}^{*}}\right)^{\kappa_{Z}^{X}-1}
$$




\section{G.3 Relative supply of components}

Take the ratio of the preceding equation for two different trading partners of region $z: z j$ and $z j j$.

$$
\begin{gathered}
\frac{P W_{z, z j}}{P W_{z, z j j}}=\frac{\lambda_{Z}^{X}\left(B_{Z}^{X}\right)^{\kappa_{z}^{X}} \beta_{z, z j}^{X}\left(\frac{E X_{z, z j}}{E X T_{z}^{*}}\right)^{\kappa_{Z}^{X}-1}}{\lambda_{z}^{X}\left(B_{Z}^{X}\right)^{\kappa_{z}^{X}} \beta_{z, z j j}^{X}\left(\frac{E X_{z, z j j}}{E X T_{z}^{*}}\right)^{\kappa_{Z}^{X}-1}} \\
\frac{P W_{z, z j}}{P W_{z, z j j}}=\frac{\beta_{z, z j}^{X}\left(\frac{E X_{z, z j}}{\beta_{z, z j j}^{X}}\right)^{\kappa_{Z}^{X}-1}}{\left(\frac{E X_{z, z j j}}{E X_{z, z j j}}\right)^{\kappa_{Z}^{X}-1}=\frac{\beta_{z, z j j}^{X} P W_{z, z j}}{\beta_{z, z j}^{X} P W_{z, z j j}}} \\
\frac{E X_{z, z j}}{E X_{z, z j j}}=\left(\frac{\beta_{z, z j j}^{X} P W_{z, z j}}{\beta_{z, z j}^{X} P W_{z, z j j}}\right)^{\frac{1}{\kappa_{Z}^{X}-1}}
\end{gathered}
$$

Or, given $\kappa_{Z}^{X}=\frac{\tau_{Z}^{X}+1}{\tau_{Z}^{X}}$ and $\kappa_{Z}^{X}-1=\frac{1}{\tau_{Z}^{X}}$

$$
\begin{gathered}
\frac{E X_{z, z j}}{E X_{z, z j j}}=\left(\frac{\beta_{z, z j j}^{X} P W_{z, z j}}{\beta_{z, z j}^{X} P W_{z, z j j}}\right)^{\tau_{Z}^{X}} \\
E X_{z, z j}=\left(\frac{\beta_{z, z j j}^{X} P W_{z, z j}}{\beta_{z, z j}^{X} P W_{z, z j j}}\right)^{\tau_{Z}^{X}} E X_{z, z j j}
\end{gathered}
$$

G.4 Supply of components in terms of aggregate exports and prices

Substitute the final equation of G.3 into (7) to obtain 
Journal of Global Economic Analysis, Volume 2 (2017), No. 1, pp. 215-324.

$$
E X T_{Z}^{*}=B_{Z}^{X}\left\{\sum_{z j} \beta_{z, z j}^{X}\left[\left(\frac{\beta_{z, z j j}^{X} P W_{z, z j}}{\beta_{z, z j}^{X} P W_{z, z j j}}\right)^{\tau_{Z}^{X}} E X_{z, z j j}\right]^{\kappa_{Z}^{X}}\right\}^{\frac{1}{\kappa_{Z}^{X}}}
$$

where $\kappa_{Z}^{X}=\frac{\tau_{Z}^{X}+1}{\tau_{Z}^{X}}$ implies $\tau_{Z}^{X}=\frac{1}{\kappa_{Z}^{X}-1}$, and

$$
\begin{aligned}
& E X T_{z}^{*}=B_{Z}^{X}\left\{\sum_{z j} \beta_{z, z j}^{X}\left[\left(\frac{\beta_{z, z j j}^{X} P W_{z, z j}}{\beta_{z, z j}^{X} P W_{z, z j j}}\right)^{\frac{1}{\kappa_{z}^{X}-1}} E X_{z, z j j}\right]^{\kappa_{z}^{X}}\right. \\
& E X T_{Z}^{*}=B_{Z}^{X} E X_{z, z j j}\left\{\sum_{z j} \beta_{z, z j}^{X}\left(\frac{\beta_{z, z j j}^{X} P W_{z, z j}}{\beta_{z, z j}^{X} P W_{z, z j j}}\right)^{\frac{\kappa_{Z}^{X}}{\kappa_{Z}^{X}-1}}\right\}^{\frac{1}{\kappa_{Z}^{X}}} \\
& E X T_{z}^{*}=B_{Z}^{X} E X_{z, z j j}\left(\frac{\beta_{z, z j j}^{X}}{P W_{z, z j j}}\right)^{\frac{1}{\kappa_{Z}^{X}-1}}\left\{\sum_{z j} \beta_{z, z j}^{X}\left(\frac{P W_{z, z j}}{\beta_{z, z j}^{X}}\right)^{\frac{\kappa_{z}^{X}}{\kappa_{Z}^{X}-1}}\right\}^{\frac{1}{\kappa_{Z}^{X}}}
\end{aligned}
$$




$$
\frac{E X_{z, z i j}}{E X T_{Z}^{*}}=\frac{1}{B_{Z}^{X}}\left(\frac{P W_{z, z j j}}{\beta_{z, z j j}^{X}}\right)^{\frac{1}{\kappa_{Z}^{X}-1}}\left\{\sum_{z j} \beta_{z, z j}^{X}\left(\frac{P W_{z, z j}}{\beta_{z, z j}^{X}}\right)^{\frac{\kappa_{Z}^{X}}{\kappa_{Z}^{X}-1}}\right\}^{-\frac{1}{\kappa_{z}^{X}}}
$$

where $\kappa_{Z}^{X}=\frac{\tau_{Z}^{X}+1}{\tau_{Z}^{X}}$ implies $\kappa_{Z}^{X}-1=\frac{1}{\tau_{Z}^{X}}$ and $\frac{\kappa_{Z}^{X}}{\kappa_{Z}^{X}-1}=\tau_{Z}^{X}+1$. It follows that

$$
\begin{aligned}
& \frac{E X_{z, z i j}}{E X T_{Z}^{*}}=\frac{1}{B_{Z}^{X}}\left(\frac{P W_{z, z j j}}{\beta_{z, z j j}^{X}}\right)^{\tau_{Z}^{X}}\left\{\sum_{z j} \beta_{z, z j}^{X}\left(\frac{P W_{z, z j}}{\beta_{z, z j}^{X}}\right)^{\tau_{Z}^{X}+1}\right\}^{-\frac{\tau_{X}^{X}}{\tau_{Z}^{X}+1}} \\
& \frac{E X_{z, z j j}}{E X T_{Z}^{*}}=\frac{1}{B_{Z}^{X}}\left(\frac{P W_{z, z j j}}{\beta_{z, z j j}^{X}}\right)^{\tau_{Z}^{X}}\left\{\sum_{z j} P W_{z, z j}\left(\frac{P W_{z, z j}}{\beta_{z, z j}^{X}}\right)^{\tau_{Z}^{X}}\right\}^{-\frac{\tau_{Z}^{X}}{\tau_{Z}^{X}+1}}
\end{aligned}
$$

where the left-hand side ratio depends only on component prices. As a matter of fact, multiplying both sides of (G.25) by $E X T_{z}^{*}$ transforms it into a supply equation of $E X_{z, z j}$.

\section{G.5 Unit value of aggregate exports}

It is clear from (G.18) that the relative supply on different export markets is independent of the scale of exports, consistent with the first-degree homogeneity of aggregator function (7). Consequently, the unit value of the export aggregate can be obtained from (14) by substituting the optimal ratio determined in (G.18), and dividing through by $E X T_{z}^{*}$.

$$
P X T_{z} E X T_{z}^{*}=e_{z} E X_{z, z j j} \sum_{z j} P W_{z, z j}\left(\frac{E X_{z, z j}}{E X_{z, z j j}}\right)
$$




$$
\begin{gathered}
P X T_{Z}=e_{z} \frac{E X_{z, z j j}}{E X T_{Z}^{*}} \sum_{z j} P W_{z, z j}\left(\frac{\beta_{z, z j j}^{X} P W_{z, z j}}{\beta_{z, z j}^{X} P W_{z, z j j}}\right)^{\tau_{Z}^{X}} \\
P X T_{Z}=e_{z} \frac{E X_{z, z i j}}{E X T_{z}^{*}}\left(\frac{\beta_{z, z j j}^{X}}{P W_{z, z i j}}\right)^{\tau_{Z}^{X}} \sum_{z j} P W_{z, z j}\left(\frac{P W_{z, z j}}{\beta_{z, z j}^{X}}\right)^{\tau_{Z}^{X}}
\end{gathered}
$$

To express $P X T_{z}$ in terms of the component prices only, we must substitute for $E X_{z, z j j} / E X T_{z}^{*}$ in the preceding equation using (G.25):

$$
\begin{gathered}
P X T_{Z}=e_{z} \frac{1}{B_{Z}^{X}}\left(\frac{P W_{z, z j j}}{\beta_{z, z j j}^{X}}\right)^{\tau_{Z}^{X}}\left\{\sum_{z j} P W_{z, z j}\left(\frac{P W_{z, z j}}{\beta_{z, z j}^{X}}\right)^{\tau_{Z}^{X}}\right\}^{-\frac{\tau_{Z}^{X}}{\tau_{Z}^{X}+1}} \\
\left(\frac{\beta_{z, z j j}^{X}}{P W_{z, z j j}}\right)^{\tau_{Z}^{X}} \sum_{z j} P W_{z, z j}\left(\frac{P W_{z, z j}}{\beta_{z, z j}^{X}}\right)^{\tau_{\frac{X}{Z}}^{X}} \\
P X T_{Z}=e_{z} \frac{1}{B_{Z}^{X}}\left\{\sum_{z j} P W_{z, z j}\left(\frac{P W_{z, z j}}{\beta_{z, z j}^{X}}\right)^{\tau_{Z}^{X}}\right\}^{1-\frac{\tau_{Z}^{X}}{\tau_{Z}^{X}+1}} \\
P X T_{Z}=e_{z} \frac{1}{B_{Z}^{X}}\left\{\sum_{z j} P W_{z, z j}\left(\frac{P W_{z, z j}}{\beta_{z, z j}^{X}}\right)^{\tau_{Z}^{X}}\right\}^{\frac{1}{\tau_{Z}^{X}+1}}
\end{gathered}
$$

G.6 Supply of components reformulated

Given the preceding equation, we have 


$$
B_{Z}^{X} P X T_{Z}=e_{z}\left\{\sum_{z j} P W_{z, z j}\left(\frac{P W_{z, z j}}{\beta_{z, z j}^{X}}\right)^{\tau_{Z}^{X}}\right\}^{\frac{1}{\tau_{Z}^{X}+1}}
$$

So we can rewrite (G.25) as

$$
\begin{gathered}
\frac{E X_{z, z i j}}{E X T_{Z}^{*}}=\frac{1}{B_{Z}^{X}}\left(\frac{P W_{z, z j j}}{\beta_{z, z i j}^{X}}\right)^{\tau_{Z}^{X}}\left(B_{Z}^{X} P X T_{Z}\right)^{-\tau_{Z}^{X}} \\
\frac{E X_{z, z i j}}{E X T_{Z}^{*}}=\left(\frac{1}{B_{Z}^{X}}\right)^{\tau_{Z}^{X}+1}\left(\frac{P W_{z, z j j}}{\beta_{z, z i j}^{X} P X T_{Z}}\right)^{\tau_{Z}^{X}} \\
E X_{z, z i j}=\left(\frac{1}{B_{Z}^{X}}\right)^{\tau_{Z}^{X}+1}\left(\frac{P W_{z, z i j}}{\beta_{z, z j j}^{X} P X T_{Z}}\right)^{\tau_{Z}^{X}} E X T_{Z}^{*}
\end{gathered}
$$

which is an alternate form of the supply equation of exports to a particular region.

To obtain equation (B.26), simply substitute (E.45) from Appendix E into (G.35). 


\section{Appendix H: Two-region version of Model}

In a 2-region model, each region has only one trading partner, so that, for $z j \neq z$, we have $E X T_{z}=E X_{z, z j}, I M T_{Z}=I M_{z j, z}, P W_{z j, z}=P M T_{z}$ and $P W_{z, z j}=P X T_{Z}$, which replace (7), (11), (8) and (12) respectively. The first two equalities above, together with (17), imply

$$
E X T_{z j}=I M T_{Z}
$$

And following two equalities imply

$$
P X T_{Z j}=P M T_{Z}
$$

So we can insert equations (24) and (25) into the model, and do away with equations (17) and the four equations (7), (11), (8) and (12), and with variables $E X_{z, z j}, I M_{z, z j}$ and $P W_{z, z j}$. The PWINDEX variable is consequently re-defined, and equation (20) is replaced by

$$
\text { PWINDEX }=\sqrt{\frac{\sum_{Z} P M T_{Z} I M T_{Z}^{O}}{\sum_{Z} P M T_{Z}^{O} I M T_{Z}^{O}} \frac{\sum_{Z} P M T_{Z} I M T_{Z}}{\sum_{Z} P M T_{Z}^{O} I M T_{Z}}}
$$

The two-region Model 2 variables are listed in Table H1, and the equations in Table H2. This model has $11 N+1=23$ variables and $9 N+(N-1)+1=20$ equations. There are $N+1=3$ degrees of freedom. 
Journal of Global Economic Analysis, Volume 2 (2017), No. 1, pp. 215-324.

Table H.1 Two-region Model 2 variables

\begin{tabular}{ll}
\hline Volumes & \\
\hline$Q_{Z}$ & Domestic demand for the composite good in region $z$ \\
$D_{Z}$ & Domestic demand for the locally produced good in region $z$ \\
$I M T_{Z}$ & Total imports of region $z$ \\
$E X T_{Z}$ & Total exports of region $z$ \\
$C A B X_{Z}$ & Real current account balance (pseudo-volume variable) \\
\hline Prices & \\
\hline$P_{Z}$ & Producer price \\
$P L_{Z}$ & Market price of local product \\
$P C_{Z}$ & Price of the composite good \\
$P M T_{Z}$ & Price of composite imports to region $z$ \\
$P X T_{Z}$ & Price of composite exports from region $z$ \\
$P W_{Z, z j}$ & World price of exports from region $z$ to region $z j$ \\
\hline Nominal value variables \\
\hline$C A B_{Z}$ & Current account balance of region $z$ \\
\hline
\end{tabular}


Table H.2 Two-region Model 2 equations

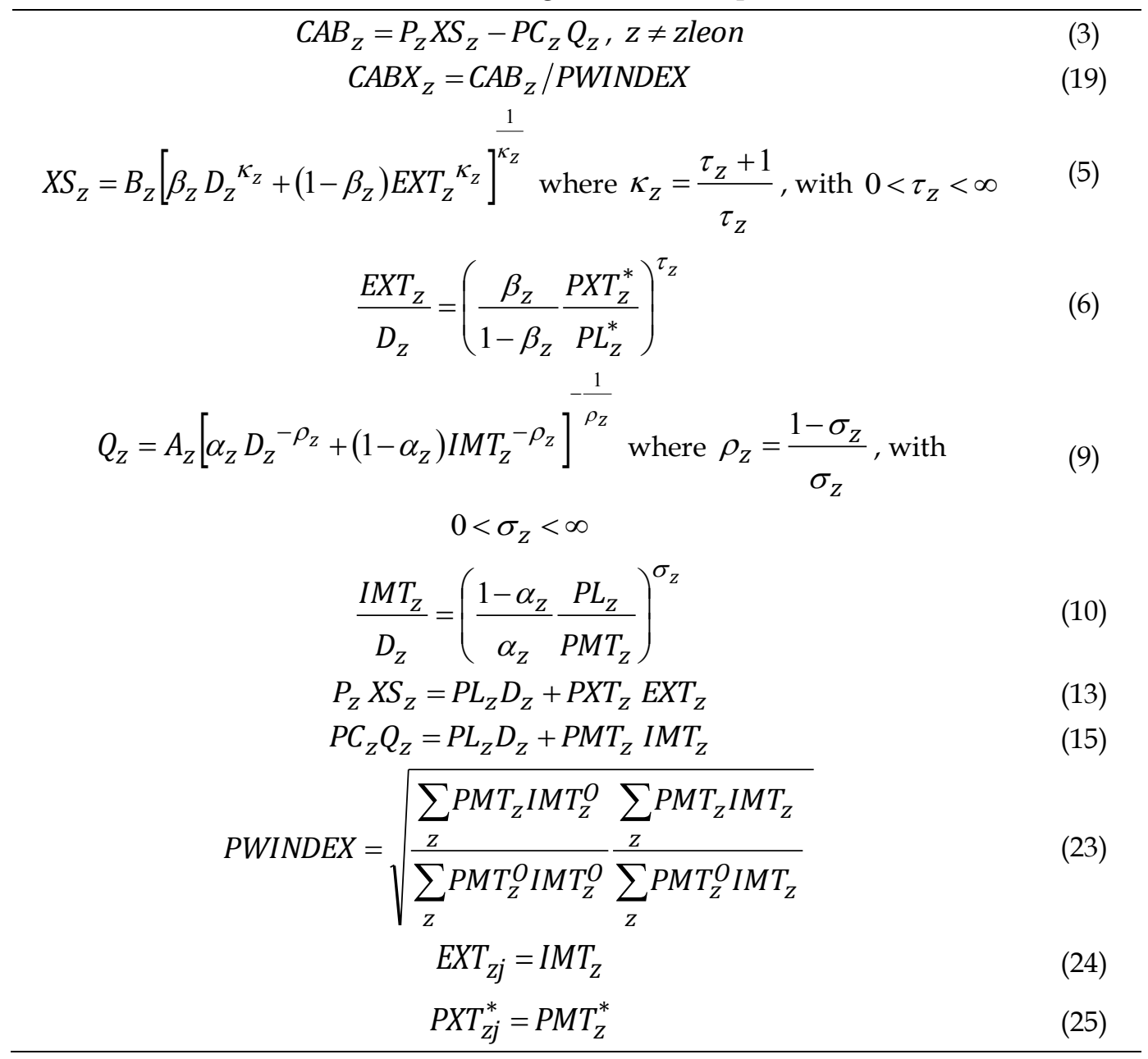




\section{Appendix I: Equation (26)}

The 2-region Q-Model consists of equations (5), (9) and (24). Develop (5) when $X S_{Z}=\overline{X S}_{Z}$

$$
\begin{aligned}
& \beta_{Z} D_{z}{ }^{\kappa_{z}}+\left(1-\beta_{z}\right) I M T_{z j}{ }^{\kappa_{z}}=\left(\frac{\overline{X S}_{z}}{B_{Z}}\right)^{\kappa_{z}} \\
& D_{Z} \kappa_{z}=\frac{1}{\beta_{Z}}\left(\frac{\overline{X S}_{Z}}{B_{Z}}\right)^{\kappa_{Z}}-\frac{\left(1-\beta_{z}\right)}{\beta_{z}} I M T_{z j} \kappa_{Z} \\
& D_{Z}=\left[\frac{1}{\beta_{Z}}\left(\frac{\overline{X S}_{Z}}{B_{Z}}\right)^{\kappa_{z}}-\frac{\left(1-\beta_{z}\right)}{\beta_{z}} I M T_{z j} \kappa^{\kappa_{z}}\right]^{\frac{1}{\kappa_{Z}}}
\end{aligned}
$$

Next, let $Q_{Z}=\bar{Q}_{Z}$, substitute into (9) and develop.

$$
\begin{aligned}
& \alpha_{Z} D_{Z}^{-\rho_{z}}+\left(1-\alpha_{Z}\right) I M T_{Z}^{-\rho_{z}}=\left(\frac{\bar{Q}_{Z}}{A_{Z}}\right)^{-\rho_{z}} \\
& D_{Z}^{-\rho_{z}}=\frac{1}{\alpha_{Z}}\left(\frac{\bar{Q}_{Z}}{A_{Z}}\right)^{-\rho_{Z}}-\frac{\left(1-\alpha_{Z}\right)}{\alpha_{Z}} I M T_{Z}^{-\rho_{Z}} \\
& D_{Z}=\left[\frac{1}{\alpha_{z}}\left(\frac{\bar{Q}_{z}}{A_{z}}\right)^{-\rho_{z}}-\frac{\left(1-\alpha_{z}\right)}{\alpha_{z}} I M T_{z}^{-\rho_{z}}\right]^{-\frac{1}{\rho_{Z}}}
\end{aligned}
$$

Combine that equation with (I.3) 
Journal of Global Economic Analysis, Volume 2 (2017), No. 1, pp. 215-324.

$$
\begin{aligned}
& {\left[\frac{1}{\alpha_{z}}\left(\frac{\bar{Q}_{z}}{A_{z}}\right)^{-\rho_{z}}-\frac{\left(1-\alpha_{z}\right)}{\alpha_{z}} I M T_{z}^{-\rho_{z}}\right]^{-\frac{1}{\rho_{z}}}=} \\
& {\left[\frac{1}{\beta_{Z}}\left(\frac{\overline{X S}_{Z}}{B_{Z}}\right)^{\kappa_{z}}-\frac{\left(1-\beta_{Z}\right)}{\beta_{Z}} I M T_{z j} \kappa_{z}\right]^{\frac{1}{\kappa_{Z}}}} \\
& \frac{1}{\alpha_{Z}}\left(\frac{\bar{Q}_{Z}}{A_{Z}}\right)^{-\rho_{z}}-\frac{\left(1-\alpha_{Z}\right)}{\alpha_{Z}} I M T_{Z}^{-\rho_{z}}= \\
& {\left[\frac{1}{\beta_{Z}}\left(\frac{\overline{X S}_{Z}}{B_{Z}}\right)^{\kappa_{z}}-\frac{\left(1-\beta_{z}\right)}{\beta_{z}} I M T_{z j} \kappa_{z}\right]^{-\frac{\rho_{Z}}{\kappa_{z}}}} \\
& \frac{\left(1-\alpha_{z}\right)}{\alpha_{z}} I M T_{Z}^{-\rho_{z}}= \\
& \frac{1}{\alpha_{Z}}\left(\frac{\bar{Q}_{Z}}{A_{Z}}\right)^{-\rho_{z}}-\left[\frac{1}{\beta_{Z}}\left(\frac{\overline{X S}_{Z}}{B_{Z}}\right)^{\kappa_{z}}-\frac{\left(1-\beta_{Z}\right)}{\beta_{z}} I M T_{z j} \kappa_{z}\right]^{-\frac{\rho_{Z}}{\kappa_{z}}} \\
& I M T_{Z}^{-\rho_{z}}=\frac{1}{\left(1-\alpha_{z}\right)}\left(\frac{\bar{Q}_{Z}}{A_{z}}\right)^{-\rho_{z}} \\
& -\frac{\alpha_{z}}{\left(1-\alpha_{z}\right)}\left[\frac{1}{\beta_{z}}\left(\frac{\overline{X S}_{Z}}{B_{Z}}\right)^{\kappa_{z}}-\frac{\left(1-\beta_{z}\right)}{\beta_{z}} I M T_{z j}{ }^{\kappa_{z}}\right]^{-\frac{\rho_{Z}}{\kappa_{z}}}
\end{aligned}
$$

Equation (26) follows directly. 


\section{Appendix J: Equation (27)}

The 2-region P-Model consists of equations (6), (10) and (25). Substitute from (25) into (6) and develop.

$$
\begin{gathered}
\frac{\beta_{z}}{1-\beta_{z}} \frac{P M T_{z j}}{P L_{z}}=\left(\frac{E X T_{z}}{D_{z}}\right)^{1 / \tau_{z}} \\
\frac{1-\beta_{z}}{\beta_{z}} \frac{P L_{z}}{P M T_{z j}}=\left(\frac{D_{z}}{E X T_{z}}\right)^{1 / \tau_{z}} \\
P L_{z}=\frac{\beta_{z}}{1-\beta_{z}}\left(\frac{D_{z}}{E X T_{z}}\right)^{1 / \tau_{z}} P M T_{z j}
\end{gathered}
$$

Next, develop (10):

$$
\begin{gathered}
\frac{1-\alpha_{z}}{\alpha_{z}} \frac{P L_{z}}{P M T_{z}}=\left(\frac{I M T_{z}}{D_{z}}\right)^{1 / \sigma_{z}} \\
P L_{z}=\frac{\alpha_{z}}{1-\alpha_{z}}\left(\frac{I M T_{z}}{D_{z}}\right)^{1 / \sigma_{z}} P M T_{Z}
\end{gathered}
$$

Combine this with (J.3):

$$
\begin{aligned}
& \frac{\alpha_{Z}}{1-\alpha_{Z}}\left(\frac{I M T_{Z}}{D_{Z}}\right)^{1 / \sigma_{Z}} P M T_{Z}=\frac{\beta_{Z}}{1-\beta_{Z}}\left(\frac{D_{Z}}{E X T_{Z}}\right)^{1 / \tau_{Z}} P M T_{z j} \\
& P M T_{Z}=\frac{1-\alpha_{Z}}{\alpha_{Z}}\left(\frac{D_{Z}}{I M T_{Z}}\right)^{1 / \sigma_{z}} \frac{\beta_{Z}}{1-\beta_{Z}}\left(\frac{D_{Z}}{E X T_{Z}}\right)^{1 / \tau_{z}} P M T_{Z j}
\end{aligned}
$$

Equation (27) follows directly. 


\section{Appendix K: GAMS implementation}

\section{K.1 Brief description of GAMS programs}

Model 3 has been implemented in GAMS for illustration purposes. All of the GAMS programs and files are provided with this paper as supplementary online material. The standard version of the model is implemented in program SkeletonWorld_2017_Model3A.gms. The main program calls several sub-programs. In order of appearance, they are:

- Calib_check_2017.gms may be called at the end of calibration. It computes the difference between the left- and right-hand side of every equation when the variable arguments are replaced by their (calibrated) benchmark values. Since this is a toy model, the Calib_check_2017 results are displayed in the main program listing file (SkeletonWorld_2017_Model3A.lst).

- Closures_2017.gms contains various closure options, including different choices of the numéraire and of its value. The user chooses by activating/disactivating the \$ontext/\$offtext switches in the program.

- RESULTS_BAU_2017.gms stores the BAU (“business as usual”, no shock) solution values.

- Benchmk_chk_2017.gms may be called once the BAU solution has been computed. It computes the difference between each variable's solution value and its benchmark (calibrated) value. Since this is a toy model, the Benchmk_chk_2017 results are displayed in the main program listing file (SkeletonWorld_2017_Model3A.lst).

- RESULTS_SIM_2017.gms stores the SIM solution values and produces the GDX output file and its xls companion.

- RATIOS_2017.gms computes the ratio of SIM solution values to BAU (benchmark) values for the purpose of checking for calibration consistency.

Calib_check_2017.gms and Benchmk_chk_2017.gms are not directly related to the issues discussed in this paper. They are two diagnostic tools frequently used by the author in developing models.

The program SkeletonWorld_2017_Model3A.gms produces two result files. The first is a "classic" Excel results file (results.xls $x$ ) created in RESULTS_SIM_2017.gms from the standard GDX output file, using GDX2XLS, with one sheet per variable. The second (Results_too.xlsx) is in tabular form, made in the main program with the GDXXRW facility, reading set-up parameters from a text file which is created within the GAMS model program.

There is a second program, SkeletonWorld_2017_Model3B.gms, which is basically the same, but offers in addition various possibilities for modifying the 
calibration and/or the closure rules, and making comparisons with the standard version of the model.

Some of the sub-programs called by SkeletonWorld_2017_Model3A.gms are duplicated to be called by SkeletonWorld_2017_Model3B.gms.

- Closures_B_2017.gms is similar to Closures_2017.gms, except for the user's choice of which closure is activated; it also offers additional closures designed to perform tests of Model A against Model B. If the user chooses the same closure in both, then SkeletonWorld_2017_Model3A.gms and_Model3B.gms will produce identical results. Duplicating the Closures program eliminates the need to modify the choice of closures between SkeletonWorld_2017_Model3A.gms and_Model3B.gms.

- In RESULTS_BAU_B_2017.gms, variables containing the BAU ("business as usual", no shock) solution values are prefixed with " $\mathrm{B}$ " for comparison computations. For instance, the variable $\operatorname{BvalDD}(\mathrm{z}, \mathrm{scen})$ corresponds to valDD(z,scen) created in RESULTS_BAU_2017.gms.

- RESULTS_SIM_B_2017.gms stores the SIM solution values with a B prefix like the preceding profram, and produces the GDX output file and its xls companion.

- RATIOS_B_2017.gms computes the ratio of SIM solution values to BAU (benchmark) values for the purpose of checking for calibration consistency. The ratio variables names begin with a B for comparison computations. For example, the variable BratioEXT(z) corresponds to ratioEXT(z) created in RATIOS_2017.gms.

Comparisons are made using two sub-programs:

- Compare_SOLUTIONS.gms reads the standard model solution GDX file produced by SkeletonWorld_2017_Model3A.gms, and computes the ratios of Model3B/Model3A solution values, for the purpose of checking for model homogeneity when the choice of the numeraire, or its value, or both are modified. The output consists of files Sol_ratios.gdx and Sol_ratios.xls.

- Compare_RATIOS.gms reads the GDX file of SIM/BAU ratios produced by SkeletonWorld_2017_Model3A.gms, and computes the Model3BModel3A ratio differences, for the purpose of checking for calibration neutrality and consistency. The output consists of files Ratio_diff.gdx and Ratio_diff.xls.

SkeletonWorld_2017_Model3B.gms is therefore a tool for testing model homogeneity and calibration neutrality and consistency. 


\section{K.2 Examples of tests}

All the tests described here have been made against the standard version of the model with the FP closure and PWINDEX as the numéraire price:

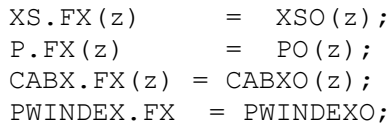

In Model3B, the closure is defined in Closures_B_2017.gms, as described for each test.

\section{K.2.1 Test of FP and FE closures}

Implement the FE closure in Model3B:

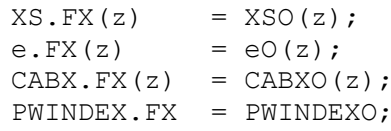

In Compare_SOLUTIONS.gms, set the Lambda parameter to 1 and the program will compute the ratios of Model3B/Model3A solution values, after dividing all regional prices and nominal variables by their exchange rate to convert them into the international currency. Observe in Sol_ratios.xls that all ratios are equal to 1 (except for exchange rates): the two models are identical.

\section{K.2.2 Homogeneity test 1}

If a model is truly homogenous, the solution values of real (volume) variables and all price and nominal value ratios are supposed to be

- independent of which commodity is taken as the numéraire;

- independent of which region is taken as the reference region when the numéraire is a regional commodity (a particular case of the preceding);

- independent of the particular value given the price of the numéraire, whatever commodity plays that role.

In Model3B, implement the FP closure with $P W_{z r, z r j}$ as the numéraire.

- In section 1.1 of SkeletonWorld_2017_Model3B.gms, designate a pair of reference regions:

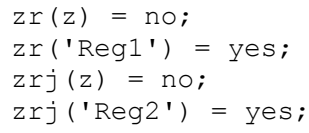

(this may also be done in Closures_B_2017.gms)

- In Closures_B_2017.gms, activate "Homogeneity test 1 with FP closure":

$$
\begin{array}{ll}
X S \cdot F X(z) & X S O(z) ; \\
P \cdot F X(z) & =
\end{array}
$$




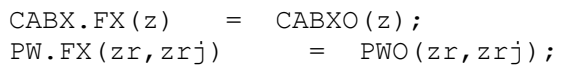

In Compare_SOLUTIONS.gms, set the $\lambda$ parameter using 29

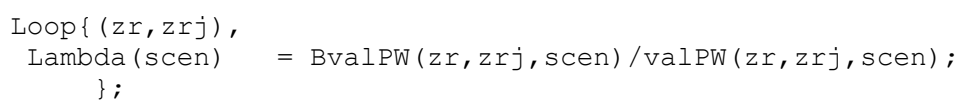

Then the program will compute the ratios of Model3B/Model3A solution values, after dividing all regional prices and nominal variables by their exchange rate to convert them into the international currency. Observe in Sol_ratios.xls that all ratios are equal to 1 (except for exchange rates): the two models are identical. As for exchange rates, their ratio is equal to $1 / \lambda$. Now, let $P L_{Z}^{A}$ be the value of $P L_{Z}$ in the Model3A solution, and $P L_{Z}^{B}$ its value in the Model3B solution. We observe in Sol_ratios.xls that $P L_{z}^{B} / e_{z}^{B}=\lambda P L_{Z}^{A} / e_{z}^{A}$ and $e_{z}^{B} / e_{z}^{A}=1 / \lambda$. Consequently, $P L_{Z}^{B} / P L_{Z}^{A}=1$, which is as it should be under the FP closure. The reason is that the regional numéraires (here $P_{Z}$ ) are fixed, so that, if the models are identical, going from $P W I N D E X$ to $P W_{z r, z r j}$ as the numéraire leaves regional prices unchanged.

Homogeneity test 1 can be performed with the same results if the numéraire is given any arbitrary positive value. For example,

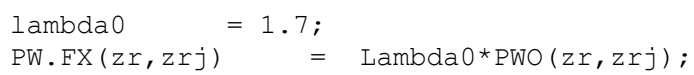

However, with multiples outside the $[0.45,1.7]$ range, the model needs to be initialized accordingly for GAMS to be able to solve it:

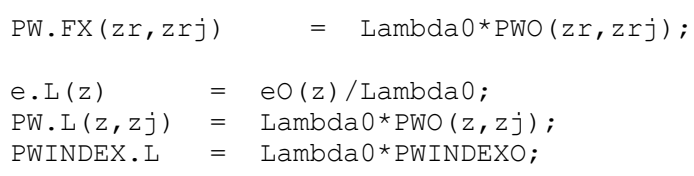

(the re-initialization code appears in Closures_B_2017.gms).

\section{K.2.3 Homogeneity test 2}

In Model3B, implement the FP closure with $e_{z r}$ as the numéraire. Activate "Homogeneity test 2 with FP closure" in Closures_B_2017.gms:

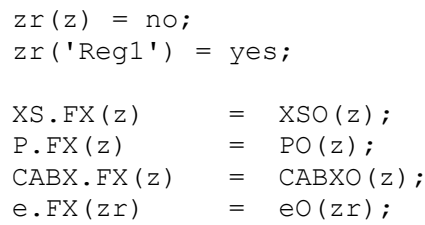

${ }^{29}$ The loop is necessary because $z r$ and $z r j$ are sets (albeit singletons) in GAMS. 
In Compare_SOLUTIONS.gms, set the $\lambda$ parameter using ${ }^{30}$

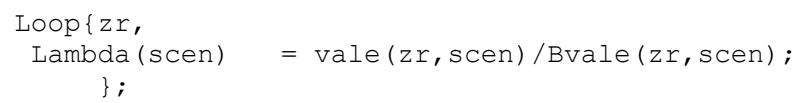

which is equivalent to $\lambda=\left(1 / e_{Z r}^{B}\right) /\left(1 / e_{z r}^{A}\right)$. This reflects the fact that the numéraire is an international price: it is actually $1 / e_{z r}$, the price of region $z r^{\prime}$ s currency in terms of the international currency, rather than $e_{z r}$, the price of the international currency in terms of region $z r^{\prime}$ s currency.

The program will compute the ratios of Model3B/Model3A solution values, after dividing all regional prices and nominal variables by their exchange rate to convert them into the international currency. Observe in Sol_ratios.xls that all ratios are equal to 1 (except for exchange rates): the two models are identical.

Homogeneity test 2 can be performed with the same results if the numéraire is given any arbitrary positive value, but with large or small multiples, the model needs to be initialized accordingly for GAMS to be able to solve it:

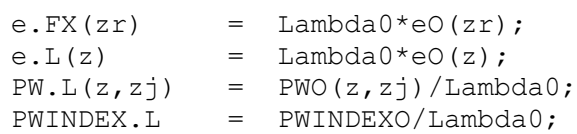

The appropriate code is in Closures_B_2017.gms.

\section{K.2.4 Homogeneity test 3}

In Model3B, implement the FP closure with PWINDEX as the numéraire and change $P_{Z}$ for $P L_{z}$ as the regional numéraire. Activate "Homogeneity test 3 with FP closure: change in regional numeraire" in Closures_B_2017.gms:

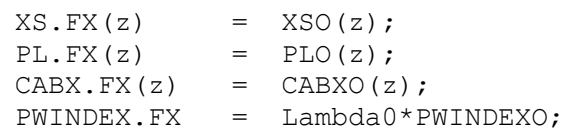

and add the corresponding re-initialization:

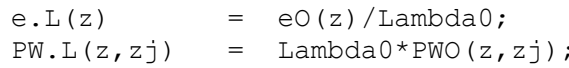

In Compare_SOLUTIONS.gms, set the $\lambda$ parameter using

Lambda (scen) = BvalPWINDEX (scen)/valPWINDEX (scen);

The program will compute the ratios of Model3B/Model3A solution values, after dividing all regional prices and nominal variables by their exchange rate to convert them into the international currency. Observe in Sol_ratios.xls that all ratios are equal to 1 (except for exchange rates): the two models are identical.

\footnotetext{
30 The loop is necessary because $z r$ and $z r j$ are sets (albeit singletons) in GAMS.
} 


\section{K.2.5 Homogeneity test 4}

In Model3B, implement the FP closure with PWINDEX as the numéraire and change $P_{z}$ for $P L_{z}$ as the regional numéraire, with a change of value for the numéraire. Activate "Homogeneity test 4 with FP closure: change in regional numeraire" in Closures_B_2017.gms:

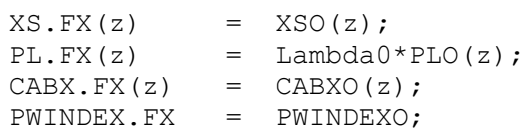

and add the corresponding re-initialization:

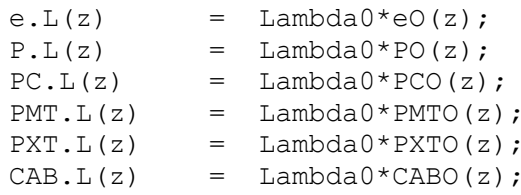

In Compare_SOLUTIONS.gms, set the $\lambda$ parameter using

Lambda (scen) = BvalPWINDEX (scen)/ValPWINDEX (scen);

The program will compute the ratios of Model3B/Model3A solution values, after dividing all regional prices and nominal variables by their exchange rate to convert them into the international currency. Observe in Sol_ratios.xls that all ratios are equal to 1 (except for exchange rates): the two models are identical.

\section{K.2.6 Homogeneity test 5 with FE closure}

The modified FE closure is implemented in Model3B with $P_{z r}$ rather than PWINDEX as numéraire. Activate "Homogeneity test 5 with FE closure and regional price for numéraire" in Closures_B_2017.gms:

$$
\begin{array}{ll}
\mathrm{XS} \cdot \mathrm{FX}(z) & \mathrm{XSO}(\mathrm{z}) ; \\
\mathrm{e} \cdot \mathrm{FX}(\mathrm{z}) & =\mathrm{eO}(\mathrm{z}) ; \\
\mathrm{CABX} \cdot \mathrm{FX}(\mathrm{z}) & =\mathrm{CABXO}(\mathrm{z}) ; \\
\mathrm{P} \cdot \mathrm{FX}(\mathrm{zr}) & =\operatorname{Lambda0}{ }^{\mathrm{PO}}(\mathrm{zr}) ;
\end{array}
$$

and add the corresponding re-initialization:

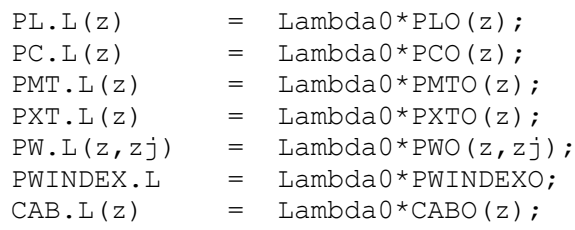

In Compare_SOLUTIONS.gms, set the $\lambda$ parameter using

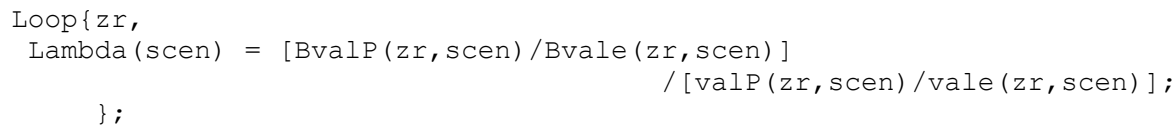


The program will compute the ratios of Model3B/Model3A solution values, after dividing all regional prices and nominal variables by their exchange rate to convert them into the international currency. Observe in Sol_ratios.xls that all ratios are equal to 1 (except for exchange rates): the two models are identical.

\section{K.2.7 Calibration consistency test of price $\times$ volume factoring}

In Closures_B_2017.gms, implement the standard FP closure for Model3B with PWINDEX as the numéraire. Then, to perform the test, go to section 4.1 of the SkeletonWorld_2017_Model3B.gms main program, look for the line

*\$GoTo Alternate_calib

and remove the asterisk at the beginning. In Compare_SOLUTIONS.gms, set the $\lambda$ parameter using

Lambda (scen) = 1;

The program will compute the ratios of Model3B/Model3A solution values, after dividing all regional prices and nominal variables by their exchange rate to convert them into the international currency. Observe in Sol_ratios.xls that the ratios of the prices of aggregates $\left(P X T_{Z}, P M T_{Z}, P C_{Z}\right.$ and $\left.P_{Z}\right)$ are equal to the Model3B/Model3A ratios of their benchmark values, while the ratios of the corresponding volumes are the inverse, including in the SIM results. In Ratio_diff.xls, observe that the SIM/BAU ratios are equal in both models, which satisfies the criterion of calibration consistency as stated in equations (1) and (2) of section 2.3.31

\section{K.2.8 Calibration consistency test of arbitrary prices}

Implement the standard FP closure in Model3B with PWINDEX as the numéraire.

In section 3.4 of the SkeletonWorld_2017_Model3B.gms program, replace the following statements

$$
\begin{array}{ll}
\mathrm{eO}(z) & =1 ; \\
\operatorname{PLO}(z) & =1 ; \\
\operatorname{PWO}(z, z j) & =1 ;
\end{array}
$$

with different assignments. For example:

$$
\begin{array}{ll}
\mathrm{eO}(z) & =.5 ; \\
\mathrm{PLO}(z) & =0.8 ; \\
\mathrm{PWO}(z, z j) & =1.5 ; \\
\mathrm{PLO}\left(\operatorname{Reg}^{\prime}\right) & =1.6 ; \\
\mathrm{PWO}(\mathrm{zr}, \mathrm{zrj}) & =2 ;
\end{array}
$$

${ }^{31}$ Actually, there are very small differences, due to imperfect accuracy in the calculations. 
In Sol_ratios.xls, the SIM ratios and the BAU ratios are equal, and the passionate reader could trace the sources of divergence from 1. More interesting is Ratio_diff.xls, where it can be verified that the SIM/BAU ratios are equal in both models, which satisfies the criterion of calibration consistency as stated in section 1.3 .32

\section{K.2.9 Calibration consistency test of arbitrary prices and exchange rates}

Implement the standard FP closure in Model3B with PWINDEX as the numéraire. In the SkeletonWorld_2017_Model3B.gms program, go to the section labeled "4.1_supplement Re-calibration with arbitrary unequal exchange rates", and activate the procedure by cancelling the \$ontext/\$offtext switches. The same result obtains as in the preceding test.

32 Actually, there are very small differences, due to imperfect accuracy in the calculations. 